\title{
ON CONFORMAL MAPPING OF INFINITE STRIPS
}

BY

\author{
S. E. WARSCHAWSKI
}

\section{INTRODUCTION}

Let $S$ be the strip in the plane of the complex variable $w=u+i v$ defined by the relations

$$
\phi_{-}(u)<v<\phi_{+}(u), \quad-\infty<u<+\infty,
$$

where $\phi_{-}(u), \phi_{+}(u)$ are continuous for $-\infty<u<+\infty$. Let $\theta(u) \equiv \phi_{+}(u)-\phi_{-}(u)$ and $\psi(u) \equiv \frac{1}{2}\left[\phi_{+}(u)+\phi_{-}(u)\right]$. $S$ can be mapped conformally onto the strip $|y|<\pi / 2$ of the $z$-plane, $z=x+i y$, by means of an analytic function $z=Z(w)=X(w)+i Y(w)$ in such a manner that $\lim _{u \rightarrow+\infty} X(w)=+\infty$. The principal object of this paper is to obtain asymptotic expressions for $Z(w)$ and its derivative $Z^{\prime}(w)$ as $u \rightarrow+\infty$. For this purpose two inequalities concerning the difference $X\left(w_{2}\right)-X\left(w_{1}\right)\left(w_{1}=u_{1}+i v_{1}, w_{2}=u_{2}+i v_{2}\right.$ in $\left.S\right)$ are established which are similar to certain inequalities of Ahlfors $\left({ }^{1}\right)$, but which, due to some assumptions regarding the smoothness of the boundary of $S$, yield sharper estimates for large values of $u_{1}$ and $u_{2}$.

We say that $S$ is an $L$-strip $\left({ }^{2}\right)$ with the boundary inclination $\gamma$ at $u=+\infty$, $|\gamma|<\pi / 2$, if, for $u_{2}>u_{1}$,

$$
\frac{\phi_{+}\left(u_{2}\right)-\phi_{+}\left(u_{1}\right)}{u_{2}-u_{1}}, \quad \frac{\phi_{-}\left(u_{2}\right)-\phi_{-}\left(u_{1}\right)}{u_{2}-u_{1}}
$$

approach the same limit, $\tan \gamma$, as $u_{1}$ and $u_{2} \rightarrow+\infty$ simultaneously. The two inequalities in question (the "basic inequalities") are then as follows:

I. If $S$ is an L-strip with the boundary inclination $\gamma=0$ at $u=+\infty$, then

$$
X\left(w_{2}\right)-X\left(w_{1}\right) \leqq \pi \int_{u_{1}}^{u_{2}} \frac{1+\psi^{\prime 2}(u)}{\theta(u)} d u+\frac{\pi}{12} \int_{u_{1}}^{u_{2}} \frac{\theta^{\prime 2}(u)}{\theta(u)} d u+o(1),
$$

where $o(1) \rightarrow 0$ as $u_{1}, u_{2} \rightarrow+\infty$, uniformly. with respect to $v_{1}$ and $v_{2}$.

II. If $S$ is an L-strip as in I and if, in addition, $\phi_{+}^{\prime}(u)$ and $\phi_{-}^{\prime}(u)$ are continuous and of bounded variation for $u_{0} \leqq u \leqq+\infty$, then

$$
X\left(w_{2}\right)-X\left(w_{1}\right) \geqq \pi \int_{u_{1}}^{u_{2}} \frac{1+\psi^{\prime 2}(u)}{\theta(u)} d u-\frac{\pi}{4} \int_{u_{1}}^{u_{2}} \frac{\theta^{\prime 2}(u)}{\theta(u)} d u+o(1) .
$$

Presented to the Society, January 1, 1941; received by the editors January 23, 1941.

(1) Ahlfors [1, p. 10 and p. 16]. The number in the brackets refers to the author's paper quoted in the bibliography.

(2) For a justification of this notation see $\$ 1(b)$. 
If now the integral $\int_{u_{0}}^{\infty}\left[\theta^{\prime 2}(u) / \theta(u)\right] d u$ converges, I and II together yield an asymptotic expression for the difference $X\left(w_{2}\right)-X\left(w_{1}\right)$ :

$$
X\left(w_{2}\right)-X\left(w_{1}\right)=\pi \int_{u_{1}}^{u_{2}} \frac{1+\psi^{\prime 2}(u)}{\theta(u)} d u+o(1) \quad \text { as } u_{1}, u_{2} \rightarrow+\infty .
$$

Combining this with a result on $Y(w)$ established in this paper under the same hypothesis as in I, we obtain the following asymptotic representation for $Z(w)$ :

$$
Z(w)=\lambda+\pi \int_{u_{0}}^{u} \frac{1+\psi^{\prime 2}(t)}{\theta(t)} d t+i \pi \frac{v-\psi(u)}{\theta(u)}+o(1), \quad \text { as } u \rightarrow+\infty,
$$

uniformly with respect to $v$. Here $\lambda$ is a real constant.

As to $Z^{\prime}(w)$, we find under the same hypothesis as in I,

$$
Z^{\prime}(w) \sim \frac{\pi}{\theta(u)} \quad \text { as } u \rightarrow+\infty,
$$

uniformly in any subregion $S_{\beta}:\{(|v-\psi(u)|) / \theta(u) \leqq \beta / \pi\}$ where $0<\beta<\pi / 2$. The "approach of $u$ to $+\infty$ in any $S_{\beta}$ " is the analogue of the "approach within any angle" in the case of a finite boundary point at which the boundary curve possesses a tangent. In this connection we obtain an extension of Carathéodory's well known theorem which states that the map of a region bounded by a Jordan curve onto a circle is quasi-conformal at a boundary point which is the vertex of a corner (cf. $\$ 16)$.

Similar expressions for $Z(w)$ and $Z^{\prime}(w)$ are obtained when $\gamma \neq 0$, but $|\gamma|<\pi / 2$. Further theorems are derived as corollaries from the above mentioned results.

An important part in the proof of some of these results is played by a theorem of A. Qstrowski (cf. §2) which deals with the argument of the derivative of the mapping function in a neighborhood of a point at which the boundary curve has a cusp. In the present paper a new proof of this theorem is given.

By the use of suitable transformations these results can be applied to the study of the mapping function in a neighborhood of a boundary point for various boundary configurations. Let $\Gamma$ be a closed Jordan curve in the $\omega$-plane, $R$ the interior of $\Gamma$, let $\zeta=\zeta(\omega)$ map $R$ conformally onto the circle $|\zeta-1|<1$, let $\omega_{0}$ be a point on $\Gamma$ and let $\zeta\left(\omega_{0}\right)=0$. By means of simple logarithmic transformations, asymptotic expressions for $\zeta(\omega)$ and $\zeta^{\prime}(\omega)$ as $\omega \rightarrow \omega_{0}$ are derived from the above stated results. In particular, when $\Gamma$ possesses a tangent at $\omega_{0}$, the expression for $\zeta(\omega)$ yields a new criterion for the existence of the derivative of $\zeta(\omega)$ at $\omega_{0}$ (i.e., $\lim _{\omega \rightarrow \omega_{0}} \zeta(\omega) /\left(\omega-\omega_{0}\right)$ for unrestricted approach). Another case is that in which $\Gamma$ has a cusp at $\omega_{0}$. It was partly for the purpose of treating this case that the present investigation was started. 
In addition to the asymptotic expressions for $\zeta(\omega)$ and $\zeta^{\prime}(\omega)$ as $\omega \rightarrow \omega_{0}$ we obtain some extensions of earlier results on the cusp which are due to Ostrowski.

Other applications include the study of $\zeta(\omega)$ for a region $R$ whose boundary contains two "concurrent" spirals. These spirals may both approach a single "asymptotic" point $\omega_{0}$, or else the set of their limiting points might be a more general point set which forms a prime end of $R$.

\section{Preliminaries}

1. Definitions. We begin with a few definitions which will be used in the paper.

(a) Simple Jordan strip. Let $C_{+}$and $C_{-}$be two curves in the w-plane $(w=u+i v)$ represented by continuous functions

$$
v=\phi_{+}(u), \quad v=\phi_{-}(u), \quad u \geqq u_{0}, \quad \phi_{+}(u)>\phi_{-}(u),
$$

respectively, and let $C_{1}$ be a Jordan arc $\left(^{3}\right)$ which lies in the half-plane $u \leqq u_{0}$ and connects the finite end points of $C_{+}$and $C_{-}$. The curve $C$, consisting of $C_{+}, C_{-}$ and $C_{1}$ decomposes the complex plane into two regions (by Jordan's theorem). Let $S$ be the one which contains the region

$$
\phi_{-}(u)<v<\phi_{+}(u), \quad u_{0}<u<+\infty .
$$

We call $S$ a simple Jordan strip.

We set $\theta(u)=\phi_{+}(u)-\phi_{-}(u)$ and denote by $\theta_{u}$ the segment $\{R w=u$, $\left.\phi_{-}(u) \leqq v \leqq \phi_{+}(u)\right\}$.

(b) $L$-TANGENT AT $u=+\infty$; $L$-STRIP. Let $C$ be a curve represented by the equation $v=\phi(u)$ where $\phi(u)$ is continuous for $u_{0} \leqq u<+\infty$. We say that $C$ has an L-tangent with the angle of inclination $\gamma,-\pi / 2 \leqq \gamma \leqq \pi / 2$ at $u=+\infty$, if the angle of inclination of any chord $w_{1} w_{2}$ of $C\left(w_{1}=u_{1}+i v_{1}, w_{2}=u_{2}+i v_{2}\right), u_{1}<u_{2}$, approaches the limit $\gamma$ when $u_{1}$ and $u_{2}$ approach $+\infty$ simultaneously, or, in other words, if for every $\epsilon>0$ there exists an $R(\epsilon)>0$ such that for the principal value of the argument,

$$
\left|\arg \left(w_{2}-w_{1}\right)-\gamma\right|<\epsilon \quad \text { when } u_{2}>u_{1}>R(\epsilon) .
$$

Let $S$ be a simple Jordan strip whose boundary curves $C_{+}$and $C_{-}$have both $L$-tangents at $u=+\infty$ with the angles of inclination $\gamma_{+}$and $\gamma_{-},\left|\gamma_{+}\right|<\pi / 2$, $\left|\gamma_{-}\right|<\pi / 2$, respectively. Then, for sufficiently large $u$, say $u \geqq u_{1} \geqq u_{0}, \theta(u)$ has bounded difference quotients and hence $\theta^{\prime}(u)$ exists for $u \geqq u_{1}$, except possibly for a set of measure zero. Furthermore, $\theta^{\prime}(u)$ is bounded and $\theta(b)-\theta(c)=\int_{c}^{b} \theta^{\prime}(u) d u$, $b \geqq c \geqq u_{1}$.

If, in particular, the L-tangents of $C_{+}$and $C_{-}$at $u=+\infty$ have the same angle of inclination $\gamma$, then we call $S$ an L-strip with the boundary inclination $\gamma$ at $u=+\infty$.

( $\left.{ }^{3}\right) C_{1}$ may pass through the infinite point ( $u=-\infty$ "). 
The definition of an $L$-tangent at $u=+\infty$ is patterned after that of an $L$-tangent to a curve at a finite point $\left.{ }^{4}\right)$. Let $\beta$ be a Jordan arc which possesses a tangent at one of its end points, $P$. If the angle of inclination of every chord $P_{1} P_{2}$ of $\beta\left(P_{1} \neq P_{2}\right)$, approaches that of the tangent at $P$ as $P_{1}$ and $P_{2}$ approach $P$ simultaneously, then we say that $\beta$ has an $L$-tangent at $P$.

2. A theorem of Ostrowski on $L$-cusps. Let $\Gamma$ be a closed Jordan curve. If, in a neighborhood of a point $P$ of $\Gamma, \Gamma$ consists of two branches $\Gamma_{+}$and $\Gamma_{-}$ each of which possesses a tangent at $P$, and if the interior angle made by these two tangents is $\theta, 0<\theta \leqq 2 \pi$, then we shall say that $\Gamma$ has a corner of measure $\theta$ at $P$. The limiting case where $\theta=0$ will be called a cusp. If both tangents at $P$ are $L$-tangents then we shall say that $\Gamma$ has an $L$-corner $(\theta>0)$ or an $L$-cusp $(\theta=0)$ at $P$.

The following theorem is due to A. Ostrowski( $\left.{ }^{5}\right)$.

TheOREM I. (Ostrowski.) Let $\Gamma$ be a closed Jordan curve in the $\omega$-plane which has an L-cusp at the point $P$ of $\Gamma$, and let $\omega=f(\zeta)$ map the circle $|\zeta|<1$ conformally onto the interior of $\Gamma$ in such a manner that $\zeta=1$ corresponds to $P$. Then, for any determination of the argument in $|\zeta|<1, \lim _{\zeta \rightarrow 1} \arg \left[f^{\prime}(\zeta)(\zeta-1)\right]$ exists for unrestricted approach.

This theorem is an extension of a theorem by Lindelöf $\left({ }^{6}\right)$ on $\arg f^{\prime}(\zeta)$ in the case that $\Gamma$ has an $L$-corner of measure $\theta=\pi$ at $P$, and was first stated and proved by Ostrowski $\left({ }^{5}\right)$. We give here a proof of this theorem, which is different from the one by Ostrowski. It follows to some extent the ideas which Lindelöf used in proving his theorem.

Proof of Theorem I. (i) It may be assumed that $P$ is the point $\omega=0$, that $\Gamma_{-}$follows $\Gamma_{+}$when $\Gamma$ is traversed in the mathematically positive direction and that the $L$-tangents to $\Gamma_{+}$and $\Gamma_{-}$at $\omega=0$ fall on the negative real axis. Then we have, for a proper choice of the determination of the argument for $\omega_{1}$ and $\omega_{2}$ on $\Gamma_{-}$,

$$
\lim _{\omega_{1} \omega_{2} \rightarrow 0} \arg \left(\omega_{2}-\omega_{1}\right)=\pi \quad\left(\omega_{1} \text { between } \omega=0 \text { and } \omega_{2}\right)
$$

and for $\omega_{1}$ and $\omega_{2}$ on $\Gamma_{+}$,

$$
\lim _{\omega_{1}, \omega_{2} \rightarrow 0} \arg \left(\omega_{2}-\omega_{1}\right)=2 \pi \quad\left(\omega_{2} \text { between } \omega_{1} \text { and } \omega=0\right) .
$$

Let $\gamma_{+}$and $\gamma_{-}$be subarcs of $\Gamma_{+}$and $\Gamma_{-}$, respectively, such that both have $\omega=0$ as an end point and that for all $\omega_{1}, \omega_{2}$ on $\gamma_{-}$

$$
\left|\arg \left(\omega_{2}-\omega_{1}\right)-\pi\right|<\pi / 8 \quad\left(\omega_{1} \text { between } \omega_{2} \text { and } \omega=0\right)
$$

(4) The idea of an $L$-tangent at a finite point was introduced by Lindelöf, [1, pp. 89-91], the term " $L$-tangent" by Ostrowski $[1$, p. 93$]$.

(5) Ostrowski [1, pp. 181-183].

(6) Lindelöf [1, pp. 89-91]. 
and for all $\omega_{1}, \omega_{2}$ on $\gamma_{+}$,

$$
\left|\arg \left(\omega_{2}-\omega_{1}\right)-2 \pi\right|<\pi / 8 \quad\left(\omega_{2} \text { between } \omega=0 \text { and } \omega_{1}\right) .
$$

It follows from (2.3) and (2.4) that $\boldsymbol{\gamma}_{-}$and $\gamma_{+}$can be represented in the form $(\omega=\xi+i \eta)$ :

$$
\eta=g_{-}(\xi), \quad \eta=g_{+}(\xi),
$$

respectively. Both of these functions are continuous and have derivatives almost everywhere in a certain interval $\xi_{0} \leqq \xi \leqq 0\left(\xi_{0}<0\right)$. We can assume both functions have a derivative at $\xi_{0}$, so that $\gamma_{-}$and $\gamma_{+}$have tangents at the points $P_{1}\left(\xi_{0}, g_{-}\left(\xi_{0}\right)\right)$ and $P_{2}\left(\xi_{0}, g_{+}\left(\xi_{0}\right)\right)$, respectively. Denote the arcs $O P_{1}$ and $O P_{2}$ by $\Gamma_{-}^{\prime}$ and $\Gamma_{+}^{\prime}$, respectively.

Let $\gamma$ be an arc with continuously turning tangent which joins $P_{1}$ and $P_{2}$, which has the same tangents at $P_{1}$ and $P_{2}$ as $\Gamma$, and which lies in the interior $R$ of $\Gamma$ except for its end points. Call $\Gamma_{1}$ the closed Jordan curve formed by $\gamma$, $\Gamma_{-}^{\prime}$ and $\Gamma_{+}{ }^{\prime}$. We shall prove the theorem first for the function $\omega=f_{1}(\zeta)$ which maps the circle $|\zeta|<1$ onto the interior $R_{1}$ of $\Gamma_{1}$, and for which $f_{1}(1)=0$.

(ii) We establish first an auxiliary inequality. Let a be a point on $\Gamma_{+}^{\prime}$ and $b$ a point on $\Gamma_{-}$( so that $R a \geqq \xi_{0}, R b \geqq \xi_{0}$ ). If that determination of the argument is chosen which lies between $-\pi / 2$ and $3 \pi / 2$ (inclusive),

$$
-\frac{\pi}{8} \leqq \arg (b-a) \leqq \pi+\frac{\pi}{8} .
$$

Assume first that $R b>R a$. Then only the left-hand side of (2.5) needs proof, since then $\arg (b-a) \leqq \pi / 2$. Draw the line $\xi=R a$. This line intersects $\Gamma_{-} \prime$ in a point $a^{\prime}$ whose ordinate is greater than that of $a$. By use of elementary properties of the angles of a triangle, it is easily seen that

$$
\arg (b-a) \geqq \arg \left(b-a^{\prime}\right), \quad \arg \left(b-a^{\prime}\right) \geqq-\pi / 8,
$$

because of (2.3), since $b$ and $a^{\prime}$ are both on $\Gamma_{-}^{\prime}$ and $b$ is between $a^{\prime}$ and 0 . The case $R b<R a$ is treated similarly. If $R a=R b, \arg (b-a)=\pi / 2$ and (2.5) is true.

(iii) Let $f_{1}\left(e^{i \theta_{1}}\right)$ and $f_{1}\left(e^{i \theta_{2}}\right)$ be interior points of $\Gamma_{-}^{\prime}$ and $\Gamma_{+}^{\prime}$, respectively, $0<\theta_{1}<\theta_{2}<2 \pi$, and let $\delta_{0}$ be a positive number which is less than $\min \left\{\theta_{1}, 2 \pi-\theta_{2}\right\}$ and also so small that (2.3) and (2.4) are satisfied on the arcs of $\Gamma_{1}$ corresponding to $0 \leqq \theta \leqq \theta_{1}+\delta_{0}$ and $\theta_{2}-\delta_{0} \leqq \theta \leqq 2 \pi$ of $|\zeta|=1$, respectively. Since $\omega=f_{1}(\zeta)$ is continuous and univalent on $|\zeta|=1$,

$$
Q(\theta ; \delta) \equiv \arg \left[f_{1}\left(e^{i(\theta+\delta)}\right)-f_{1}\left(e^{i \theta}\right)\right], \quad 0<\delta \leqq \delta_{0}, \delta \text { fixed },
$$

is continuous for all real $\theta$, once the determination for one value, say $\theta=0$, is selected. Since $(2.3)$ holds on $\Gamma_{-}, Q(\theta ; \delta)$ can be determined by the condition $|Q(0 ; \delta)-\pi|<\pi / 8$. We have then also

$$
|Q(\theta ; \delta)-\pi|<\pi / 8 \quad \text { for } 0 \leqq \theta \leqq \theta_{1} .
$$


We assert now that

$$
|Q(\theta ; \delta)-2 \pi|<\pi / 8 \quad \text { for } \theta_{2}-\delta \leqq \theta \leqq 2 \pi-\delta .
$$

To prove this it is sufficient to show that

$$
\left|Q\left(\theta_{2} ; \delta\right)-2 \pi\right|<\pi / 8 \text {. }
$$

For since (2.4) holds on the arc of $\Gamma_{1}$ which corresponds to thearc $\theta_{2}-\delta_{0} \leqq \theta \leqq 2 \pi$ of $|\zeta|=1$ and $Q(\theta ; \delta)$ is continuous in $\theta,(2.7)$ will then follow.

To prove (2.8) we observe first that for any $\theta$,

$$
Q(\theta+2 \pi ; \delta)-Q(\theta ; \delta)=2 \pi \text {. }
$$

This is an application of the principle of the argument, since the function $f_{1}\left(\zeta e^{i \delta}\right)-f_{1}(\zeta)$ is regular in $|\zeta|<1$, continuous in $|\zeta| \leqq 1$, and has exactly one zero, $\zeta=0$, in $|\zeta| \leqq 1$.

Now let $Q\left(\theta_{2} ; \delta\right)=h_{2}+2 k \pi$ where $k$ is an integer and $\left|h_{2}\right|<\pi / 8$ (because of (2.4)). Since $Q(\theta ; \delta)$ is continuous, it follows from (2.4) and (2.5) that for $\theta_{2} \leqq \theta \leqq 2 \pi$

$$
2 k \pi-\pi / 8 \leqq Q(\theta ; \delta) \leqq 2 k \pi+\pi+\pi / 8 .
$$

Let $Q(0 ; \delta)=h_{1}+\pi,\left|h_{1}\right|<\pi / 8$ by (2.6). Then by (2.9)

$$
Q(2 \pi ; \delta)=h_{1}+3 \pi
$$

and hence by (2.10) for $\theta=2 \pi$

$$
2 k \pi-\pi / 8 \leqq h_{1}+3 \pi \leqq 2 k \pi+\pi+\pi / 8 \text {. }
$$

The left-hand side of this inequality implies $k \leqq 1$, the right-hand side $k \geqq 1$, so that $k=1$ and (2.8) is proved.

If we set $\omega_{1}=f_{1}\left(e^{i \theta}\right), \omega_{2}=f_{1}\left(e^{i(\theta+\delta)}\right)$ then $(2.6)$ shows that $Q(\theta ; \delta)$ for $0 \leqq \theta \leqq \theta_{1}$ is identical with the determination of arg $\left(\omega_{2}-\omega_{1}\right)$ chosen in (2.1). Hence, by (2.1), $Q(\theta ; \delta) \rightarrow \pi$ as $\theta$ and $\delta$ approach 0 simultaneously. Similarly, we infer from (2.7) and (2.2) that $Q(\theta ; \delta) \rightarrow 2 \pi$ as $\theta \rightarrow 2 \pi$ and $\delta \rightarrow 0$ with the restriction that $\theta \leqq 2 \pi-\delta$.

Finally we note that there exists an $M>0$ such that for all $0 \leqq \theta \leqq 2 \pi$ and all $0<\delta \leqq \delta_{0}$

$$
|Q(\theta ; \delta)| \leqq M \text {. }
$$

For $0 \leqq \theta \leqq \theta_{1}$ this follows from (2.6) for $\theta_{2}-\delta \leqq \theta \leqq 2 \pi-\delta$ from (2.7), for $2 \pi-\delta \leqq \theta \leqq 2 \pi$ from (2.10) with $k=1$, and for $\theta_{1} \leqq \theta \leqq \theta_{2}-\delta$ from (2.3), (2.4) and the fact that $\gamma$ has a continuously turning tangent.

(iv) For fixed $\delta \leqq \delta_{0}$, the function

$$
g(\zeta ; \delta) \equiv \frac{f_{1}\left(\zeta e^{i \delta}\right)-f_{1}(\zeta)}{\zeta\left(e^{i \delta}-1\right)} \text { for } \zeta \neq 0, g(0 ; \delta)=f_{1}^{\prime}(0),
$$


is regular in $|\zeta|<1$, continuous in $|\zeta| \leqq 1$, and never vanishes because $f_{1}(\zeta)$ is univalent in $|\zeta| \leqq 1$. Therefore, any branch of $\arg g(\zeta ; \delta)$ is harmonic in $|\zeta|<1$ and continuous in $|\zeta| \leqq 1$. We choose that branch of $\arg g(\zeta ; \delta)$ which reduces to

$$
Q(\theta ; \delta)-\delta / 2-\theta-\pi / 2, \quad 0 \leqq \theta \leqq 2 \pi,
$$

for $|\zeta|=1$. Consider now, for $|\zeta|<1$, the harmonic function

$$
P(\zeta ; \delta)=\arg [g(\zeta ; \delta)(\zeta-1)]=\arg g(\zeta ; \delta)+\arg (\zeta-1),
$$

where $\arg (\zeta-1)$ is determined by the condition that it reduces to $\pi$ for $\zeta=0$. $P(\zeta ; \delta)$ is bounded in $|\zeta|<1$ and it has continuous boundary values on $|\zeta|=1$ except at $\zeta=1$. Hence it can be represented by the Poisson integral

$$
P(\zeta ; \delta)=\frac{1}{2 \pi} \int_{0}^{2 \pi} P\left(e^{i \theta} ; \delta\right) \frac{\left(1-\rho^{2}\right) d \theta}{1+\rho^{2}-2 \rho \cos (\theta-\alpha)}, \quad \zeta=\rho e^{i \alpha}, \quad \rho<1 .
$$

(v) Let $\epsilon>0$ be given. Then by our statement at the end of section (iii) there exists an $\eta=\eta(\epsilon)<\epsilon / 4$ such that, for all positive $\delta \leqq \min \left(\delta_{0}, \eta\right)$ :

$$
|Q(\theta ; \delta)-\pi|<\epsilon / 4 \quad \text { for } 0 \leqq \theta \leqq \eta
$$

and

$$
|Q(\theta ; \delta)-2 \pi|<\epsilon / 4 \quad \text { for } 2 \pi-\eta \leqq \theta \leqq 2 \pi-\delta .
$$

Observing that, for the determination of $\arg (\zeta-1)$ selected in (2.12)

$$
\arg \left(e^{i \theta}-1\right)=\frac{\theta}{2}+\frac{\pi}{2}, \quad 0<\theta<2 \pi,
$$

we find, for $0<\theta<\eta$,

$$
\begin{aligned}
\left|P\left(e^{i \theta} ; \delta\right)-\pi\right| & =\left|Q(\theta ; \delta)-\frac{\delta}{2}-\theta-\frac{\pi}{2}+\left(\frac{\theta}{2}+\frac{\pi}{2}\right)-\pi\right| \\
& \leqq|Q(\theta ; \delta)-\pi|+\frac{\delta}{2}+\frac{|\theta|}{2} \\
& \leqq|Q(\theta ; \delta)-\pi|+\frac{\delta}{2}+\frac{\eta}{2},
\end{aligned}
$$

and for $2 \pi-\eta \leqq \theta \leqq 2 \pi-\delta$,

$$
\begin{aligned}
\left|P\left(e^{i \theta} ; \delta\right)-\pi\right| & \leqq|Q(\theta ; \delta)-2 \pi|+\frac{\delta}{2}+\left|\frac{\theta}{2}-\pi\right| \\
& \leqq|Q(\theta ; \delta)-2 \pi|+\frac{\delta}{2}+\frac{\eta}{2} .
\end{aligned}
$$

Because of (2.13) and (2.14), we have therefore 


$$
\begin{aligned}
\left|P\left(e^{i \theta} ; \delta\right)-\pi\right| & <\frac{\epsilon}{4}+\frac{\epsilon}{8}+\frac{\epsilon}{8}=\frac{\epsilon}{2} \\
& \text { for } 0 \leqq \theta \leqq \eta \text { and } 2 \pi-\eta \leqq \theta \leqq 2 \pi-\delta .
\end{aligned}
$$

To estimate the difference $|P(\zeta ; \delta)-\pi|$ we decompose the Poisson integral as follows:

$$
P(\zeta ; \delta)=\frac{1}{2 \pi}\left\{\int_{0}^{\eta}+\int_{\eta}^{2 \pi-\eta}+\int_{2 \pi-\eta}^{2 \pi-\delta}+\int_{2 \pi-\delta}^{2 \pi}\right\} .
$$

Then we find, from (2.15), (2.12), and (2.11),

$$
\begin{aligned}
|P(\zeta ; \delta)-\pi| \leqq & \frac{1}{2 \pi} \frac{\epsilon}{2} \int_{0}^{\eta} \frac{\left(1-\rho^{2}\right) d \theta}{1+\rho^{2}-2 \rho \cos (\theta-\alpha)} \\
& +\frac{M+2 \pi}{2 \pi} \frac{2 \pi\left(1-\rho^{2}\right)}{1+\rho^{2}-2 \rho \cos (\eta-|\alpha|)} \\
& +\frac{1}{2 \pi} \frac{\epsilon}{2} \int_{2 \pi-\eta}^{2 \pi-\delta} \frac{\left(1-\rho^{2}\right) d \theta}{1+\rho^{2}-2 \rho \cos (\theta-\alpha)} \\
& +\frac{M+2 \pi}{2 \pi} \cdot \frac{\delta\left(1-\rho^{2}\right)}{(1-\rho)^{2}} .
\end{aligned}
$$

Now we keep $\zeta$ fixed and let $\delta \rightarrow 0$. Since $M$ is independent of $\delta$, we find

$$
\left|\arg \left[f_{1}^{\prime}(\zeta)(\zeta-1)\right]-\pi\right| \leqq \epsilon+(M+2 \pi) \frac{1-\rho^{2}}{1+\rho^{2}-2 \rho \cos (\eta-|\alpha|)} .
$$

Hence, as $\zeta \rightarrow 1$,

$$
\lim \sup \left|\arg \left[f_{1}^{\prime}(\zeta)(\zeta-1)\right]-\pi\right| \leqq \epsilon,
$$

q.e.d.

(vi) It remains to prove the theorem for the original function $f(\zeta)$. The inverse function of $\omega=f(\zeta)$ maps $R_{1}$ onto a region $E$ of the circle $|\zeta|<1$ and the arc $P_{1} O P_{2}$ of $\Gamma_{1}$ onto an arc of the circumference $|\zeta|=1$ which contains $\zeta=1$ as an interior point. If $\zeta=\zeta(s), \zeta(1)=1$, is a suitably chosen function which maps the circle $|s|<1$ onto $E$, then $f_{1}(s)=f(\zeta(s))$. By Schwarz's reflection principle, $\zeta(s)$ is regular at $\zeta=1$ and $\zeta^{\prime}(1) \neq 0$. Now we have, in a sufficiently small neighborhood of $\zeta=1,|\zeta|<1$,

$$
\arg f^{\prime}(\zeta)=\arg f_{1}^{\prime}(s)-\arg \zeta^{\prime}(s)
$$

and

$$
\arg \left[f^{\prime}(\zeta)(\zeta-1)\right]=\arg \left[f_{1}^{\prime}(s)(s-1)\right]-\arg \zeta^{\prime}(s)+\arg \frac{\zeta-1}{s-1}
$$


Letting $\zeta \rightarrow 1$ in $|\zeta|<1$ and consequently $s \rightarrow 1$ in $|s|<1$, we find the desired result from (2.16).

3. An application. As an application of Theorem I we prove the following

THEOREM II. Let $S$ be an L-strip with the boundary inclination $\gamma=0$ at $u=+\infty$. Let $w=W(z), z=x+i y$, map the strip $|y|<\pi / 2$ conformally onto $S$ in such a manner that $R W(x+i y) \rightarrow+\infty$ as $x \rightarrow+\infty$. Then

(a) For a suitable choice of the branch of the argument, $\lim _{x \rightarrow+\infty} \arg W^{\prime}(z)=0$, for unrestricted approach.

(b) For $z_{1}$ and $z_{2}$ in any fixed strip $|y| \leqq \beta<\pi / 2$ which satisfy the condition $\left|z_{2}-z_{1}\right| \leqq M$ (M a constant), $\lim \left[W^{\prime}\left(z_{2}\right) / W^{\prime}\left(z_{1}\right)\right]=1$ as $x_{1}=R z_{1}$ (and hence $\left.x_{2}=R z_{2}\right)$ approaches $+\infty$, uniformly in $|y| \leqq \beta\left({ }^{7}\right)$.

Proof. (a) The transformation $\omega=1 / w$ maps $S$ onto a region $R$ bounded by a closed Jordan curve $\left.{ }^{8}\right) \Gamma$ in such a manner that $u=+\infty$ corresponds to $\omega=0$. Let $w_{1}$ and $w_{2}$ be two points on one of the boundary curves $C_{+}$or $C_{-}$of $S$ and $\omega_{1}=1 / w_{1}$ and $\omega_{2}=1 / w_{2}$ their images on $\Gamma$. Since $S$ is an $L$-strip with the boundary inclination $\gamma=0$ at $u=+\infty$, the principal value of arg $w$ $(-\pi<\arg w \leqq \pi)$ is single-valued in $S$ if $u=R w$ is sufficiently large, and arg $w_{1}$ and arg $w_{2}$ approach 0 as $w_{1}$ and $w_{2}$ approach $\infty$ along $C_{+}$or $C_{-}$. Hence, it follows from the relation $\arg \left(\omega_{2}-\omega_{1}\right)=\arg \left(w_{1}-w_{2}\right)-\arg w_{1}-\arg w_{2}$, which holds when the principal value is taken for each of the arguments, provided $R w_{1}>R w_{2}$ and both are sufficiently large, that $\Gamma$ has an $L$-cusp at $\omega=0$.

Let now, for $|\zeta|<1, z=\log [(1+\zeta) /(1-\zeta)]$ be the branch of the logarithm which is 0 when $\zeta=0$. The function

$$
\omega=f(\zeta)=\frac{1}{W(z)}, \quad z=\log \frac{1+\zeta}{1-\zeta}
$$

maps the circle $|\zeta|<1$ conformally onto $R$. It can be defined as a continuous function in $|\zeta| \leqq 1$, and then $f(1)=0$. Hence by Theorem I,

$$
\lim _{\zeta \rightarrow 1} \arg \left[f^{\prime}(\zeta)(\zeta-1)\right]=l
$$

exists, or

$$
\lim _{x \rightarrow+\infty} \arg W^{\prime}(z)=\lim _{\zeta \rightarrow 1} \arg \left[(W(z))^{2} f^{\prime}(\zeta) \frac{\zeta^{2}-1}{2}\right]=l
$$

exists. That this limit is 0 for a suitable choice of the branch of the argument can be seen in the following way. The image $L$ in the $w$-plane of the real axis

${ }^{(7)}$ Part (b) is due to Ostrowski [1, p. 185, relation (68.6), and p. 177, relation (64.2)].

(8) If the arc $C_{1}$ of the boundary curve of $S$ passes through $w=\infty, \Gamma$ will have a double point at $\omega=0$. In this case, however, $\Gamma$ will be a Jordan curve on the Riemann surface of $(\omega-a)^{1 / 2}$ for a suitable choice of the point $a(a \neq 0)$. 
in the z-plane by means of $w=W(z)$ has a tangent at the point $W(x)$ which forms the angle arg $W^{\prime}(x)$ with the positive $u$-axis. If $l \neq 0$, we may assume that $l$ is also not a multiple of $2 \pi$. Since $L$ lies in $S$, the slope of any chord through two points $W\left(x_{0}\right)$ and $W\left(x_{1}\right), x_{0} f i x e d$, will approach 0 as $x_{1} \rightarrow+\infty$, no matter how large $x_{0}$ might be chosen. On the other hand, $\lim _{x \rightarrow+\infty} \arg W^{\prime}(z)=l$ implies that $L$ has an $L$-tangent with the direction $l$ at $u=+\infty$, which is impossible unless $l=0$ or a multiple of $2 \pi$.

(b) To prove part (b) we note first: If $F(z)=U(z)+i V(z)$ is regular for $|y|<\pi / 2$ and if $\lim _{x \rightarrow+\infty} V(z)=V_{0}$ in $|y|<\pi / 2$, then uniformly in any fixed sub-strip $|y| \leqq \beta<\pi / 2, \lim _{x \rightarrow+\infty} F^{\prime}(z)=0\left({ }^{9}\right)$.

This statement follows immediately from the integral representation $\left({ }^{10}\right)$ :

$$
F^{\prime}(z)=\frac{i}{\pi r} \int_{0}^{2 \pi} V\left(z+r e^{i \theta}\right) e^{-i \theta} d \theta=\frac{i}{\pi r} \int_{0}^{2 \pi}\left[V\left(z+r e^{i \theta}\right)-V_{0}\right] e^{-i \theta} d \theta,
$$

where $0<r<\pi / 2-\beta, z=x+i y,|y| \leqq \beta$.

Now, if $z_{1}$ and $z_{2}$ are points in $|y| \leqq \beta<\pi / 2$, and $\left|z_{2}-z_{1}\right| \leqq M$,

$$
\left|F\left(z_{2}\right)-F\left(z_{1}\right)\right|=\left|\int_{z_{1}}^{z_{2}} F^{\prime}(\zeta) d \zeta\right|=o\left(\left|z_{2}-z_{1}\right|\right)=o(1)
$$

as $x_{1} \rightarrow \infty$, uniformly in $|y| \leqq \beta$. Applying this result to

$$
F(z) \equiv \log W^{\prime}(z)=\log \left|W^{\prime}(z)\right|+i \arg W^{\prime}(z)
$$

and using the result of part (a), we have, for $\left|z_{2}-z_{1}\right| \leqq M$,

or

$$
\lim _{x_{1} \rightarrow+\infty}\left[\log W^{\prime}\left(z_{2}\right)-\log W^{\prime}\left(z_{1}\right)\right]=0
$$

$$
\lim _{x_{1} \rightarrow+\infty} \frac{W^{\prime}\left(z_{2}\right)}{W^{\prime}\left(z_{1}\right)}=1
$$

q.e.d.

4. Some auxiliary results. As an application of Theorem II we prove the following

LeMma 1. Let $S$ be an L-strip in the w-plane with the boundary inclination 0 at $u=+\infty$. For all $u$ exceeding a certain number $u_{1}$, let $l_{u}$ denote a line segment within $S$ which joins the point $w=u+i \phi_{-}(u)$ of $C_{-}$with some point of $C_{+}$and which forms the angle $\gamma(u), \lim _{u \rightarrow+\infty} \gamma(u)=0$, with the positive v-axis $\left({ }^{11}\right)$. Let.

(9) This result is well known; see for example, Wolff $[1$, p. 221, §6], Ostrowski [2, p. 23, Theorem V, Part 3]. For the last part of this proof compare Ostrowski [2, p. 31, Theorem VII].

(10) Copson [1, p. 88, Example 1].

(11) The angle $\gamma$ which a line forms with the positive $v$-axis is the smaller of the two angles between them (if there be a smaller one), and it is considered as positive if the direction of rotation from the positive $v$-axis to the line is counterclockwise. 
$z=Z(w)=X(w)+i Y(w)$ map the strip $S$ conformally onto the strip $|y|<\pi / 2$ in such a manner that $\lim _{u \rightarrow+\infty} X(w)=+\infty$. If

$$
x^{*}=\max _{w \in l_{u}} X(w), \quad x_{*}=\min _{w \in l_{u}} X(w), \quad m(u)=\max _{w \in l_{u}}\left|\arg Z^{\prime}(w)\right|,
$$

then, for all sufficiently large $u$,

$$
x^{*}-x_{*} \leqq 2 \pi \tan \{m(u)+|\gamma(u)|\} \rightarrow 0 \quad \text { as } u \rightarrow+\infty .
$$

Proof. By Theorem I, a suitable branch of $\arg Z^{\prime}(w)$ approaches 0 uniformly in $S$ as $u \rightarrow+\infty$. Let, for $u \geqq u_{2} \geqq u_{1},\left|\arg Z^{\prime}(w)\right|<\pi / 8,|\gamma(u)|<\pi / 8$. The image of $l_{u}$ in the $z$-plane by means of $z=Z(w)$, is an arc $\lambda_{u}$ joining $z^{*}=Z\left(u+i \phi_{-}(u)\right)$ on $y=-\pi / 2$ with a point on $y=\pi / 2$. If $w \in l_{u}$ (w in the interior of $S)$, then $\lambda_{u}$ has a tangent at $z=Z(w)$ which forms the angle $\tau=\arg Z^{\prime}(w)+\gamma(u)$ with the positive $y$-axis. A simple application of the mean value theorem shows that no point of $\lambda_{u}$ is in the exterior of the isosceles triangle whose top is at $z^{*}$, whose base is on $y=\pi / 2$ and whose angle at $z^{*}$ is $2[m(u)+|\gamma(u)|]$. The base of this triangle is $2 \pi \tan [m(u)+|\gamma(u)|]$, and therefore $x^{*}-x_{*} \leqq 2 \pi \tan [m(u)+|\gamma(u)|]$.

Theorem III. Let $S$ be a simple Jordan strip and let $z=Z(w)=X(w)+i Y(w)$ map $S$ conformally onto the strip $|y|<\pi / 2$ in such a manner that $\lim _{u \rightarrow+\infty} X(w)$ $=+\infty$. Let $w_{1}=u_{1}+i v_{1}, w_{2}=u_{2}+i v_{2}, u_{0} \leqq u_{1} \leqq u_{2}, x_{1}=X\left(w_{1}\right), x_{2}=X\left(w_{2}\right)$. Then

(a) the integral

$$
\pi \int_{u_{1}}^{u_{2}} \frac{d u}{\theta(u)} \leqq x_{2}-x_{1}+4 \pi ;
$$

(b) if $S$ is an L-strip with the boundary inclination 0 at $u=+\infty$,

$$
\pi \int_{u_{1}}^{u_{2}} \frac{d u}{\theta(u)} \leqq x_{2}-x_{1}+o(1) \quad \text { as } u \rightarrow+\infty,
$$

uniformly with respect to $v_{1}, v_{2}$.

Proof. (a) Part (a) is a theorem of Ahlfors (see Ahlfors [1, p. 10] or Nevanlinna [1, p. 92]).

(b) In the proof of (4.1) an inequality (Ahlfors [1, p. 8, relation (3) or Nevanlinna [1, p. 90, relation (35)]) is first derived which contains the following

$$
\pi \int_{u_{1}}^{u_{2}} \frac{d u}{\theta(u)} \leqq x_{2}^{*}-x_{* 1},
$$

where $x_{* 1}=\min _{w \in \theta_{u}} X(w), x_{2}^{*}=\max _{w \in \theta_{u}} X(w)$. Now, by Lemma 1, (applied with $\left.l_{u} \equiv \theta_{u}\right), x_{2}^{*}-x_{2} \rightarrow 0$ and $x_{1}-x_{*_{1} \rightarrow 0} \rightarrow$ as $u_{1} \rightarrow+\infty$. Hence, if we write $x_{2}^{*}=x_{2}+\left(x_{2}^{*}-x_{2}\right), x_{* 1}=x_{1}+\left(x_{* 1}-x_{1}\right)$, the result (4.2) follows immediately. 


\section{The FIRST BASIC INEQUALITY}

5. Statement of Theorem IV. The content of Theorem IV (b) will be referred to as the first basic inequality.

THEOREM IV. Let $S$ be a simple Jordan strip and let the functions $v=\phi_{+}(u)$ and $v=\phi_{-}(u)$ representing the boundary curves $C_{+}$and $C_{-}$of $S$ have uniformly bounded difference quotients for $u \geqq u_{0}$. Let

$$
\theta(u)=\phi_{+}(u)-\phi_{-}(u), \quad \psi(u)=\frac{1}{2}\left[\phi_{+}(u)+\phi_{-}(u)\right] .
$$

Suppose that the function $w=W(z)=U(z)+i V(z), z=x+i y$, maps the strip $|y|<\pi / 2$ conformally onto $S$ in such a manner that $x=+\infty$ corresponds to $u=+\infty$. Let $z_{1}=x_{1}+i y_{1}$ and $z_{2}=x_{2}+i y_{2}$ be two points in $|y|<\pi / 2, x_{1}<x_{2}$, and let $u_{1}=U\left(z_{1}\right), u_{2}=U\left(z_{2}\right)$.

(a) If $\left|\phi_{+}^{\prime}(u)\right|$ and $\left|\phi_{-}^{\prime}(u)\right| \leqq m$ for $u \geqq u_{0}$, there is an $x_{0}$, depending on $u_{0}$, such that for $x_{0} \leqq x_{1} \leqq x_{2}$

$$
x_{2}-x_{1} \leqq \pi \int_{u_{1}}^{u_{2}} \frac{1+\psi^{\prime 2}(u)}{\theta(u)} d u+\frac{\pi}{12} \int_{u_{1}}^{u_{2}} \frac{\theta^{\prime 2}(u)}{\theta(u)} d u+8 \pi\left(1+\frac{4}{3} m^{2}\right) .
$$

(b) If $S$ is an L-strip with the boundary inclination $\gamma=0$ at $u=+\infty$, then

$$
x_{2}-x_{1} \leqq \pi \int_{u_{1}}^{u_{2}} \frac{1+\psi^{\prime 2}(u)}{\theta(u)} d u+\frac{\pi}{12} \int_{u_{1}}^{u_{2}} \frac{\theta^{\prime 2}(u)}{\theta(u)} d u+o(1)
$$

as $x_{1} \rightarrow+\infty$, uniformly with respect to $y_{1}, y_{2}$.

REMARK. Ahlfors proves [1, pp. 12-16] the following theorem. Let $S$ be a region represented in the form $-\theta(u) / 2<v<\theta(u) / 2,-\infty<u<+\infty$, where $\theta(u)$ is of bounded variation in any finite interval and $0<\theta(u)<L$ for all $u$. Then, if $x_{1}, x_{2}, u_{1}, u_{2}$ have the same meaning as in Theorem IV,

$$
x_{2}-x_{1} \leqq \pi \int_{u_{1}}^{u_{2}} \frac{d u}{\theta(u)}+8 \pi+16 \pi L^{2} \frac{\theta_{m}^{2}+V}{\theta_{m}^{4}},
$$

where $\theta_{m}$ is the infimum of $\theta(u)$ for $u_{1}-4 L \leqq u \leqq u_{2}+4 L$ and $V$ is the total variation of $\theta^{2}(u)$ in this interval. (It should be noted that $\psi(u) \equiv 0$ here because $S$ is symmetrical with respect to the real axis.) While the hypotheses of Theorem IV regarding the smoothness of the boundary of $S$ are more stringent than those of Ahlfors' theorem, Theorem IV contains no restriction as to the symmetry of $S$ or boundedness of $\theta(u)$. Moreover, since $V=2 \int_{u=u_{1}-4 L}^{u_{2}+4 L} \theta(u)$ $|d \theta(u)|$, for continuous $\theta(u)$,

$$
16 \pi L^{2} \frac{\theta_{m}^{2}+V}{\theta_{m}^{4}} \geqq 16 \pi \frac{L^{2}}{\theta_{m}^{2}}\left\{1+2 \int_{u=u_{1}-4 L}^{u=u_{2}+4 L} \frac{|d \theta(u)|}{\theta(u)}\right\},
$$


and this shows that under the hypotheses of Theorem IV (b) the "remainder" term $\pi \int_{u_{1}}^{u_{2}}\left(\theta^{\prime 2}(u) / \theta(u)\right) d u+o(1)$ is smaller than the one in (5.3) if $u_{1}$ is sufficiently large. This becomes important in the case when $\lim \inf _{u \rightarrow+\infty} \theta(u)=0$, since then (5.4) is not bounded as $u_{2} \rightarrow+\infty\left(u_{1}\right.$ fixed), while $\int_{u_{1}}^{\infty}\left(\theta^{\prime 2}(u) / \theta(u)\right) d u$ converges for very general classes of functions $\theta(u)$. - In the proof of Theorem IV (as well as in that of Theorem VI below) we make use of Ahlfors' method of relating area and arc length employed in the proof of his inequalities.

6. Proof of Theorem IV. 1. Let for $x_{1} \leqq x \leqq x_{2}, a<U(z)<b$. We assume first that the functions $\phi_{+}(u)$ and $\phi_{-}(u)$ have two continuous derivatives for $a \leqq u \leqq b$.

The functions

$$
\bar{u}=h(u)=\int_{u_{0}}^{u} \frac{\eta d t}{\left(\theta(t)\left(1+t^{2}\right)\right)^{1 / 2}}, \quad \bar{v}=\frac{v-\psi(u)}{\theta(u)}, \quad \eta>0, \text { a constant },
$$

map the domain $S(a, b):\left\{\phi_{-}(u) \leqq v \leqq \phi_{+}(u), a \leqq u \leqq b\right\}$ in a one-to-one and continuous manner onto a rectangle: $\{\bar{a} \leqq \bar{u} \leqq \bar{b},|\bar{v}| \leqq 1\}$ of the $w \bar{w}$-plane, $\bar{w}=\bar{u}+i \bar{v}$. The function $W(z)$ maps the line segment $\{x,|y| \leqq \pi / 2\}, x_{1} \leqq x \leqq x_{2}$, onto a rectifiable $\operatorname{arc} l_{x}$ in $S$ and the transformation (6.1) carries $l_{x}$ into a rectifiable arc $\bar{l}_{x}$ within the strip $|\bar{v}| \leqq 1$ of the $w$-plane. If the variable arc lengths of $l_{x}$ and $\bar{l}_{x}$ are denoted by $s$ and $\bar{s}$, respectively, the

$$
\text { length of } \bar{l}_{x}=\int_{l_{x}} \frac{d \bar{s}}{d s} d s=\int_{-\pi / 2}^{\pi / 2} \frac{d \bar{s}}{d s}\left|W^{\prime}(x+i y)\right| d y .
$$

Since $\bar{l}_{x}$ connects a point on the line $\bar{v}=+1$ with another on $\bar{v}=-1$, its length is greater than or equal to 1 . Hence,

$$
1 \leqq\left\{\int_{-\pi / 2}^{\pi / 2} \frac{d \bar{s}}{d s}\left|W^{\prime}(x+i y)\right| d y\right\}^{2} \leqq \pi \int_{-\pi / 2}^{\pi / 2}\left(\frac{d \bar{s}}{d s}\right)^{2}\left|W^{\prime}(x+i y)\right|^{2} d y .
$$

Now, if $u(s), v(s)$ denote the parametric representation of $l_{x}$ in terms of $s$,

$$
\begin{aligned}
\left(\frac{d \bar{s}}{d s}\right)^{2}= & \left(\frac{d \bar{u}}{d u} \frac{d u}{d s}\right)^{2}+\left(\frac{\partial \bar{v}}{\partial u} \frac{d u}{d s}+\frac{\partial \bar{v}}{\partial v} \frac{d v}{d s}\right)^{2} \\
= & {\left[h^{\prime 2}(u)+\left(\frac{\partial \bar{v}}{\partial u}\right)^{2}\right]\left(\frac{d u}{d s}\right)^{2}+2 \frac{\partial \bar{v}}{\partial u} \frac{\partial \bar{v}}{\partial v} \frac{d u}{d s} \frac{d v}{d s}+\left(\frac{\partial \bar{v}}{\partial v}\right)^{2}\left(\frac{d v}{d s}\right)^{2} } \\
\leqq & {\left[h^{\prime 2}(u)+\left(\frac{\partial \bar{v}}{\partial u}\right)^{2}\right]\left(\frac{d u}{d s}\right)^{2} } \\
& +2\left|\frac{\partial \bar{v}}{\partial v}\right|\left|\frac{d u}{d s}\right|\left|\frac{d v}{d s}\right|\left(h^{\prime 2}(u)+\left(\frac{\partial \bar{v}}{\partial u}\right)^{2}\right)^{1 / 2}+\left(\frac{\partial \bar{v}}{\partial v}\right)^{2}\left(\frac{d v}{d s}\right)^{2} \\
= & \left\{\left(h^{\prime 2}(u)+\left(\frac{\partial \bar{v}}{\partial u}\right)^{2}\right)^{1 / 2} \frac{d u}{d s}+\left|\frac{\partial \bar{v}}{\partial v}\right|\left|\frac{d v}{d s}\right|\right\}^{2} .
\end{aligned}
$$


Hence, by Schwarz's inequality,

$$
\begin{aligned}
\left(\frac{d \bar{s}}{d s}\right)^{2} & \leqq\left[h^{\prime 2}(u)+\left(\frac{\partial \bar{v}}{\partial u}\right)^{2}+\left(\frac{\partial \bar{v}}{\partial v}\right)^{2}\right]\left[\left(\frac{d u}{d s}\right)^{2}+\left(\frac{d v}{d s}\right)^{2}\right] \\
& =h^{\prime 2}(u)+\left(\frac{\partial \bar{v}}{\partial u}\right)^{2}+\left(\frac{\partial \bar{v}}{\partial v}\right)^{2},
\end{aligned}
$$

since $(d u / d s)^{2}+(d v / d s)^{2}=1$. Observing that (we leave off the argument $u$ )

$$
\frac{\partial \bar{v}}{\partial u}=-\frac{\theta \psi^{\prime}+(v-\psi) \theta^{\prime}}{\theta^{2}}, \quad \frac{\partial \bar{v}}{\partial v}=\frac{1}{\theta}
$$

$\left(\psi^{\prime}, \theta^{\prime}\right.$ are derivatives with respect to $\left.u\right)$, we find at every point $(u, v)$ of $l_{x}$

$$
\left(\frac{d \bar{s}}{d s}\right)^{2} \leqq \frac{\eta^{2}}{\theta \cdot\left(u^{2}+1\right)}+\frac{\theta^{2} \psi^{\prime 2}+2(v-\psi) \theta \theta^{\prime} \psi^{\prime}+(v-\psi)^{2} \theta^{\prime 2}}{\theta^{4}}+\frac{1}{\theta^{2}} \equiv f(u, v) .
$$

Hence, from (6.2)

$$
1 \leqq \pi \int_{-\pi / 2}^{\pi / 2} f(U(z), V(z))\left|W^{\prime}(x+i y)\right|^{2} d y .
$$

Integrating this inequality between the limits $x_{1}, x_{2}$ with respect to $x$, we find

$$
\left(x_{2}-x_{1}\right) \leqq \pi \int_{x_{1}}^{x_{2}} d x \int_{-\pi / 2}^{\pi / 2} f(U(z), V(z))\left|W^{\prime}(x+i y)\right|^{2} d y .
$$

Now, introducing the variables $(u, v)$ in place of $(x, y)$ by means of the transformation $w=W(x+i y)$, we obtain

$$
x_{2}-x_{1} \leqq \pi \iint_{(T)} f(u, v) d u d v
$$

where $T$ is the image of the rectangle $x_{1}<x<x_{2},|y|<\pi / 2$, in the $w$-plane. If $u_{* 1}=\min _{|y| \leqq \pi / 2} U\left(x_{1}+i y\right)$ and $u_{2}^{*}=\max _{|y| \leqq \pi / 2} U\left(x_{2}+i y\right)$, it is clear that $T$ is contained in the region $\phi_{-}(u)<v<\phi_{+}(u), u_{* 1}<u<u_{2}^{*}$. Using this fact and substituting the value in $(6.3)$ for $f(u, v)$, we find

$$
\begin{aligned}
x_{2}-x_{1} \leqq & \pi \int_{u *_{1}}^{u_{2}^{*}} \int_{\phi_{-}(u)}^{\phi_{+}(u)} \frac{1+\psi^{\prime 2}(u)}{\theta^{2}(u)} d v d u+\pi \eta^{2} \int_{u * 1}^{u_{2}^{*}} \int_{\phi_{-}(u)}^{\phi_{+}(u)} \frac{d v d u}{\theta(u)\left(u^{2}+1\right)} \\
& +\pi \int_{u *_{1}}^{u_{2}^{*}} \int_{\phi_{-}(u)}^{\phi_{+}(u)} \frac{2(v-\psi) \theta \theta^{\prime} \psi^{\prime}+(v-\psi)^{2} \theta^{\prime 2}}{\theta^{4}} d v d u .
\end{aligned}
$$

The integration with respect to $v$ can be easily carried through. The first two integrals yield the result 


$$
\pi \int_{u_{* 1}}^{u_{2}^{*}} \frac{1+\psi^{\prime 2}(u)}{\theta(u)} d u+\pi \eta^{2} \int_{u_{* 1}}^{u_{2}^{*}} \frac{d u}{1+u^{2}} .
$$

The third integral is equal to

$$
2 \pi \int_{u_{* 1}}^{u_{2}^{*}} 0 \cdot \frac{\theta^{\prime} \psi^{\prime}}{\theta^{3}} d u+\pi \int_{u_{* 1}}^{u_{2}^{*}} \frac{2}{3}\left(\frac{\theta}{2}\right)^{3} \frac{\theta^{\prime 2}}{\theta^{4}} d u=\frac{\pi}{12} \int_{u_{* 1}}^{u_{2}^{*}} \frac{\theta^{\prime 2}(u)}{\theta(u)} d u .
$$

Hence we finally obtain

$$
x_{2}-x_{1} \leqq \pi \int_{u_{*} 1}^{u_{2}^{*}} \frac{1+\psi^{\prime 2}(u)}{\theta(u)} d u+\frac{\pi}{12} \int_{u_{* 1}}^{u_{2}^{*}} \frac{\theta^{\prime 2}(u)}{\theta(u)} d u+\pi \eta^{2} \int_{-\infty}^{\infty} \frac{d u}{1+u^{2}} .
$$

This inequality is true for every $\eta$. Keeping $x_{1}, x_{2}$ fixed and letting $\eta \rightarrow 0$ we find

$$
x_{2}-x_{1} \leqq \pi \int_{u_{* 1}}^{u_{2}^{*}} \frac{1+\psi^{\prime 2}(u)}{\theta(u)} d u+\frac{\pi}{12} \int_{u_{* 1}}^{u_{2}^{*}} \frac{\theta^{\prime 2}(u)}{\theta(u)} d u .
$$

2. If the hypothesis made at the beginning of part 1 of this proof (that $\phi_{+}(u)$ and $\phi_{-}(u)$ have continuous second derivatives for $\left.a \leqq u \leqq b\right)$ is not satisfied, we replace the $\operatorname{arcs} \beta_{+}: v=\phi_{+}(u)$ and $\beta_{-}: v=\phi_{-}(u), a \leqq u \leqq b^{\prime}\left(b^{\prime}>b\right)$ by certain $\operatorname{arcs} \beta_{+}^{(n)}$ and $\beta_{-}^{(n)}$ for which this assumption is true and which converge to $\beta_{+}$and $\beta_{-}$, respectively, as $n \rightarrow+\infty$. We proceed as follows:

Since $\phi_{+}(u)$ and $\phi_{-}(u)$ have bounded difference quotients, $\phi_{+}{ }^{\prime}(u)$ and $\phi_{-}{ }^{\prime}(u)$ exist almost everywhere in $a \leqq u \leqq b^{\prime}$ and are, in absolute value, less than or equal to $m$. There exist therefore two sequences of continuous functions, $\phi_{+}{ }^{\prime}(u ; n)$ and $\phi_{-}^{\prime}(u ; n), a \leqq u \leqq b$, such that

$$
\int_{a}^{b}\left|\phi_{+}^{\prime}(u ; n)-\phi_{+}^{\prime}(u)\right| d u \rightarrow 0, \int_{a}^{b}\left|\phi_{-}^{\prime}(u ; n)-\phi_{-}^{\prime}(u)\right| d u \rightarrow 0 \text { as } n \rightarrow \infty,
$$

$$
\begin{aligned}
& \left|\phi_{+}^{\prime}(u ; n)\right| \leqq 2 m,\left|\phi_{-}^{\prime}(u ; n)\right| \leqq 2 m, \quad a \leqq u \leqq b, n=1,2,3, \cdots, \\
& \frac{d}{d u} \phi_{+}^{\prime}(u ; n), \frac{d}{d u} \phi_{-}^{\prime}(u ; n) \quad \text { exist and are continuous for } a \leqq u \leqq b .
\end{aligned}
$$

Now we define

$$
\begin{array}{r}
\phi_{+}(u ; n)=\phi_{+}(a)+\int_{a}^{u} \phi_{+}^{\prime}(t ; n) d t, \phi_{-}(u ; n)=\phi_{-}(a)+\int_{a}^{u} \phi_{-}^{\prime}(t ; n) d t, \\
a \leqq u \leqq b .
\end{array}
$$

Furthermore, we may assume that $\phi_{+}(b) \neq 0$ and $\phi_{-}(b) \neq 0$ and set for $b \leqq u \leqq b^{\prime}$

$$
q_{+}(u ; n)=q_{n}+\frac{1-q_{n}}{b^{\prime}-b}(u-b) \text { where } q_{n}=\frac{\phi_{+}(b ; n)}{\phi_{+}(b)},
$$


and define

$$
q_{-}(u ; n)=p_{n}+\frac{1-p_{n}}{b^{\prime}-b}(u-b) \text { where } p_{n}=\frac{\phi_{-}(b ; n)}{\phi_{-}(b)},
$$

$$
\phi_{+}(u ; n)=\phi_{+}(u) q_{+}(u ; n), \quad \phi_{-}(u ; n)=\phi_{-}(u) q_{-}(u ; n) \quad \text { for } b \leqq u \leqq b^{\prime} .
$$

Then it is evident from (6.5.1), (6.6) and (6.7) that the arcs

$$
\beta_{+}^{(n)}: v=\phi_{+}(u ; n) \text { and } \beta_{-}^{(n)}: v=\phi_{-}(u ; n), \quad a \leqq u \leqq b^{\prime},
$$

converge to the arcs $\beta_{+}$and $\beta_{-}$, respectively, in the sense that

(6.8) $\lim _{n \rightarrow \infty} \phi_{+}(u ; n)=\phi_{+}(u), \quad \lim _{n \rightarrow \infty} \phi_{+}(u ; n)=\phi_{-}(u)$, uniformly for $a \leqq u \leqq b^{\prime}$.

If now, for sufficiently large $n$, the arcs $\beta_{+}$and $\beta_{-}$are replaced by $\beta_{+}^{(n)}$ and $\beta_{-}^{(n)}$, respectively, we obtain a Jordan strip $S_{n}$ whose boundary curves satisfy the hypotheses stated in the theorem and in the beginning of part 1 of this proof. Let $W_{n}(z)$ be the function which maps the strip $|y|<\pi / 2$ onto $S_{n}$ in such a manner that $x=+\infty$ corresponds to $u=+\infty$ and that $W_{n}(0)=W(0)$. Then we may define $u_{* 1}^{(n)}$ and $u_{2}^{*(n)}$ for $W_{n}(z)$ in the same way as $u_{* 1}$ and $u_{2}^{*}$ are defined for $W(z)$. Since $\beta_{+}^{(n)}$ and $\beta_{-}^{(n)}$ converge to $\beta_{+}$and $\beta_{-}$, respectively, in the above specified manner,

$$
\lim _{n \rightarrow \infty} u_{* 1}^{(n)}=u_{* 1}, \quad \lim _{n \rightarrow \infty} u_{2}^{*(n)}=u_{2}^{*}\left({ }^{12}\right) .
$$

Let $\theta_{n}(u)$ and $\psi_{n}(u)$ have the same meaning for $S_{n}$ as $\theta(u)$ and $\psi(u)$ for $S$. We now apply (6.4) to the region $S_{n}^{*}$, where $n$ is sufficiently large, and obtain an analogous inequality in which $\theta, \psi^{\prime}, \theta^{\prime}, u_{* 1}$, and $u_{2}^{*}$ are replaced by $\theta_{n}, \psi_{n}^{\prime}, \theta_{n}^{\prime}, u_{* 1}^{(n)}, u_{2}^{*(n)}$, respectively. Here we let $n \rightarrow \infty$, and it follows from (6.5.1) and (6.5.2), that the inequality holds for the original region $S$ without the assumption of the existence of the second derivatives of $\phi_{+}(u)$ and $\phi_{-}(u)$.

(12) To see this, we may assume that $w=0$ is neither in $S$ nor on the boundary of $S$ and transform $S$ and $S_{n}$ by means of the function $\omega=1 / w$ into limited regions $R$ and $R_{n}$, respectively. $R$ and $R_{n}$ are bounded by closed curves $\Gamma$ and $\Gamma_{n}$, respectively, which coincide except for the arcs $\gamma_{+}^{(n)}$ and $\gamma_{-}^{(n)}$ of $\Gamma_{n}$, the images of $\beta_{+}^{(n)}$ and $\beta_{-}^{(n)}$, and $\gamma_{+}$and $\gamma_{-}$of $\Gamma$, the images of $\beta_{+}$and $\beta_{-}$. From (6.8) it follows that the curves $\Gamma_{n}$ converge to $\Gamma$ in the sense that their Fréchet distance approaches 0 . (The Fréchet distance $d$ of two Jordan curves $C$ and $C^{\prime}$ is defined as follows: For any continuous one-to-one transformation of $C$ onto $C^{\prime}$, the distance of corresponding points has a maximum. The greatest lower bound of these maxima for all possible transformations is $d$.) If $\omega_{n}(\zeta)$ and $\omega(\zeta)$ map $|\zeta|<1$ conformally onto $R_{n}$ and $R$, respectively, and if $\omega_{n}(0)=\omega(0)$ and $\omega_{n}(1)=\omega(1)=0$ (the image of $u=+\infty$ ), then it follows from a theorem of Rado [1, pp. 180 $186]$ that $\omega_{n}(\zeta) \rightarrow \omega(\zeta)$ uniformly in $|\zeta| \leqq 1$ as $n \rightarrow \infty$. Now the functions $W_{n}(z)$ and $W(z)$ of the text are

$$
W_{n}(z)=\left[\omega_{n}(\zeta)\right]^{-1}, \quad W(z)=[\omega(\zeta)]^{-1}, \quad \text { where } z=\log \frac{1+\zeta}{1-\zeta},
$$

and it follows therefore that $W_{n}(z) \rightarrow W(z)$ uniformly in any fixed rectangle $\xi_{1} \leqq x \leqq \xi_{2},|y| \leqq \pi / 2$. This implies (6.9). 
3. Now the proof is easily completed. We treat the cases (a) and (b) of our theorem separately.

(a) By Theorem III (a),

$$
\pi \int_{u_{* 1}}^{u_{1}} \frac{d u}{\theta(u)} \leqq x_{1}-x_{1}+4 \pi=4 \pi, \quad \pi \int_{u_{2}}^{u_{2}^{*}} \frac{d u}{\theta(u)} \leqq 4 \pi .
$$

Moreover, since

$$
1+\psi^{\prime 2}(u) \leqq 1+m^{2}, \quad \theta^{\prime 2}(u) \leqq 4 m^{2},
$$

we find (5.1) from (6.4).

(b) In the case (b), we have by Theorem III (b),

$$
\int_{u_{1}}^{u_{1}} \frac{d u}{\theta(u)}=o(1), \quad \int_{u_{2}}^{u_{2}^{*}} \frac{d u}{\theta(u)}=o(1)
$$

as $u_{1} \rightarrow+\infty$, and by hypothesis

$$
\lim _{u \rightarrow+\infty} \psi^{\prime}(u)=\lim _{n \rightarrow+\infty} \theta^{\prime}(u)=0 .
$$

From these facts (5.2) follows immediately.

7. A corollary of Theorem IV. If $S$ is an L-strip with the boundary inclination $\gamma=0$ at $u=+\infty$, for which the integrals

$$
\int_{u_{0}}^{\infty} \frac{\phi_{+}^{\prime 2}(u)}{\theta(u)} d u \text { and } \int_{u_{0}}^{\infty} \frac{\phi_{-}^{\prime 2}(u)}{\theta(u)} d u \text { converge, }
$$

and if $z=Z(w)=X(w)+i Y(w)$ maps $S$ conformally onto the strip $|y|<\pi / 2$, in such a manner that $\lim _{u \rightarrow+\infty} X(w)=+\infty$, then there exists a constant $\lambda$ such that, for $w=u+i v \in S$,

(7.2) $X(w)=\lambda+\pi \int_{u_{0}}^{u} \frac{d t}{\theta(t)}+o(1)$ as $u \rightarrow+\infty$, uniformly with respect to $v$.

Proof. First we note that (7.1)implies the convergence of $\int_{u_{0}}^{\infty}\left(\theta^{\prime 2}(u) / \theta(u)\right) d u$ and $\int_{u_{0}}^{\infty}\left(\psi^{\prime 2}(u) / \theta(u)\right) d u$. Applying now Theorems III (b) and IV (b) we find that, for $w_{1}=u_{1}+i v_{1}, w_{2}=u_{2}+i v_{2}$ in $S, u_{1} \leqq u_{2}$,

$$
\pi \int_{u_{1}}^{u_{2}} \frac{d t}{\theta(t)}+o(1) \leqq X\left(w_{2}\right)-X\left(w_{1}\right) \leqq \pi \int_{u_{1}}^{u_{2}} \frac{d t}{\theta(t)}+o(1),
$$

where $o(1) \rightarrow 0$ as $u_{1}, u_{2} \rightarrow+\infty$, uniformly with respect to $v_{1}, v_{2}$. If we set $A(w) \equiv X(w)-\pi \int_{u_{0}}^{u}[\theta(t)]^{-1} d t$, then (7.3) means that for every $\epsilon$ there exists an $N(\epsilon)$ such that 


$$
\left|A\left(w_{2}\right)-A\left(w_{1}\right)\right|<\epsilon \quad \text { if } u_{2} \geqq u_{1}>N(\epsilon) .
$$

Hence (7.2) is true by Cauchy's convergence principle.

The condition (7.1) is very restrictive, since $\theta(u)$ might be of smaller order of magnitude than $\phi_{+}{ }^{\prime 2}(u)$ and $\phi_{-}{ }^{\prime 2}(u)$. Our next aim is, therefore, to establish, in place of Theorem III (b), a lower bound for the difference $x_{2}-x_{1}$ in terms of the integral $\int_{u_{1}}^{u_{2}}\left\{\left(1+\psi^{\prime 2}(u)\right) / \theta(u)\right\} d u$ and of a suitable "remainder" term. This inequality, combined with Theorem IV, will yield an asymptotic representation for $X(w)$ which will require only the existence of the integral $\int_{u_{0}}^{\infty}\left(\theta^{\prime 2}(u) / \theta(u)\right) d u$ in place of $(7.1)$.

\section{The SECOND BASIC INEQUALITY}

8. Preliminaries. In order to derive the inequality indicated at the end of $\$ 7$ we shall establish several preliminary results.

(a) Let $S$ be an $L$-strip in the $w$-plane with the boundary inclination $\gamma$, $-\pi / 2 \leqq \gamma \leqq \pi / 2$, at $u=+\infty$. Moreover, let its boundary curves $C_{+}: v=\phi_{+}(u)$ and $C_{-}: v=\phi_{-}(u), u \geqq u_{0}$, satisfy the hypothesis that $\phi_{+}^{\prime}(u)$ and $\phi_{-}^{\prime}(u)$ are continuous and of bounded variation in any finite interval contained in $u \geqq u_{0}$.

(b) Let $s$ denote the variable arc length of $C_{-}$measured from $u=u_{0}$ and $w=w(s)$ the parametric representation of $C_{-}$by means of $s$ as parameter. Let $\alpha(s),-\pi / 2<\alpha(s)<\pi / 2$, be the angle of inclination of the tangent to $C_{-}$at the point $w(s)$.

(c) Since the boundary inclination of $S$ at $u=+\infty$ is $\gamma$, there exists a half-plane $H_{c}: u \geqq c$, such that the angle of inclination of a tangent at any point of $C_{+}$or $C_{-}$in $H_{c}$ satisfies the relation

$$
|\alpha-\gamma|<\frac{\pi}{8} \text {. }
$$

Let $C_{+}{ }^{*}$ and $C_{-}^{*}$ denote the parts of $C_{+}$and $C_{-}$, respectively, which lie in $H_{c}$. Application of the mean value theorem shows then that any straight line with the angle of inclination $\beta$, where

$$
\left|\beta-\left(\gamma+\frac{\pi}{2}\right)\right|<\frac{\pi}{8},
$$

can intersect $C_{+}{ }^{*}$ and $C_{-}{ }^{*}$ in but one point each. Hence, there exists a number $\sigma>0$ such that any normal to $C_{-}$at $w(s)$, where $s \geqq \sigma$, will intersect $C_{+}{ }^{*}$ and $C_{-}{ }_{-}$in exactly one point each. The segment of this normal which lies in $S$ will be denoted by $\Delta_{s}$, its length by $\Delta(s)$. Evidently $\Delta(s)$ is a continuous function for $s \geqq \sigma$.

Denote the point $w(s)$ by $A$, the other end point of $\Delta_{s}$ (on $C_{+}$) by $B$ and the point $u+i \phi_{+}(u)$ by $C(u=R w(s))$. Since, in the triangle $A B C$, the angle $A$ approaches $|\gamma|$ and $B$ approaches $\pi / 2$ as $s \rightarrow \infty$, it follows by the law of sines that 


$$
\lim _{u \rightarrow+\infty} \frac{\Delta(s)}{\theta(u)}=\cos \gamma, \quad u=R w(s) .
$$

(d) For the following two lemmas we assume that hypothesis (a) is satisfied.

Lemma 2. If $\alpha^{\prime}(s)$ is continuous for $\sigma \leqq a \leqq s \leqq b$, and if $\Delta(s) \alpha^{\prime}(s)<1$ in the open interval $a<s<b$, then no two normals $\Delta_{s}, a \leqq s \leqq b$, intersect in $\bar{S}\left({ }^{13}\right)$.

Proof. We show first that no two normals $\Delta_{s}$ of any closed subarc $a<\alpha \leqq s \leqq \beta<b$ of the arc $a<s<b$, intersect. If this assertion were not true, there would exist a point $A_{1}\left(s=a_{1}\right)$ and a point $B_{1}\left(s=b_{1}\right)$ on $C_{-}, \alpha \leqq a_{1}<b_{1} \leqq \beta$, such that $\Delta_{a_{1}}$ and $\Delta_{b_{1}}$ would intersect at some point $D_{1} \in \bar{S}\left({ }^{13}\right)$. Let $I_{1}$ be the arc $a_{1} \leqq s \leqq b_{1}$. The curvilinear triangle $A_{1} B_{1} D_{1}$ lies entirely within $\bar{S}$. Let $s^{\prime}=(1 / 2)\left(a_{1}+b_{1}\right)$. Then $\Delta_{z^{\prime}}$ enters this triangle at the point $s=s^{\prime}$ of $C_{-}$and will intersect either $A_{1} D_{1}$ or $B_{1} D_{1}$ or both at some point $D_{2} \in \bar{S}$. If $D_{2}$ lies on $A_{1} D_{1}$, we let $I_{2}$ be the arc $a_{2} \leqq s \leqq s^{\prime}$ and denote its end points by $A_{2}\left(s=a_{2}\right)$ and $B_{2}\left(s=b_{2}\right)$ where $a_{2}=a_{1}, b_{2}=s^{\prime}$. Then $\Delta_{8^{\prime \prime}}$ where $s^{\prime \prime}=(1 / 2)\left(a_{2}+b_{2}\right)$, will intersect either $A_{2} D_{2}$ or $B_{2} D_{2}$ or both at some point $D_{3}$. If $D_{2}$ does not lie on $A_{1} D_{1}$, let $I_{2}$ be the arc $s^{\prime} \leqq s \leqq b$ where now $a_{2}=s^{\prime}$ and $b_{2}=b_{1}$ and the end points of $I_{2}$ are again denoted by $A_{2}$ and $B_{2}$. Everything said about $\Delta_{g^{\prime \prime}}$ holds then for this second choice of $I_{2}$. Continuing in the manner indicated, we obtain a sequence of intervals $I_{n}(n=1,2,3, \cdots)$ whose end points we shall denote by $A_{n}\left(s=a_{n}\right)$ and $B_{n}\left(s=b_{n}\right), \alpha \leqq a_{n}<b_{n} \leqq \beta$, such that $\lim _{n \rightarrow \infty} a_{n}=\lim _{n \rightarrow \infty} b_{n}=c$ exist and that $\Delta_{a_{n}}$ and $\Delta_{b_{n}}$ intersect at some point $D_{n} \in \bar{S}$. By the law of sines, we obtain from the triangle $A_{n} B_{n} D_{n}$ :

$$
\frac{\sin D_{n}}{A_{n} B_{n}}=\frac{\sin A_{n}}{B_{n} D_{n}}
$$

Since the angle $A_{n}$ approaches $\pi / 2$ as $n \rightarrow \infty$ and the angle $D_{n}=\alpha\left(b_{n}\right)-\alpha\left(a_{n}\right)$ $\geqq 0$, and since $B_{n} D_{n} \leqq \Delta\left(b_{n}\right)$ we find

or

$$
\lim _{n \rightarrow \infty} \frac{\alpha\left(b_{n}\right)-\alpha\left(a_{n}\right)}{b_{n}-a_{n}} \geqq \lim _{n \rightarrow \infty} \frac{\sin A_{n}}{\Delta\left(b_{n}\right)}=\frac{1}{\Delta(c)}
$$

$$
\alpha^{\prime}(c) \Delta(c) \geqq 1,
$$

which contradicts the hypothesis.

To complete the proof of the lemma we must show that no $\Delta_{s}, a<s<b$, intersects $\Delta_{a}$ or $\Delta_{b}$. Suppose $\Delta_{s_{0}}, a<s_{0}<b$, intersected $\Delta_{b}$ at some point $D_{0}$. Then any $\Delta_{s_{1}}$ with $s_{0}<s_{1}<b$ must intersect $\Delta_{b}$, since it must intersect $\Delta_{s_{0}}$ or $\Delta_{b}$ and it cannot intersect $\Delta_{s_{0}}$ (by the part already proved). Let $B$ denote the point $s=b$ on $C_{-}$. The point $D_{1}$ at which $\Delta_{s_{1}}$ intersects $\Delta_{b}$ must lie on the seg-

(13) The closure of a point set $M$ in the complex plane will be denoted by $\bar{M}$. 
ment $B D_{0}$ of $\Delta_{b}$ (for otherwise $\Delta_{s_{1}}$ would meet $\Delta_{s_{0}}$ ) and hence $B D_{1}<B D_{0}$ $\leqq \Delta(b)$.

Let now $\left\{s_{n}\right\}, n=1,2,3, \cdots, s_{0}<s_{n}<s_{n+1}<b$, denote a sequence which converges to $b$ as $n \rightarrow \infty$, and let $\Delta_{s_{n}}$ intersect $\Delta_{b}$ at $D_{n}$. For all $n, B D_{n} \leqq B D_{1}$ $<\Delta(b)$. Call $A_{n}$ the point $s=s_{n}$ on $C_{-}$. Then, by the law of sines,

$$
\frac{\sin D_{n}}{A_{n} B}=\frac{\sin A_{n}}{B D_{n}} \geqq \frac{\sin A_{n}}{B D_{1}} \text {. }
$$

Since the angle $A_{n} \rightarrow \pi / 2$ as $n \rightarrow \infty$ and since $D_{n}=\alpha(b)-\alpha\left(s_{n}\right)$,

$$
\lim _{n \rightarrow \infty} \frac{\alpha(b)-\alpha\left(s_{n}\right)}{b-s_{n}} \geqq \frac{1}{B D_{1}}>\frac{1}{\Delta(b)}, \quad \text { or } \quad \Delta(b) \alpha^{\prime}(b)>1,
$$

while the continuity of $\Delta(s) \cdot \alpha^{\prime}(s)$ implies that $\Delta(b) \alpha^{\prime}(b) \leqq 1$. A similar argument applies to the point $s=a$.

Lemma 3. Let $\Delta_{a}$ and $\Delta_{b}, \sigma \leqq a<b$, intersect at a point $C$ in $\bar{S}$. Suppose that any of the angles, which a chord of $C_{-}$or of $C_{+}$through the end points $\left.{ }^{14}\right)$ of $\Delta_{s}$ and $\Delta_{s^{\prime}}, a \leqq s<s^{\prime} \leqq b$, forms with $\Delta_{s}$ and $\Delta_{s^{\prime}}$, differs from $\pi / 2$ in absolute value by less than $\epsilon, 0<\epsilon<\pi / 8\left({ }^{15}\right)$. Then

$$
\int_{a}^{b} \frac{d s}{\Delta(s)} \leqq \frac{\alpha(b)-\alpha(a)}{\cos ^{3}(2 \epsilon)} .
$$

Proof. Call the points $s=a$ and $s=b$ on $C_{-}, A$ and $B$, respectively. Draw $\Delta_{s}$, $a<s<b$, and denote its end points on $C_{-}$and $C_{+}$by $D$ and $E$, respectively. $D E$ will intersect either $A C$ or $B C$ or both. Suppose it intersects $B C$ at a point $F$. Call $C^{\prime}$ the end point of $\Delta_{b}$ on $C_{+}$. From the two triangles $B D F$ and $C^{\prime} E F$ we find by the law of sines

$$
D F=B F \frac{\sin B}{\sin D}, \quad F E=F C^{\prime} \frac{\sin C^{\prime}}{\sin E} .
$$

Since, by hypothesis, $\sin B>\sin (\pi / 2-\epsilon)=\cos \epsilon, \sin C^{\prime}>\cos \epsilon$, we have

$$
\Delta(s)=D F+F E>B C^{\prime} \cos \epsilon=\Delta(b) \cos \epsilon .
$$

If $\Delta_{s}$ intersects $\Delta_{a}$ we obtain in a similar way

$$
\Delta(s) \geqq \Delta(a) \cos \epsilon .
$$

Now, by the mean value theorem, $\int_{a}^{b}[\Delta(s)]^{-1} d s=(b-a) / \Delta(\bar{s})$ where $a<\bar{s}<b$. Hence, by (8.3) or (8.4)

(14) If the end points of $\Delta_{s}$ and $\Delta_{s^{\prime}}$ on $C_{+}$coincide at a point $P$, then a chord through these end points on $C_{+}$means the tangent to $C_{+}$at $P$.

(15) This condition will always be satisfied if $a$ is sufficiently large (because of the hypothesis in $\S 8(a))$. 


$$
\int_{a}^{b} \frac{d s}{\Delta(s)} \leqq(b-a) \frac{1}{\Delta(a) \cos \epsilon}
$$

or

$$
\int_{a}^{b} \frac{d s}{\Delta(s)} \leqq(b-a) \frac{1}{\Delta(b) \cos \epsilon}
$$

respectively.

From the triangle $A B C$ we find

$$
\Delta(a) \geqq A C=A B \frac{\sin B}{\sin C} \geqq A B \frac{\cos \epsilon}{\sin C}, \quad \Delta(b) \geqq A B \frac{\cos \epsilon}{\sin C},
$$

so that

$$
\int_{a}^{b} \frac{d s}{\Delta(s)} \leqq \frac{\sin C}{\cos ^{2} \epsilon} \frac{b-a}{A B} .
$$

Observing that the hypothesis regarding the chords of $C_{-}$implies that $\left({ }^{16}\right)$

$$
b-a \leqq \frac{A B}{\cos (2 \epsilon)}
$$

and that the angle $C=\alpha(b)-\alpha(a)$, we obtain (8.2).

9. "Invariant" formulation of the second basic inequality. We prove now

THEOREM V. Let $S$ be an L-strip which satisfies the hypothesis of $\$ 8$ (a) and let $\alpha(s), \Delta_{s}$, and $\Delta(s)$ be defined as in $\$ 8$ (b) and (c). Suppose that $z=Z(w)$ $=X(w)+i Y(w)$ maps $S$ conformally onto the strip $|y|<\pi / 2$ in such a manner that $X(w) \rightarrow+\infty$ as $u \rightarrow+\infty$. Let $w_{1}$ and $w_{2}$ be points in $\bar{S}$ and $\Delta_{s_{1}}$ and $\Delta_{s_{2}}$ normals to $C_{-}$which pass through $w_{1}$ and $w_{2}$, respectively $\left({ }^{17}\right)$. Let $x_{1}=X\left(w_{1}\right)$, $x_{2}=X\left(w_{2}\right)$. Then, if $s_{2}>s_{1}$,

$$
x_{2}-x_{1} \geqq \pi \int_{s_{1}}^{s_{2}} \frac{d s}{\Delta(s)}-\pi(1+o(1)) \int_{s_{-s_{1}}}^{s_{2}}|d \alpha(s)|+o(1)
$$

where the second integral on the right-hand side is taken in the sense of Stieltjes and $o(1) \rightarrow 0$ as $s_{1} \rightarrow+\infty$, uniformly in $w_{1}$ and $w_{2}$.

(16) The tangents to any two points of the arc $A B: a \leqq s \leqq b$, of $C$ form an angle not exceeding $2 \epsilon$. By the mean value theorem it follows therefore that the chord $A B$ forms an angle of measure less than $2 \epsilon$ with the tangent to any point of the arc $A B$. Hence, if the arc $A B$ is represented in the form $y=f(x), A B$ being the $x$-axis, $A$ the origin and $B$ the point $(l, 0)$, we have

$$
b-a=\int_{0}^{l}\left(1+\left[f^{\prime}(x)\right]^{2}\right)^{1 / 2} d x \leqq l\left(1+\tan ^{2}(2 \epsilon)\right)^{1 / 2}=\frac{A B}{\cos (2 \epsilon)} .
$$

${ }^{\left({ }^{17}\right)}$ If $R w_{1}$ and $R w_{2}$ are sufficiently large, there always exist normals $\Delta_{s_{1}}$ and $\Delta_{s_{1}}$ passing through $w_{1}$ and $w_{2}$, respectively. 
Proof. 1. It is sufficient to prove this theorem under the assumption that $\gamma=0$ since the statement of the theorem is invariant with respect to a rotation of the coordinate system in the $w$-plane through the angle $\gamma$ in the positive direction. Since $S$ is an $L$-strip with the boundary inclination $\gamma=0$ at $u=+\infty$, there exists for every $\epsilon>0, \epsilon<\pi / 8$, a half-plane $H_{c}: u \geqq c$, such that the angle of inclination $\alpha,|\alpha|<\pi / 2$, of the tangent at any point of $C_{+}$or $C_{-}$in $H_{c}$ satisfies the condition

$$
|\alpha|<\epsilon / 2 \text {. }
$$

Let an $\epsilon$ be fixed. We assume $s_{1}$ so large that all $\Delta_{s}$ with $s \geqq s_{1}$ lie entirely in $H_{c}$, say $s_{1}>\sigma_{1}$.

2. We assume first that $\alpha^{\prime}(s)$ exists and is continuous for $s_{1} \leqq s \leqq s_{2}$. Let

$$
x_{2}^{*}=\max _{w \in \Delta_{s_{2}}} X(w), \quad x_{* 1}=\min _{w \in \Delta_{\iota_{1}}} X(w) .
$$

Then we prove that

$$
x_{2}^{*}-x_{* 1} \geqq \pi \int_{s_{1}}^{s_{2}} \frac{d s}{\Delta(s)}-\frac{\pi}{\cos ^{3}(2 \epsilon)} \int_{s_{1}}^{s_{2}}\left|\alpha^{\prime}(s)\right| d s .
$$

Let $I_{1}: a_{1}<s<b_{1}$, be a largest open arc of $I: s_{1}<s<s_{2}$, where

$$
\Delta(s) \alpha^{\prime}(s)<1 .
$$

If there is no such arc we have on $I$

$$
\Delta(s) \alpha^{\prime}(s) \geqq 1
$$

and hence

$$
\pi \int_{s_{1}}^{s_{2}} \frac{d s}{\Delta(s)}-\pi \int_{s_{1}}^{s_{2}} \alpha^{\prime}(s) d s \leqq 0 \leqq x_{2}^{*}-x_{* 1}
$$

so that (9.2) is evidently true. If there are several such arcs, let $I_{1}$ be one of them. By Lemma 2, no two normals $\Delta_{8}$ of the closed arc $\bar{I}_{1}: a_{1} \leqq s \leqq b_{1}$, will intersect. If there are any normals $\Delta_{s}$ with $b_{1}<s \leqq s_{2}$ which intersect $\Delta_{b_{1}}$, let $\Delta_{b_{1}^{\prime}}^{\prime}$ be the one for which $b_{1}^{\prime}$ is as large as possible. Otherwise we set $b_{1}^{\prime}=b_{1}$. If there are any $\Delta_{s}$ with $s_{1} \leqq s<a_{1}$ which intersect $\Delta_{a_{1}}$, let $\Delta_{a_{1}^{\prime}}$ be the one for which $a_{1}^{\prime}$ is as small as possible. Otherwise we set $a_{1}^{\prime}=a_{1}$.

Call $I_{1}^{\prime}$ the arc $a_{1}^{\prime}<s<b_{1}^{\prime}$. No normal $\Delta_{s}$ of the arcs $\left(I-I_{1}^{\prime}\right)$ will intersect any of the $\Delta_{s}$ of $I_{1}$.

If $\left(I-I_{1}^{\prime}\right)$ is void we do not proceed any further. In case $\left(\bar{I}-\bar{I}_{1}^{\prime}\right)$ is not void, either (9.4) holds for all $s \in\left(I-I_{1}^{\prime}\right)$, or else there exists a largest open arc $I_{2}: a_{2}<s<b_{2}, I_{2} \subset\left(I-I_{1}^{\prime}\right)$, where (9.3) is true. By Lemma 2 again no two normals $\Delta_{s}$ of $I_{2}$ will intersect. If there are any normals $\Delta_{s}, s \in\left(I-I_{1}^{\prime}\right)$, with $s>b_{2}$ which intersect $\Delta_{b_{2}}$, let $\Delta_{b_{2}^{\prime}}^{\prime}$ be the one for which $b_{2}^{\prime}$ is as large as possible. 
Otherwise let $b_{2}^{\prime}=b_{2}$. Similarly, if there are any $\Delta_{s}, s \in\left(\bar{I}-I_{1}^{\prime}\right)$, with $s<a_{2}$ which intersect $\Delta_{a_{2}}$, let $\Delta_{a_{2}^{\prime}}$ be the one for which $a_{2}^{\prime}$ is as small as possible. Otherwise let $a_{2}^{\prime}=a_{2}$. Call $I_{2}^{\prime}$ the arc $a_{2}^{\prime}<s<b_{2}^{\prime} . I_{1}^{\prime}$ and $I_{2}^{\prime}$ have no points in common, except possibly an end point. Moreover, no normal $\Delta_{s}$, $s \in\left\{\bar{I}-\left(\bar{I}_{1}^{\prime}+\bar{I}_{2}^{\prime}\right)\right\}$, will intersect any of the normals of $\bar{I}_{1}$ and $\bar{I}_{2}$, and none of the normals of $I_{1}$ will intersect any of the normals of $I_{2}$.

Continuing this construction in the manner indicated as long as possible we obtain a finite or infinite sequence of $\operatorname{arcs} I_{n}: a_{n}<s<b_{n}$, in which (9.3) holds, $I_{n}$ being a largest open arc of the set $\left(\bar{I}-\sum_{k=1}^{n-1} \bar{I}_{k}^{\prime}\right)$ for which (9.3) is true. With each $I_{n}$ we obtain an arc $I_{n}^{\prime}: a_{n}^{\prime}<s<b_{n}^{\prime}$ containing $I_{n}$ which is defined in the following manner: If any $\Delta_{s}, s \in\left(\bar{I}-\sum_{k=1}^{n-1} I_{k}^{\prime}\right)$, with $s>b_{n}$ intersects $\Delta_{b_{n}}$, let $\Delta_{b_{n}^{\prime}}$ be the one for which $s=b_{n}^{\prime}$ is a maximum, and if any $\Delta_{s}$, $s \in\left(\bar{I}-\sum_{k=1}^{n-1} I_{k}^{\prime}\right)$, with $s<a_{n}$ intersects $\Delta_{a_{n}}$, let $\Delta_{a_{n}}$ be the one for which $s=a_{n}^{\prime}$ is a minimum. We set $b_{n}^{\prime}=b_{n}$ or $a_{n}^{\prime}=a_{n}$ if there are no such $\Delta_{s}$ for $s>b_{n}$ or $s<a_{n}$ respectively. Then no normal $\Delta_{s}, s \in\left(\bar{I}-\sum_{k=1}^{n} \bar{I}_{k}^{\prime}\right)$, intersects any of the normals $\Delta_{s}$ with $s \in \bar{I}_{k},(k=1,2,3, \cdots, n)$. This implies that the normals of $I_{n}$ and $I_{m}, n>m$, do not intersect. For no $\Delta_{s}, s \in\left(\bar{I}-\sum_{k=1}^{m} \bar{I}_{k}^{\prime}\right)$ intersects any of the $\Delta_{s}, s \in I_{k}(k=1,2, \cdots, m)$, and $I_{n} \subset\left(\bar{I}-\sum_{k=1}^{m} \bar{I}_{k}^{\prime}\right)$, since $n>m$. Moreover, since, for $s \in \bar{I}$, any $\Delta_{s}$ with $s<a_{k}^{\prime}$ cannot intersect any of the $\Delta_{s}$ with $s>b_{k}^{\prime}(k=1,2,3, \cdots)$, it is easily seen that any two $\operatorname{arcs} \bar{I}_{n}^{\prime}$ and $\bar{I}_{m}^{\prime}, n \neq m$ have no points in common except possibly an end point. Hence, the same is true for $\left(\bar{I}_{n}^{\prime}-I_{n}\right)$ and $\left(\bar{I}_{m}^{\prime}-I_{m}\right)$ if $m \neq n$. Let

$$
A=\sum_{k=1}^{\infty} I_{k}, \quad B=\bar{I}-\sum_{k=1}^{\infty} \bar{I}_{k}^{\prime}, \quad C=\sum_{k=1}^{\infty}\left(\bar{I}_{k}^{\prime}-I_{k}\right),
$$

so that $\bar{I}=A+B+C$. By our construction, we have

$$
\text { for } s \in A: \Delta(s) \alpha^{\prime}(s)<1 .
$$

If $B$ is not void, we have

$$
\text { for } s \in B: \Delta(s) \alpha^{\prime}(s) \geqq 1 .
$$

If (9.6) were not true, there would be for at least one

$$
s_{0} \in B: \Delta\left(s_{0}\right) \alpha^{\prime}\left(s_{0}\right)<1 .
$$

Then there exists an open arc $J C I$ containing $s_{0}$ such that (9.3) holds for $s \in J$. We show first that no arc $\bar{I}_{k}^{\prime} \subset J$. Such an arc $I_{k}^{\prime}\left(a_{k}{ }^{\prime}, b_{k}{ }^{\prime}\right)$ must necessarily coincide with its subarc $I_{k}\left(a_{k}, b_{k}\right)$ on which (9.3) holds, for otherwise $\Delta_{a_{k}}$ and $\Delta_{a_{k}^{\prime}}$ or $\Delta_{b_{k}}$ and $\Delta_{b_{k}^{\prime}}$ would intersect and that is impossible by Lemma 2 since $a_{k}^{\prime}, a_{k}, b_{k}, b_{k}^{\prime}$ are all points of $J$, where (9.3) holds. Let $\bar{I}_{n}^{\prime}$ be the first arc in the sequence $\left\{I_{m}{ }^{\prime}\right\}$ (in the order in which the $I_{m}{ }^{\prime}$ were constructed) which is entirely contained in $J$. Since $s_{0}$ is not in any $\bar{I}_{m}^{\prime}, \bar{I}_{n}^{\prime}$ lies either to the right or to the left of $s_{0}$. In either case it follows that, in choosing $I_{n}$, we did 
not select the largest available interval in which (9.3) holds, since in the first case at least the arc $\left(s_{0}, a_{n}\right)$ and in the second case the arc $\left(b_{n}, s_{0}\right)$ should be part of $I_{n}$. Thus, no $\bar{I}_{m}^{\prime}$ can be entirely contained in $J$.

Therefore, there can be in $J$ at most one left end point $a_{\nu}^{\prime}>s_{0}$ of some arc $I_{\nu}^{\prime}$ and one right end point $b_{\mu}^{\prime}<s_{0}$ of some $I_{\mu}^{\prime}$ and the subarc $b_{\mu}^{\prime}<s<a_{\nu}^{\prime}$ of $J$ must remain free of any points belonging to any arcs $I_{m}^{\prime}(m=1,2,3, \cdots)$. But this is impossible: The set of arcs $I_{m}$ is at most denumerable and, if infinite, the lengths of the $I_{m}$ approach 0 as $m \rightarrow \infty$. Since at each step of our construction a largest possible arc in which (9.3) holds is being taken for an $I_{m}$, the arc $b_{\mu}^{\prime}<s<a_{\nu}^{\prime}$ would have to be included at some step in the sequence $\left\{I_{m}\right\}$. If there is no end point $a_{\nu}^{\prime}$ or no end point $b_{\mu}^{\prime}$ in $J$, an even larger subarc of $J$ remains free of points of any $I_{m}^{\prime}$, and this again is impossible. The assumption (9.7) thus leads to a contradiction, and hence (9.6) is true. We obtain therefore

$$
\int_{(B)} \frac{d s}{\Delta(s)} \leqq \int_{(B)} \alpha^{\prime}(s) d s=\int_{(B)}\left|\alpha^{\prime}(s)\right| d s .
$$

Finally, by Lemma 3, on each of the two arcs of $\bar{I}_{m}^{\prime}-I_{m}, a_{m}^{\prime} \leqq s \leqq a_{m}$ and $b_{m} \leqq s \leqq b_{m}{ }^{\prime}$, we have

$$
\begin{aligned}
& \int_{a_{m}^{\prime}}^{a_{m}} \frac{d s}{\Delta(s)} \leqq \frac{1}{\cos ^{3}(2 \epsilon)}\left|\alpha\left(a_{m}\right)-\alpha\left(a_{m}^{\prime}\right)\right|, \\
& \int_{b_{m}}^{b_{m}^{\prime}} \frac{d s}{\Delta(s)} \leqq \frac{1}{\cos ^{3}(2 \epsilon)}\left|\alpha\left(b_{m}^{\prime}\right)-\alpha\left(b_{m}\right)\right| .
\end{aligned}
$$

Hence,

$$
\int_{(C)} \frac{d s}{\Delta(s)} \leqq \frac{1}{\cos ^{3}(2 \epsilon)} \int_{(C)}\left|\alpha^{\prime}(s)\right| d s .
$$

3. Consider now an arc $I_{n}: a_{n}<s<b_{n}$ of $A$. The points on a normal $\Delta_{s}$ are given by the equation

$$
w=w(s)+i t e^{i \alpha(s)}, \quad 0 \leqq t \leqq \Delta(s) .
$$

The integral

$$
\int_{0}^{\Delta(s)}\left|Z^{\prime}\left(w(s)+i t e^{i \alpha(s)}\right)\right| d t \equiv \int_{\Delta_{s}}\left|Z^{\prime}(w)\right| d t \geqq \pi,
$$

since it represents the length of the image of $\Delta_{s}$ in the z-plane. By Schwarz's inequality,

$$
\frac{\pi^{2}}{\Delta(s)} \leqq \int_{\Delta_{s}}\left|Z^{\prime}(w)\right|^{2} d t
$$


If $s \in I_{n}$ and $\alpha^{\prime}(s) \geqq 0$, we have, because of (9.5),

$$
0 \leqq 1-\alpha^{\prime}(s) \Delta(s) \leqq 1-\alpha^{\prime}(s) t \quad \text { for } 0 \leqq t \leqq \Delta(s) ;
$$

and if $\alpha^{\prime}(s)<0$, we have

Hence, in either case

$$
1 \leqq 1-\alpha^{\prime}(s) t \quad \text { for } 0 \leqq t \leqq \Delta(s) \text {. }
$$

$$
-\pi^{2} \int_{a_{n}}^{b_{n}}\left|\alpha^{\prime}(s)\right| d s+\pi^{2} \int_{a_{n}}^{b_{n}} \frac{d s}{\Delta(s)} \leqq \int_{a_{n}}^{b_{n}} d s \int_{0}^{\Delta(s)}\left|Z^{\prime}(w)\right|^{2}\left(1-\alpha^{\prime}(s) t\right) d t .
$$

By what was said in part 2, no.two normals $\Delta_{s}, a_{n} \leqq s \leqq b_{n}$, will intersect, and the transformation (9.10) maps therefore the region $\{0<t<\Delta(s)$, $\left.a_{n}<s<b_{n}\right\}$, of an $(s, t)$-plane in a one-to-one manner onto the (limited) subregion $T_{n}$ of $S$ which is bounded by $C_{-}, C_{+}, \Delta_{a_{n}}$ and $\Delta_{b_{n}}$. The Jacobian of this transformation is $\left(1-\alpha^{\prime}(s) t\right)$. Hence, the last double integral may be written as

Summation over $n$ gives

$$
\iint_{\left(T_{n}\right)}\left|Z^{\prime}(u+i v)\right|^{2} d u d v
$$

(9.11) $-\pi^{2} \int_{(A)}\left|\alpha^{\prime}(s)\right| d s+\pi^{2} \int_{(A)} \frac{d s}{\Delta(s)} \leqq \sum_{n=1}^{\infty} \iint_{\left(T_{n}\right)}\left|Z^{\prime}(u+i v)\right|^{2} d u d v$.

Since, again by the discussion of part 2, the normals $\Delta_{8}$ of any $I_{n}$ do not intersect those of any other $I_{m}(n \neq m)$, the regions $T_{n}$ for $n=1,2,3, \cdots$ do not overlap. Each $T_{n}$ is mapped by $z=Z(w)$ onto a subregion of the strip $|y|<\pi / 2$ whose area is given by $\iint_{\left(T_{n}\right)}\left|Z^{\prime}(u+i v)\right|{ }^{2} d u d v$. Since the $T_{n}$ do not overlap, their images in the $z$-plane do not overlap either, and the total area which these images cover is therefore less than or equal to $\pi\left(x_{2}^{*}-x_{* 1}\right)$. Hence, from

$$
-\pi \int_{(A)}\left|\alpha^{\prime}(s)\right| d s+\pi \int_{(A)} \frac{d s}{\Delta(s)} \leqq x_{2}^{*}-x_{* 1} .
$$

Combining (9.12) with (9.8) and (9.9) we find (9.2).

4. In order to prove now the general case of the theorem (where $\alpha^{\prime}(s)$ is not necessarily continuous) we approximate the $\operatorname{arc} \beta: w=w(s), s_{1} \leqq s \leqq s_{2}$ of $C_{-}$ by a sequence of $\operatorname{arcs} \beta_{n}: w=w_{n}(s), s_{1} \leqq s \leqq s_{2}(n=1,2, \cdots)$, with the following properties $\left({ }^{18}\right)$ :

(i) $w_{n}(s), w_{n}^{\prime}(s), w_{n}^{\prime \prime}(s)$ are continuous for $s_{1} \leqq s \leqq s_{2}, w_{n}\left(s_{k}\right)=w\left(s_{k}\right)$, $w_{n}^{\prime}\left(s_{k}\right)=w^{\prime}\left(s_{k}\right),(k=1,2)$, and $\beta_{n}$ does not intersect any part of the boundary of $S$ except $\beta$.

(18) The functions $w_{n}(s)$ may be found as follows: Let $\alpha(s ; h)=(1 / h) \int_{0}^{s+h} \alpha(t) d t, s \geqq s_{1}, h>0$. Then $\alpha(s ; h) \rightarrow \alpha(s)$ as $h \rightarrow 0$, uniformly for $s_{1} \leqq s \leqq s_{2}$. Let $\alpha(s)=\alpha_{1}(s)-\alpha_{2}(s)$, where $\alpha_{1}(s), \alpha_{2}(s)$ are continuous, non-decreasing functions. The total variation of $\alpha(s ; h)$ in $s_{1} \leqq s \leqq s_{2}$ is 
(ii) $w_{n}(s) \rightarrow w(s)$ and $w_{n}^{\prime}(s) \rightarrow w^{\prime}(s)$ as $n \rightarrow \infty$, uniformly for $s_{1} \leqq s \leqq s_{2}$. This condition implies that if $\sigma=\sigma_{n}(s)$ is the variable arc length of $\beta_{n}$, measured from $s=s_{1}$, and if $\alpha_{n}(s)$ is the angle $\left(\left|\alpha_{n}(s)\right|<\pi / 2\right)$ which the tangent to $\beta_{n}$ at $w_{n}(s)$ forms with the positive real axis, then

$$
\sigma_{n}(s) \rightarrow s-s_{1}, \quad \alpha_{n}(s) \rightarrow \alpha(s)
$$

as $n \rightarrow \infty$,

uniformly for all $s_{1} \leqq s \leqq s_{2}$.

(iii) The total variation

$$
\int_{s=s_{1}}^{s 2}\left|d \alpha_{n}(s)\right| \rightarrow \int_{s-s_{1}}^{s 2}|d \alpha(s)| \quad \text { as } n \rightarrow \infty .
$$

Denote by $S_{n}$ the region obtained from $S$ when the $\operatorname{arc} \beta$ is replaced by $\beta_{n}$. For sufficiently large $n$ and $s \geqq s_{1}, \Delta_{n}(s)$ can be defined for $S_{n}$ as $\Delta(s)$ is defined for $S$ (see part 1 and $\S 8$ (c)). Condition (ii) implies that, as $n \rightarrow \infty$,

$$
\Delta_{n}(s) \rightarrow \Delta(s) \quad \text { uniformly for } s_{1} \leqq s \leqq s_{2} .
$$

By a well known theorem, it follows from (9.13) and (9.15) that

$$
\int_{s-s_{1}}^{s_{2}} \frac{d \sigma_{n}(s)}{\Delta_{n}(s)} \rightarrow \int_{s_{1}}^{s 2} \frac{d s}{\Delta(s)} .
$$

Finally let $S_{n}$ be mapped onto $|y|<\pi / 2$ so that $u=+\infty$ and $x=+\infty$ correspond to each other and that a point $w_{0}$ which is in $S$ and in all $S_{n}$ is carried

Since

$$
\int_{s-s_{1}}^{s_{2}}|d \alpha(s ; h)| \leqq \frac{1}{h} \int_{s-s_{1}}^{s_{2}} d\left[\int_{0}^{o+h} \alpha_{1}(t) d t\right]+\frac{1}{h} \int_{s-s_{1}}^{s_{2}} d\left[\int_{0}^{o+h} \alpha_{2}(t) d t\right] .
$$

$$
\begin{aligned}
\frac{1}{h} \int_{s-s_{1}}^{s_{2}} d\left[\int_{s}^{8+h} \alpha_{i}(t) d t\right] & =\frac{1}{h} \int_{s_{1}}^{s_{2}}\left[\alpha_{i}(s+h)-\alpha_{i}(s)\right] d s=\frac{1}{h} \int_{s_{1}}^{s_{2}} d s \int_{t-s}^{o+h} d \alpha_{i}(t) \\
& \leqq \frac{1}{h} \int_{s-s_{1}}^{s_{2}+h} d \alpha_{i}(t) \int_{t-h}^{t} d s=\int_{s-s_{1}}^{s_{2}+h} d \alpha_{i}(t),
\end{aligned}
$$

we have

$$
\int_{s-s_{1}}^{s_{2}}|d \alpha(s ; h)| \leqq \int_{t-s_{1}}^{s_{2}+h} d \alpha_{1}(t)+\int_{t=s_{1}}^{s_{2}+h} d \alpha_{2}(t) .
$$

Thus, for $h \leqq h_{0}$, the total variation of $\alpha(s ; h)$ in $s_{1} \leqq s \leqq s_{2}$ is uniformly bounded, and there exists, therefore, by a theorem of E. Helly, a sequence $h_{n} \rightarrow 0$ as $n \rightarrow \infty$, such that, for $\bar{\alpha}_{n}(s) \equiv \alpha\left(s ; h_{n}\right)$ :

$$
\int_{s_{1}}^{s_{2}}\left|\bar{\alpha}_{n}^{\prime}(s)\right| d s=\int_{s_{-\infty} s_{1}}^{s_{2}}\left|d \bar{\alpha}_{n}(s)\right| \rightarrow \int_{s_{-\infty} s_{1}}^{s_{2}}|d \alpha(s)| \quad \text { as } n \rightarrow \infty .
$$

Let $w_{1}^{*}=w\left(s_{1}\right), w_{2}^{*}=w\left(s_{2}\right)$. Then we set

$$
w_{n}(s)=w_{1}^{*}+\frac{w_{2}^{*}-w_{1}^{*}}{g_{n}} \int_{s_{1}}^{e} e^{i \alpha_{n}(t)} d t+\left(a_{n} s+b_{n}\right)\left(s-s_{1}\right)\left(s-s_{2}\right),
$$

where $g_{n}=\int_{a_{1}^{2}}^{s^{2}} e^{i \bar{\alpha}_{n}(t)} d t$ and $a_{n}$ and $b_{n}$ are so determined that $w_{n}^{\prime}\left(s_{1}\right)=w^{\prime}\left(s_{1}\right), w_{n}^{\prime}\left(s_{2}\right)=w^{\prime}\left(s_{2}\right)$. It is easily seen that $\lim _{n \rightarrow+\infty} g_{n}=w_{2}^{*}-w_{1}^{*}, \lim _{n \rightarrow \infty} a_{n}=\lim _{n \rightarrow \infty} b_{n}=0$ and that the $w_{n}(s)$ satisfy the three conditions (i), (ii), (iii) of the text. 
into $Z\left(w_{0}\right)$. Let $x_{* 1}^{(n)}$ and $x_{2}^{*(n)}$ be defined for $S_{n}$ as $x_{* 1}$ and $x_{2}^{*}$ are for $S$. Then( ${ }^{(19)}$

$$
\lim _{n \rightarrow \infty} x_{* 1}^{(n)}=x_{* 1} \text { and } \lim _{n \rightarrow \infty} x_{2}^{*(n)}=x_{2}^{*} \text {. }
$$

For sufficiently large $n, S_{n}$ will satisfy the condition stated at the end of part 1 of this proof, since $S$ satisfies it. Moreover, since $\beta_{n}$ has continuous curvature, we have by $(9.2)$

$$
-\frac{\pi}{\cos ^{3}(2 \epsilon)} \int_{s=s_{1}}^{s_{2}}\left|d \alpha_{n}(s)\right|+\pi \int_{s_{1}}^{s_{2}} \frac{d \sigma_{n}(s)}{\Delta_{n}(s)} \leqq x_{2}^{*(n)}-x_{* 1}^{(n)} .
$$

Letting $n \rightarrow \infty$ we find because of (9.14), (9.16), and (9.17),

$$
-\frac{\pi}{\cos ^{3}(2 \epsilon)} \int_{s=s_{1}}^{s_{2}}|d \alpha(s)|+\pi \int_{s_{1}}^{s_{2}} \frac{d s}{\Delta(s)} \leqq x_{2}^{*}-x_{* 1} .
$$

Finally, to prove (9.1) we need only observe that by Lemma $1 x_{2}^{*}-x_{2} \rightarrow 0$ and $x_{1}-x_{* 1} \rightarrow 0$ as $s_{1} \rightarrow \infty$ and that $\epsilon$ may be taken arbitrarily small, provided $s_{1}$ is chosen sufficiently large. This completes the proof of Theorem V.

10. A definition and further lemmas. Theorem $\mathrm{V}$ will enable us to obtain a lower bound for the difference $x_{2}-x_{1}$ of the type mentioned in $\$ 7$. We introduce first the following definition.

DEFINITION. Let $S$ be an L-strip in the w-plane with the boundary inclination $\gamma,|\gamma|<\pi / 2$, at $u=+\infty$. Let $v=\phi_{+}(u)$ and $v=\phi_{-}(u)$ represent the boundaary curves $C_{+}$and $C_{-}$of $S$, respectively. We shall say that $S$ is a strip with finite boundary turning at $u=+\infty$ if $\phi_{+}^{\prime}(u)$ and $\phi_{-}^{\prime}(u)$ are continuous for all suffciently large $u$, say $u \geqq u_{1}$, and if the integrals

converge.

$$
\int_{u=u_{1}}^{\infty}\left|d \phi_{+}^{\prime}(u)\right|, \quad \int_{u=u_{1}}^{\infty}\left|d \phi_{-}^{\prime}(u)\right|
$$

Our object will be accomplished by the following lemma.

Lemma 4. Let $S$ be an $L$-strip in the w-plane with the boundary inclination $\gamma$, $|\gamma|<\pi / 2$, and with finite boundary turning at $u=+\infty$. If $\Delta(s)$ and $w(s)$ are defined as in $\S 8$, then for $u=R w(s)$ and $s_{1}<s_{2}\left(u_{1}=R w\left(s_{1}\right), u_{2}=R w\left(s_{2}\right)\right)$

$$
\text { 1) } \int_{s_{1}}^{s_{2}} \frac{d s}{\Delta(s)}=\int_{u_{1}}^{u_{2}} \frac{1+\psi^{\prime 2}(u)}{\theta(u)} d u-\frac{1}{4} \int_{u_{1}}^{u_{2}} \frac{\theta^{\prime 2}(u)}{\theta(u)} d u+o(1) \text { as } s_{1} \rightarrow \infty \text {. }
$$

Proof. Denote the point $w(s)$ on $C_{-}$by $A$, the other end point of $\Delta_{s}$ by $B$ and the point $u+i \phi_{+}(u)$, where $u=R w(s)$, by $C$. Suppose, for the present, that $B$ lies in the half-plane to the left of $A C$. In the triangle $A B C$ the angle

$\left({ }^{19}\right)$ This follows from the theorem of Rado used in the proof of (6.9); see (12). 
$A=\alpha=\alpha(s)$. To find the angle $C$, observe that by the mean value theorem there exists a point $w^{*}=u^{*}+i \phi_{+}\left(u^{*}\right)$ on the arc $B C$ of $C_{+}$such that the tangent to $C_{+}$at $w^{*}$ is parallel to the chord $B C$. If the angle of inclination of this tangent is $\beta^{*},\left|\beta^{*}\right|<\pi / 2$, then the angle $C=\pi / 2-\beta^{*}$. Let $\beta,|\beta|<\pi / 2$, denote the angle of inclination of the tangent to $C_{+}$at $C$. By the law of sines we obtain from the triangle $A B C$ :

(10.2) $\frac{A C}{A B}=\frac{\theta(u)}{\Delta(s)}=\frac{\sin (A+C)}{\sin C}=\frac{\cos \left(\beta^{*}-\alpha\right)}{\cos \beta^{*}}=\frac{\cos \left[(\beta-\alpha)+\left(\beta^{*}-\beta\right)\right]}{\cos \left[\beta+\left(\beta^{*}-\beta\right)\right]}$.

Since $\alpha, \beta, \beta^{*}$ approach $\gamma$ as $s \rightarrow \infty$ and since $|\gamma|<\pi / 2$, we may write (10.2) in the form

$$
\frac{\theta(u)}{\Delta(s)}=\frac{\cos (\beta-\alpha)}{\cos \beta}\left(1+O\left(\left|\beta^{*}-\beta\right|\right)\right)
$$

Now

Moreover,

$$
\left|\beta^{*}-\beta\right| \leqq\left|\tan \beta^{*}-\tan \beta\right| \leqq\left|\int_{t=u}^{u^{*}}\right| d \phi_{+}^{\prime}(t)||
$$

$$
\left|u-u^{*}\right| \leqq B C=\frac{\sin A}{\sin B} \theta(u)<\theta(u)
$$

for sufficiently large $u$, since $B \rightarrow \pi / 2$ and $A \rightarrow|\gamma|$ as $u \rightarrow+\infty$. Hence

$$
\left|\beta^{*}-\beta\right| \leqq \Phi_{+}(u) \equiv \int_{t=u-\theta(u)}^{u+\theta(u)}\left|d \phi_{+}^{\prime}(t)\right| .
$$

The quotient

$$
\frac{\cos (\beta-\alpha)}{\cos \beta \cos \alpha}=1+\tan \alpha \tan \beta=1+\phi_{+}^{\prime}(u) \phi_{-}^{\prime}(u)=1+{\psi^{\prime}}^{\prime 2}(u)-\left[\frac{\theta^{\prime}(u)}{2}\right]^{2},
$$

so that

$$
\frac{1}{\Delta(s)}=\frac{\cos \alpha}{\theta(u)}\left(1+\psi^{\prime 2}(u)\right)-\frac{\cos \alpha}{\theta(u)}\left[\frac{\theta^{\prime}(u)}{2}\right]^{2}+O\left(\frac{\Phi_{+}(u)}{\theta(u)} \cos \alpha\right)
$$

as $u \rightarrow+\infty$. This relation is also easily verified if $B$ lies to the right of $A C$. Observing now that $\cos \alpha \cdot d s=d u$, we obtain

$$
\int_{s_{1}}^{s_{2}} \frac{d s}{\Delta(s)}=\int_{u_{1}}^{u_{2}} \frac{1+\psi^{\prime 2}(u)}{\theta(u)} d u-\frac{1}{4} \int_{u_{1}}^{u_{2}} \frac{\theta^{\prime 2}(u)}{\theta(u)} d u+O\left\{\int_{u_{1}}^{u_{2}} \frac{\Phi_{+}(u)}{\theta(u)} d u\right\} .
$$

The result (10.1) follows now immediately from

Lemma 5. Under the hypotheses of Lemma 4 the integral 


$$
\int^{\infty} \frac{\Phi_{+}(u)}{\theta(u)} d u \quad \text { converges, } \quad \Phi_{+}(u) \equiv \int_{t=u-\theta(u)}^{u+\theta(u)}\left|d \phi_{+}^{\prime}(t)\right| .
$$

Proof. We have for $b<c$ :

$$
\begin{aligned}
I \equiv \int_{b}^{c} \frac{\Phi_{+}(u)}{\theta(u)} d u= & \int_{b}^{c} \frac{1}{\theta(u)}\left[\int_{t=u}^{u+\theta(u)}\left|d \phi_{+}^{\prime}(t)\right|\right] d u \\
& +\int_{b}^{c} \frac{1}{\theta(u)}\left[\int_{t=u-\theta(u)}^{u}\left|d \phi_{+}^{\prime}(t)\right|\right] d u=I_{1}+I_{2} .
\end{aligned}
$$

Interchanging of the order of integration in $I_{1}$ gives

$$
\begin{aligned}
I_{1} & =\int_{t=b}^{c}\left|d \phi_{+}^{\prime}(t)\right| \int_{o(t)}^{t} \frac{d u}{\theta(u)}+\int_{t=e}^{c+\theta(c)}\left|d \phi_{+}^{\prime}(t)\right| \int_{o(t)}^{c} \frac{d u}{\theta(u)} \\
& \leqq \int_{t=b}^{c+\theta(c)}\left|d \phi_{+}^{\prime}(t)\right| \int_{o(t)}^{t} \frac{d u}{\theta(u)},
\end{aligned}
$$

where $u=g(t)$ is the inverse function $\left({ }^{20}\right)$ of $t=u+\theta(u)$ for $b+\theta(b) \leqq t \leqq c+\theta(c)$, $u=g(t) \equiv b$ for $b \leqq t \leqq b+\theta(b)$. Now $0 \leqq t-g(t) \leqq \theta(g(t))$ for $b \leqq t \leqq c+\theta(c)$. Hence, by Lemma 7 (which is proved in $\S 15$ ),

$$
\theta(u) \geqq \frac{1}{2} \theta(g(t))
$$

for $g(t) \leqq u \leqq t$,

provided only $b$ is sufficiently large. Thus

$$
\int_{o(t)}^{t} d u / \theta(u) \leqq 2[(t-g(t)) / \theta(g(t))] \leqq 2,
$$

and hence

$$
I_{1} \leqq 2 \int_{t=b}^{c+\theta(c)}\left|d \phi_{+}^{\prime}(t)\right|
$$

Similarly,

$$
I_{2} \leqq 2 \int_{t=b-\phi(b)}^{c}\left|d \phi_{+}^{\prime}(t)\right|,
$$

if $b$ is sufficiently large, so that

$$
I \leqq 4 \int_{t=b-\theta(b)}^{c+\theta(c)}\left|d \phi_{+}^{\prime}(t)\right| .
$$

Hence (10.4) follows from the hypothesis that $S$ has finite boundary turning at $u=+\infty$.

11. The second basic inequality. We are now in the position to prove our second basic inequality.

(20) The inverse function of $t=u+\theta(u)$ exists if $b$ is sufficiently large, since $d t / d u=1+\theta^{\prime}(u)$, and therefore approaches 1 as $u \rightarrow+\infty$. 
THEOREM VI. Let $S$ be an L-strip in the w-plane with the boundary inclination $\gamma=0$ and with finite boundary turning at $u=+\infty$. Suppose that $z=Z(w)=X(w)+i Y(w)$ maps $S$ conformally onto the strip $|y|<\pi / 2$ and that $\lim _{u \rightarrow+\infty} X(w)=+\infty$. Let $w_{1}=u_{1}+i v_{1}, w_{2}=u_{2}+i v_{2}, u_{1}<u_{2}$, be two points in $\bar{S}$ and let $x_{1}=X\left(w_{1}\right), x_{2}=X\left(w_{2}\right)$. Then

$$
x_{2}-x_{1} \geqq \pi \int_{u_{1}}^{u_{2}} \frac{1+\psi^{\prime 2}(u)}{\theta(u)} d u-\frac{\pi}{4} \int_{u_{1}}^{u_{2}} \frac{\theta^{\prime 2}(u)}{\theta(u)} d u+o(1)
$$

as $u_{1}$ and hence $u_{2}$ approach $+\infty$, uniformly in $v_{1}$ and $v_{2}$.

Proof. Let $w(s)$ be defined as in $\S 8(\mathrm{~b})$, and let $R w\left(s_{k}\right)=u_{k}, X\left(u_{k}+i \phi_{-}\left(u_{k}\right)\right)$ $=x_{k}^{\prime} \quad(k=1,2)$. By Theorem $\mathrm{V}$ we have, since $\int_{t=u_{1}}^{\infty}\left|d \phi_{-}^{\prime}(t)\right|<\infty$,

$$
x_{2}^{\prime}-x_{1}^{\prime} \geqq \pi \int_{s_{1}}^{s_{2}} \frac{d s}{\Delta(s)}+o(1) \text { as } s_{1} \text { and hence } s_{2} \rightarrow \infty \text {. }
$$

By Lemma $1, \lim _{u_{2} \rightarrow+\infty}\left(x_{2}-x_{2}^{\prime}\right)=0$ and $\lim _{u_{1} \rightarrow+\infty}\left(x_{1}-x_{1}^{\prime}\right)=0$ so that the difference $x_{2}^{\prime}-x_{1}^{\prime}$ in (11.2) may be replaced by $x_{2}-x_{1}+o(1)$. The result (11.1) follows now immediately from Lemma 4.

\section{A DISTORTION THEOREM}

12. A lemma. If in Theorems IV and VI it is assumed that the integral

$$
\int^{\infty} \frac{\theta^{\prime 2}(u)}{\theta(u)} d u \text { converges, }
$$

then their combination yields an asymptotic expression for $x_{2}-x_{1}$,

$$
x_{2}-x_{1}=\int_{u_{1}}^{u_{2}} \frac{1+\psi^{\prime 2}(u)}{\theta(u)} d u+o(1) \text { as } u_{1} \text { and } u_{2} \rightarrow+\infty .
$$

One of the hypotheses under which this formula is obtained is that the boundary inclination of $S$ at $u=+\infty$ is $\gamma=0$. It is easy to modify it so as to obtain a result which holds for any $\gamma,|\gamma|<\pi / 2$. For this purpose we prove first the following lemma.

Lemma 6. Let $S$ be an $L$-strip in the w-plane $(w=u+i v)$ with the boundary inclination $\gamma,|\gamma|<\pi / 2$, and with finite boundary turning at $u=+\infty$. Let $v=\phi_{+}(u)$ and $v=\phi_{-}(u)$ represent its boundary curves $C_{+}$and $C_{-}$, respectively. Suppose that a new set of coordinates $\bar{u}, \bar{v}(\bar{u}+i \bar{v}=\bar{w})$ is introduced by means of the rotation $\bar{w}=w e^{-i \gamma}$ and that, for sufficiently large $\bar{u}, C_{+}$and $C_{-}$are represented in the new coordinate system by $\bar{v}=\bar{\phi}_{+}(\bar{u})$ and $\bar{v}=\bar{\phi}_{-}(\bar{u})$, respectively. Set

$$
\theta(u)=\phi_{+}(u)-\phi_{-}(u), \quad \bar{\theta}(\bar{u})=\bar{\phi}_{+}(\bar{u})-\bar{\phi}_{-}(\bar{u}) .
$$

If $u$ and $\bar{u}$ are connected by the relation 


$$
\bar{u}=u \cos \gamma+\phi_{-}(u) \sin \gamma,
$$

then, for sufficiently large $u$,

$$
\left|\bar{\theta}^{\prime}(\bar{u})\right| \leqq 2\left\{\left|\theta^{\prime}(u)\right|+\Phi_{+}(u)\right\}, \quad \Phi_{+}(u) \equiv \int_{t=u-\theta(u)}^{u+\theta(u)}\left|d \phi_{+}^{\prime}(t)\right| .
$$

Thus, the convergence of the integral

implies that of

$$
\int^{\infty} \frac{\theta^{\prime 2}(u)}{\theta(u)} d u
$$

(by (8.1) and Lemma 5).

$$
\int^{\infty} \frac{\bar{\theta}^{\prime 2}(\bar{u})}{\bar{\theta}(\bar{u})} d \bar{u}
$$

Proof. Let $u$ and $\bar{u}$ satisfy (12.2). We denote the points $\bar{u}+i \bar{\phi}_{-}(\bar{u})$ and $\bar{u}+i \bar{\phi}_{+}(\bar{u})$ of the $\bar{v}$-plane by $A$ and $B$, respectively. The coordinates of $A$ and $B$ in the $w$-plane are then $u+i \phi_{-}(u)$ and $u_{1}+i \phi_{+}\left(u_{1}\right)$, respectively (where $u_{1}$ is determined by the relation $\left.e^{-i \gamma}\left(u_{1}+i \phi_{+}\left(u_{1}\right)\right)=\bar{u}+i \bar{\phi}_{+}(\bar{u})\right)$. Finally, we call $C$ the point $u+i \phi_{+}(u)$ of the $w$-plane. In the triangle $A B C, A B=\bar{\theta}(\bar{u})$, $A C=\theta(u)$ and the angle $A=|\gamma|$.

Let $\alpha_{+}(u)$ and $\alpha_{-}(u)$ denote in the $w$-plane the angles of inclination of the tangents to $C_{+}$and to $C_{-}$, respectively, at a point with the abscissa $u$. Then

$$
\begin{array}{ll}
\phi_{-}^{\prime}(u)=\tan \alpha_{-}(u), & \phi_{+}^{\prime}(u)=\tan \alpha_{+}(u) ; \\
\bar{\phi}_{-}^{\prime}(\bar{u})=\tan \left\{\alpha_{-}(u)-\gamma\right\}, & \bar{\phi}_{+}^{\prime}(\bar{u})=\tan \left\{\alpha_{+}\left(u_{1}\right)-\gamma\right\} .
\end{array}
$$

Hence,

$$
\bar{\theta}^{\prime}(\bar{u})=\tan \left\{\alpha_{+}\left(u_{1}\right)-\alpha_{-}(u)\right\} \cdot\left\{1+\bar{\phi}_{-}^{\prime}(\bar{u}) \bar{\phi}_{+}^{\prime}(\bar{u})\right\} .
$$

Since $\bar{\phi}_{-}^{\prime}(\bar{u})$ and $\bar{\phi}_{+}^{\prime}(\bar{u})$ approach 0 as $\bar{u} \rightarrow+\infty$, we may write:

$$
\left|\bar{\theta}^{\prime}(\bar{u})\right| \leqq\left\{\left|\theta^{\prime}(u)\right|+\left|\phi_{+}^{\prime}\left(u_{1}\right)-\phi_{+}^{\prime}(u)\right|\right\}(1+o(1))
$$

as $u \rightarrow+\infty$. Now

$$
\left|u_{1}-u\right| \leqq B C=\frac{\sin A}{\sin B} \theta(u) \leqq \theta(u)
$$

for sufficiently large $u$, since the angle $B \rightarrow \pi / 2$ and $A=|\gamma|<\pi / 2$. Hence, $\left|\phi_{+}^{\prime}\left(u_{1}\right)-\phi_{+}^{\prime}(u)\right| \leqq\left|\int_{t-u}^{u_{1}}\right| d \phi_{+}^{\prime}(t)|| \leqq \Phi_{+}(u)$, and (12.3) follows from (12.4).

13. First form of the distortion theorem. We prove now

ThEOREM VII. Let $S$ be an L-strip with the boundary inclination $\gamma$, $|\gamma|<\pi / 2$ and with finite boundary turning at $u=+\infty$. Moreover let the integral 


$$
\int^{\infty} \frac{\theta^{\prime 2}(u)}{\theta(u)} d u \text { be convergent }\left({ }^{21}\right) \text {. }
$$

Suppose that $z=Z(w)=X(w)+i Y(w)$ maps $S$ conformally onto the strip $|y|<\pi / 2$ in such a manner that $\lim _{u \rightarrow+\infty} X(w)=+\infty$. Let $\Delta_{s}, \Delta(s)$ and $w(s)$ be defined as in \$8. Then, if $s_{1}<s_{2}, w_{1} \in \Delta_{s_{1}}, w_{2} \in \Delta_{s_{2}}$,

$$
X\left(w_{2}\right)-X\left(w_{1}\right)=\pi \int_{s_{1}}^{s_{2}} \frac{d s}{\Delta(s)}+o(1) \quad \text { as } s_{1} \text { and } s_{2} \rightarrow \infty,
$$

uniformly with respect to $w_{1}$ and $w_{2}$.

Proof. As in Lemma 6, let there be introduced a new set of axes $(\bar{u}, \bar{v})$, $\bar{u}+i \bar{v}=\bar{w}$, by means of the rotation $\bar{w}=e^{-i \gamma} w$. Let $\bar{\phi}_{+}(u), \bar{\phi}_{-}(u), \bar{\theta}(\bar{u})$ be defined as in Lemma 6 and let $u$ and $\bar{u}$ be two numbers related by equation (12.2). Then, by Lemma 6, (13.1) implies that

$$
\int^{\infty} \frac{\bar{\theta}^{\prime 2}(\bar{u})}{\bar{\theta}(\bar{u})} d \bar{u}
$$

converges. Let $\bar{u}_{1}$ and $\bar{u}_{2}$ be the abscissas of the points $w\left(s_{1}\right)$ and $w\left(s_{2}\right)$ (on $C_{-}$) in the $(\bar{u}, \bar{v})$-system, and let $x_{1}^{\prime}=X\left(w\left(s_{1}\right)\right), \quad x_{2}^{\prime}=X\left(w\left(s_{2}\right)\right)$. If $\bar{\psi}(\bar{u})$ $=\frac{1}{2}\left[\bar{\phi}_{+}(\bar{u})+\bar{\phi}_{-}(\bar{u})\right]$, we have by Theorem IV,

$$
x_{2}^{\prime}-x_{1}^{\prime} \leqq \pi \int_{\bar{u}_{1}}^{\bar{u}_{2}} \frac{1+\bar{\psi}^{\prime 2}(\bar{u})}{\bar{\theta}(\bar{u})} d \bar{u}+\frac{\pi}{12} \int_{\bar{u}_{1}}^{\bar{u}_{2}} \frac{\bar{\theta}^{\prime 2}(\bar{u})}{\bar{\theta}(\bar{u})} d \bar{u}+o(1),
$$

as $s_{1}$ and $s_{2} \rightarrow \infty$. By Lemma 4 and by (13.3) we obtain

$$
x_{2}^{\prime}-x_{1}^{\prime} \leqq \int_{s_{1}}^{s_{2}} \frac{d s}{\Delta(s)}+o(1) \quad \text { as } s_{1}, s_{2} \rightarrow \infty .
$$

Finally, application of Lemma 1 shows that $\lim _{s_{1} \rightarrow \infty}\left(x_{1}-x_{1}^{\prime}\right)=\lim _{s_{1} \rightarrow \infty}\left(x_{2}-x_{2}^{\prime}\right)$ $=0\left(x_{k}=X\left(w_{k}\right)\right)$, so that the difference $x_{2}^{\prime}-x_{1}^{\prime}$ in (13.4) may be replaced by $x_{2}-x_{1}$. Combining this result with Theorem V, we obtain (13.2).

14. Second form of the distortion theorem. For some applications the following form of Theorem VII will be more convenient.

THEOREM VIII. Let $S$ be an L-strip satisfying all the hypotheses of Theorem VII, and let $z=Z(w)$ be defined as in that theorem. Then for $w_{1}=u_{1}+i v_{1}$ and $w_{2}=u_{2}+i v_{2}$ in $\bar{S}, u_{1}<u_{2}$,

(21) Condition (13.1) is automatically satisfied for $L$-strips with finite boundary turning at $u=+\infty$ for which $0<c_{1} \leqq \theta(u) \leqq c_{2}\left(c_{1}, c_{2}\right.$ constants). For

$$
\int_{a}^{b} \frac{\theta^{\prime 2}(u)}{\theta(u)} d u=\left.\theta^{\prime}(u) \log \theta(u)\right|_{a} ^{b}-\int_{u=a}^{b} \log \theta(u) d \theta^{\prime}(u) .
$$

Since $|\log \theta(u)|$ is bounded and $\int_{u=a}^{\infty}\left|d \theta^{r}(u)\right|$ exists, (13.1) follows. 


$$
\begin{aligned}
X\left(w_{2}\right)-X\left(w_{1}\right)=\pi & \int_{u_{1}}^{u_{2}} \frac{1+\psi^{\prime 2}(u)}{\theta(u)} d u \\
& +\pi\left[\frac{v_{2}-\psi\left(u_{2}\right)}{\theta\left(u_{2}\right)}-\frac{v_{1}-\psi\left(u_{1}\right)}{\theta\left(u_{1}\right)}\right] \tan \gamma+o(1),
\end{aligned}
$$

as $u_{1}$ and $u_{2} \rightarrow+\infty$, uniformly with respect to $v_{1}$ and $v_{2}$.

Proof. Let $\Delta_{s}, \Delta(s)$ and $w(s)$ be defined as in $\S 8$. Let $\Delta_{s_{1}}$ and $\Delta_{s_{2}}$ be two normals of $C_{-}$which pass through $w_{1}$ and $w_{2}$, respectively. By Theorem VII,

$$
X\left(w_{2}\right)-X\left(w_{1}\right)=\pi \int_{s_{1}}^{s_{2}} \frac{d s}{\Delta(s)}+o(1) \quad \text { as } s_{1}, s_{2} \rightarrow \infty .
$$

Let $R w\left(s_{1}\right)=u_{1}^{\prime}, R w\left(s_{2}\right)=u_{2}^{\prime}$. By Lemma 4 and (13.1) this equals

$$
\pi \int_{u^{\prime}}^{u^{\prime}} \frac{1+\psi^{\prime 2}(u)}{\theta(u)} d u+o(1), \quad \text { as } u_{1}, u_{2} \rightarrow+\infty,
$$

or

$$
X\left(w_{2}\right)-X\left(w_{1}\right)=\pi \int_{u_{1}^{\prime}}^{u_{1}}+\pi \int_{u_{1}}^{u_{2}}+\pi \int_{u_{2}}^{u_{2}^{\prime}}+o(1) .
$$

We estimate the first and third of the integrals in (14.1). Assume, for the present, that $\gamma>0$. Denote the points $u_{1}+i \phi_{-}\left(u_{1}\right), w\left(s_{1}\right)$ and $w_{1}$ by $A, B$, and $C$, respectively. In the triangle $A B C$ the side $A C=v_{1}-\phi_{-}\left(u_{1}\right)$, the angle $C \rightarrow \gamma$ and the angle $B \rightarrow \pi / 2$ as $u_{1} \rightarrow+\infty$. These limits, as well as all following in this proof (taken as $u_{1} \rightarrow+\infty$ ) exist uniformly with respect to the position of $w_{1}$ on $\theta_{u_{1}}$. Now

$$
\begin{aligned}
u_{1}^{\prime}-u_{1} & =B C \sin \left(\gamma+\epsilon_{1}\right), & \lim _{u_{1} \rightarrow+\infty} \epsilon_{1} & =0, \\
B C=A C \frac{\sin A}{\sin B} & =A C \frac{\cos \left(\gamma+\epsilon_{2}\right)}{\sin B}, & \lim _{u_{1} \rightarrow+\infty} \epsilon_{2} & =0,
\end{aligned}
$$

and hence

$$
u_{1}^{\prime}-u_{1}=\left[v_{1}-\phi_{-}\left(u_{1}\right)\right] \sin \left(\gamma+\epsilon_{1}\right) \cos \left(\gamma+\epsilon_{2}\right) \frac{1}{\sin B} .
$$

This result is also easily verified when $\gamma \leqq 0$.

Now, by the law of the mean

$$
\int_{u_{1}}^{u_{1}^{\prime}} \frac{1+\psi^{\prime 2}(u)}{\theta(u)} d u=\left(u_{1}^{\prime}-u_{1}\right) \frac{1+\psi^{\prime 2}\left(u^{*}\right)}{\theta\left(u^{*}\right)} \quad\left(u^{*} \text { between } u_{1} \text { and } u_{1}^{\prime}\right) .
$$

Since, by (14.2), $\left|u_{1}^{\prime}-u_{1}\right| \leqq \theta\left(u_{1}\right)$ for sufficiently large $u_{1}$, it follows from Lemma 7 (which is proved in $\$ 15$ ) that $\left(\theta\left(u^{*}\right) / \theta\left(u_{1}\right)\right) \rightarrow 1$ as $u_{1} \rightarrow+\infty$. Furthermore, as $u_{1} \rightarrow+\infty, 1+\psi^{\prime 2}\left(u^{*}\right) \rightarrow 1 / \cos ^{2} \gamma$. Hence 


$$
\begin{aligned}
\int_{u_{1}}^{u^{\prime}} \frac{1+\psi^{\prime 2}(u)}{\theta(u)} d u & =\frac{v_{1}-\phi_{-}\left(u_{1}\right)}{\theta\left(u_{1}\right)} \tan \gamma+o(1) \\
& =\left[\frac{v_{1}-\psi\left(u_{1}\right)}{\theta\left(u_{1}\right)}+\frac{1}{2}\right] \tan \gamma+o(1)
\end{aligned}
$$

as $u_{1} \rightarrow+\infty$. An analogous result is obtained for the third integral in (14.1). Substitution of these expressions into (14.1) proves the theorem.

CoROLlaRY OF THEOREM VIII. Under the hypotheses of the theorem, for $w=u+i v \in \bar{S}$,

$$
\lim _{u \rightarrow+\infty}\left\{X(w)-\left[\pi \int_{u_{0}}^{u} \frac{1+\psi^{\prime 2}(t)}{\theta(t)} d t+\pi \frac{v-\psi(u)}{\theta(u)} \tan \gamma\right]\right\}=\lambda
$$

exists uniformly in $v$ and is finite.

For, if the difference within the braces in (14.3) is denoted by $A(w)$, Theorem VIII states that for any given $\epsilon>0$ there exists an $N(\epsilon)$ such that for all $w_{1}$ and $w_{2}$ in $S$ for which $R w_{2}>R w_{1}>N(\epsilon)$ :

$$
\left|A\left(w_{2}\right)-A\left(w_{1}\right)\right|<\epsilon .
$$

THEOREM IX. Let S be an L-strip satisfying the hypotheses of Theorem VII and let $z=Z(w)$ be defined as in that theorem. Then, for $w=u+i v \in \bar{S}$,

$$
Z(w)=\lambda+\pi \int_{u_{0}}^{u} \frac{1+\psi^{\prime 2}(t)}{\theta(t)} d t+\pi \frac{v-\psi(u)}{\theta(u)} \tan \gamma+i \pi \frac{v-\psi(u)}{\theta(u)}+o(1),
$$

as $u \rightarrow+\infty$, uniformly in $v . \lambda$ is a real constant.

This result follows immediately by combining (14.3) with the result (18.1) of Corollary 1 of Theorem X (which is proved in \$18).

\section{ASYMPTOTIC BEHAVIOR OF THE MAPPING FUNCTION OF AN $L$-STRIP AND OF ITS DERIVATIVE}

15. Preliminary remarks and lemmas. We shall establish now asymptotic expressions for $Z(w)$ and $Z^{\prime}(w)$ under the mere assumption that $S$ is an $L$-strip. These results will be less sharp than those of Part IV. While in Part IV (Theorem IX) we obtained, under more restrictive assumptions, an expression $f(w)$ such that the difference $Z(w)-f(w)$ approaches a finite limit, as $u \rightarrow+\infty$, we shall find here expressions for $Z(w)$ and $Z^{\prime}(w)$ which represent these functions merely in the sense of asymptotic equivalence (that is, the quotient of the function in question and its asymptotic expression approaches 1 as $u \rightarrow+\infty)$. None of the results of Parts III and IV will be used here.

Throughout this part we assume $S$ to be an $L$-strip in the $w$-plane with the boundary inclination $\gamma,|\gamma|<\pi / 2$ at $u=+\infty$, and $Z(w)=X(w)+i Y(w)$ 
a function which maps $S$ conformally onto the strip $|y|<\pi / 2$ in such a manner that $\lim _{u \rightarrow+\infty} X(w)=+\infty$. The inverse of $z=Z(w)$ will be denoted by $w=W(z)=U(z)+i V(z)$.

We shall make use of the following simple lemma.

LEMMA 7. Let, for $u_{0}<u_{1}<u_{2}$,

and let

$$
\theta^{*}\left(u_{1}, u_{2}\right)=\max _{u_{1} \leqq u \leqq u_{2}} \theta(u), \quad \theta_{*}\left(u_{1}, u_{2}\right)=\min _{u_{1} \leqq u \leqq u_{2}} \theta(u)
$$

$$
u_{2}-u_{1} \leqq k \theta^{*}\left(u_{1}, u_{2}\right),
$$

$k$ a constant.

Then, uniformly for all $u_{2}$,

$$
\lim _{u_{1} \rightarrow+\infty} \frac{\theta_{*}\left(u_{1}, u_{2}\right)}{\theta^{*}\left(u_{1}, u_{2}\right)}=1
$$

Proof. Let $\theta^{*}\left(u_{1}, u_{2}\right)=\theta(b)$ and $\theta_{*}\left(u_{1}, u_{2}\right)=\theta(c), u_{1} \leqq b, c \leqq u_{2}$. If $u_{1}$ is suffiiently large, we have

$$
|\theta(b)-\theta(c)|=\left|\int_{c}^{b} \theta^{\prime}(u) d u\right| \leqq \int_{u_{1}}^{u_{2}}\left|\theta^{\prime}(u)\right| d u \leqq\left(u_{2}-u_{1}\right) \sup _{u_{1} \leqq u \leqq u_{2}}\left|\theta^{\prime}(u)\right|,
$$

(where $\sup _{u_{1} \leqq u \leqq u_{2}}\left|\theta^{\prime}(u)\right|$ denotes the least upper bound of $\left|\theta^{\prime}(u)\right|$ in $\left.u_{1} \leqq u \leqq u_{2}\right)$. Hence by use of (15.1):

$$
0 \leqq 1-\frac{\theta_{*}\left(u_{1}, u_{2}\right)}{\theta^{*}\left(u_{1}, u_{2}\right)} \leqq \frac{u_{2}-u_{1}}{\theta^{*}\left(u_{1}, u_{2}\right)} \sup _{u_{1} \leqq u \leqq u_{2}}\left|\theta^{\prime}(u)\right| \leqq k \sup _{u_{1} \leqq u \leqq u_{2}}\left|\theta^{\prime}(u)\right| \rightarrow 0,
$$

as $u_{1} \rightarrow+\infty$, by hypothesis. This proves the lemma.

COROLlary. Let

$$
0 \leqq x_{2}-x_{1} \leqq c, \quad c>0, a \text { coristant }
$$

and $u_{1}=\min _{|y| \leqq \pi / 2} U\left(x_{1}+i y\right), u_{2}=\max _{|y| \leqq \pi / 2} U\left(x_{2}+i y\right)$. Then, uniformly for all $x_{2}$, satisfying (15.2),

$$
\lim _{x_{1} \rightarrow+\infty} \frac{\theta^{*}\left(u_{1}, u_{2}\right)}{\theta_{*}\left(u_{1}, u_{2}\right)}=1 .
$$

Proof. From Theorem III (a) we have

$$
\pi \int_{u_{1}}^{u_{2}} \frac{d u}{\theta(u)} \leqq x_{2}-x_{1}+4 \pi \leqq c+4 \pi
$$

by (15.2). On the other hand, since $u_{2} \geqq u_{1}$,

$$
\int_{u_{1}}^{u_{2}} \frac{d u}{\theta(u)} \geqq \frac{u_{2}-u_{1}}{\theta^{*}\left(u_{1}, u_{2}\right)},
$$


and hence $0 \leqq u_{2}-u_{1} \leqq\{(4 \pi+c) / \pi\} \theta^{*}\left(u_{1}, u_{2}\right)$. Thus the result follows from Lemma 7

16. Asymptotic expressions for $Z(w)$ and $Z^{\prime}(w)$. We prove now the following

THEOREM X. If $S$ is an L-strip with boundary inclination 0 at $u=+\infty$, we have

(i) For $w=u+i v$ in $S$, uniformly in $v$,

$$
\lim _{u \rightarrow+\infty} \frac{Z(w)}{\int_{u_{0}}^{u} \frac{d t}{\theta(t)}}=\pi, \quad u_{0} \text { defined as in } \S 1(a) .
$$

(ii) Uniformly in any fixed strip $|y| \leqq \beta<\pi / 2(z=x+i y)\left({ }^{22}\right)$,

$$
\lim _{x \rightarrow+\infty} \frac{\left|W^{\prime}(z)\right|}{\theta(u)}=\frac{1}{\pi} \quad(u=U(z)), \quad \lim _{x \rightarrow+\infty} \frac{\log \left|W^{\prime}(z)\right|}{|z|}=0 .
$$

(iii) The straight line $\Lambda_{y}: y=$ const., $|y|<\pi / 2$, is mapped by $Z(w)$ onto a curve $L_{y}$ which for sufficiently large $u$ is represented by an equation of the form

$$
v=f_{y}(u) \equiv \psi(u)+\frac{\theta(u)}{\pi} y+o[\theta(u)] \quad \text { as } u \rightarrow+\infty,
$$

uniformly in $|y|<\pi / 2\left({ }^{23}\right)$.

(iv) Let the image of the strip

$$
\sum_{\alpha}: \quad\{-\infty<x<\infty,|y| \leqq \alpha\}, \quad 0<\alpha<\pi / 2,
$$

by means of $w=W(z)$ be $T_{\alpha}$, and let $S_{\beta}$ be the region

$$
\left\{u>u_{0},\left|\frac{v-\psi(u)}{\theta(u)}\right|<\frac{\beta}{\pi}\right\}, \quad 0<\beta<\frac{\pi}{2} .
$$

If $0<\alpha \pm \epsilon<\pi / 2, \epsilon>0$, then there exists an $N=N(\epsilon ; \alpha)$ such that the part of $T_{\alpha}$ which lies in $u \geqq N$, contains that part of $S_{\alpha-\epsilon}$ which is in $u \geqq N$ and is contained in $S_{\alpha+\epsilon}$.

(22) Since $\arg W^{\prime}(z) \rightarrow 0$ as $x \rightarrow+\infty$, the first of these relations implies that even

$$
\lim _{x \rightarrow+\infty} \frac{W^{\prime}(z)}{\theta(u)}=\frac{1}{\pi} .
$$

(23) This implies the following fact: If $\Lambda$ is a curve in the strip $|y|<\pi / 2$ which approaches the line $\Lambda_{t}$ as an asymptote, as $x \rightarrow+\infty$, then, for any point $(u, v)$ on the image $L$ of $\Lambda$ in the $w$-plane, $v=\psi(u)+(\theta(u) / \pi) t+o[\theta(u)]$ as $u \rightarrow+\infty$. ( $L$ need not be representable in the form $v=f(u)$.) For, if $\epsilon>0$ is given, there exists an $x_{1}$ such that the part of $\Lambda$ for $x \geqq x_{1}$ lies "between" the lines $\Lambda_{t-\epsilon}$ and $\Lambda_{t+\epsilon}$ (if $|t \pm \epsilon|<\pi / 2$; in case $t=\pi / 2$ or $t=-\pi / 2$, it lies "between" $\Lambda_{(\pi / 2)-\epsilon}$ and $\Lambda_{\pi / 2}$ or "between" $\Lambda_{-(\pi / 2)}$ and $\Lambda_{-(\pi / 2)+\epsilon}$, respectively). The image of that part of $\Lambda$ lies in a certain half-plane $u \geqq u_{1}$ "between" the curves $L_{t-\epsilon}$ and $L_{t+\epsilon}$ (or $L_{(\pi / 2)-\epsilon}, L_{\pi / 2}$ or $L_{-\pi / 2}, L_{-(\pi / 2)+\epsilon}$, respectively). Since $\epsilon$ may be taken arbitrarily small, this proves the above assertion. 
(v) In any region $S_{\beta}, 0<\beta<\pi / 2, \beta$ fixed,

$$
\lim _{u \rightarrow+\infty}\left[\left|Z^{\prime}(w)\right| \theta(u)\right]=\pi, \quad w=u+i v, w \in S_{\beta} .
$$

REMARK. Part (iii) may be considered as an extension in a certain direction of Carathéodory's well known result, which states that the map of the interior of a closed Jordan curve $\Gamma$ onto the circle $|\zeta|<1$ is "quasi-conformal" at a boundary point $\omega_{0}$ with a corner of measure $\beta>0$, i.e., that the angles at corresponding boundary points $\omega_{0}$ and $\zeta_{0}$ are transformed proportionally $\left({ }^{24}\right)$. Transformation of the interior of $\Gamma$ onto a strip $S$ by means of the function $w=\log \left[1 /\left(\omega-\omega_{0}\right)\right]$ and of $|\zeta|<1$ onto the strip $|y|<\pi / 2$, in such a way that $\zeta=\zeta_{0}$ corresponds to $x=+\infty$, leads to the following statement of Carathéodory's theorem: Let $C$ be a closed Jordan curve through $w=\infty$ which, in a neighborhood of $w=\infty$, consists of two branches $C_{+}$and $C_{-}$having the lines $v=\phi_{+}$and $v=\phi_{-}$, respectively, for asymptotes as $u \rightarrow+\infty\left(\phi_{+}-\phi_{-}=\theta>0\right)$. If $W(z)=U(z)+i V(z)$ maps $|y|<\pi / 2$ onto the interior $S$ of $C$ and if $\lim _{x \rightarrow+\infty} U(z)=+\infty$, then

$$
V(z)=\frac{1}{2}\left[\phi_{+}+\phi_{-}\right]+\frac{\theta}{\pi} y+o(1)
$$

as $x \rightarrow+\infty$,

uniformly in $|y|<\pi / 2$.

If $S$ is a simple Jordan strip, the hypothesis, regarding the asymptotes of $C_{+}$and $C_{-}$means that

$$
\lim _{u \rightarrow+\infty} \phi_{+}(u)=\phi_{+} \text {and } \lim _{u \rightarrow+\infty} \phi_{-}(u)=\phi_{-} \text {exist. }
$$

Similarly, Part (ii) can be considered as an extension in a certain direction of a theorem of Ostrowski which (formulated for infinite strips) states: Under the hypothesis of Carathéodory's theorem

$$
\lim _{x \rightarrow+\infty}\left|W^{\prime}(z)\right|=\frac{\theta}{\pi}, \quad \lim _{x \rightarrow+\infty}\left[\frac{\log \left|W^{\prime}(z)\right|}{|z|}\right]=0,
$$

uniformly in any strip $|y| \leqq \beta<\pi / 2$. Here $\theta$ may be greater than or equal to $0\left({ }^{25}\right)$.

(24) Carathéodory [1, pp. 40-41] and [2, pp. 19-93]. See also Lindelöf [1, p. 87]. Carathéodory's theorem has been generalized in two other directions: 1 . The assumption that $C_{+}$and $C_{-}$ approach distinct asymptotes has been replaced by the condition that $C_{+}$and $C_{-}$"oscillate" within the strips $\phi_{+}-k_{+} \leqq v \leqq \phi_{+}+k_{+}$and $\phi_{-}-k_{-} \leqq v \leqq \phi_{-}+k_{-}$respectively ( $k_{+}, k_{-}$constants). 2. It has been extended to the case where $C_{+}$and $C_{-}$may be arbitrary continua forming the boundary of $S$ (and not necessarily Jordan curves). For these extensions see Gross, [1, p. 278], Ostrowski [1, pp. 172-174], Wolff [2, p. 42] and [3, p. 46], Warschawski [2, p. 674]. Ostrowski $[2$, p. 77] gives a necessary and sufficient condition in order that the mapping function of a simply-connected region onto a half-plane preserve angles at an accessible boundary point.

$\left.{ }^{25}\right)$ Ostrowski [1]; the first of these equations is his relation (11.3) of page 101, the second follows, for $\theta>0$, by combination of his relations (62.2) and (62.1) of page 174, and, for $\theta=0$, by combination of his relations (68.7) of page 185 and (62.1) of page 174 . 
Both of these theorems do not require that $C_{+}$and $C_{-}$have an $L$-tangent at $u=+\infty$, as our Theorem $\mathbf{X}$ does. Our extension, however, refers to the fact that (iii) might be substituted for (16.3) when $C_{+}$and $C_{-}$have no asymptotes and that in (ii) the asymptotic behavior of $\left|W^{\prime}(z)\right|$ at $x=+\infty$ is given, whether $\theta(u)=\phi_{+}(u)-\phi_{-}(u)$ approaches a limit as $u \rightarrow+\infty$ or not.

17. Proof of Theorem X. (i) By hypothesis, $\psi^{\prime}(u)$ and $\theta^{\prime}(u)$ approach 0 as $u \rightarrow+\infty$. Hence it follows from Theorem IV (b) that there exists, for every $\epsilon>0$, an $N_{1}(\epsilon) \geqq u_{0}$ such that for all $w=u+i v$ and $w_{1}=u_{1}+i v_{1}$ in $S$ for which $u \geqq u_{1} \geqq N_{1}(\epsilon)$ :

$$
X(w)-X\left(w_{1}\right) \leqq \pi(1+\epsilon) \int_{u_{1}}^{u} \frac{d t}{\theta(t)}+\epsilon \leqq \pi(1+\epsilon) \int_{u_{0}}^{u} \frac{d t}{\theta(t)}+\epsilon .
$$

Hence,

$$
\frac{X(w)-X\left(w_{1}\right)}{\int_{u_{0}}^{u} \frac{d t}{\theta(t)}} \leqq \pi(1+\epsilon)+\frac{\epsilon}{\int_{u_{0}}^{u} \frac{d t}{\theta(t)}} .
$$

Keeping here $w_{1}$ fixed and letting $u \rightarrow+\infty$ we find, since $\int_{u_{0}}^{u}[\theta(t)]^{-1} d t \rightarrow \infty$ as $u \rightarrow+\infty$,

$$
\limsup _{u \rightarrow+\infty} \frac{X(w)}{\int_{u_{0}}^{u} \frac{d t}{\theta(t)}} \leqq \pi(1+\epsilon), \quad \text { uniforml } y \text { with respect to } v .
$$

Since the left-hand side of this inequality is independent of $\epsilon$, we may let $\epsilon \rightarrow 0$ and find

$$
\limsup _{u \rightarrow+\infty} \frac{X(w)}{\int_{u_{0}}^{u} \frac{d t}{\theta(t)}} \leqq \pi
$$

On the other hand, by Theorem III (a), uniformly with respect to $v$,

$$
\liminf _{u \rightarrow+\infty} \frac{X(w)}{\int_{u_{0}}^{u} \frac{d t}{\theta(t)}} \geqq \pi
$$

and (17.1) and (17.2) together imply that, uniformly in $v$,

$$
\lim _{u \rightarrow \infty} \frac{X(w)}{\int_{u_{0}}^{u} \frac{d t}{\theta(t)}}=\pi
$$


The result (i) follows now from (17.3), if we observe that $Z(w)=X(w)+i Y(w)$ and that $|Y(w)| \leqq \pi / 2$, while $\int_{u_{0}}^{u}[\theta(t)]^{-1} d t \rightarrow \infty$ as $u \rightarrow+\infty$.

(ii) Let $L$ denote the image in the $w$-plane of the real axis of the $z$-plane by means of $w=W(z)$. Let $z_{n}=3 n(n=1,2,3, \cdots)$ and $w_{n}=W\left(z_{n}\right)=u_{n}+i v_{n}$. Observing that $\lim _{u \rightarrow+\infty} \psi^{\prime}(u)=\lim _{u \rightarrow+\infty} \theta^{\prime}(u)=0$, we have, by Theorem IV (b), for all sufficiently large $n$,

or

$$
3=3(n+1)-3 n \leqq 2 \pi \int_{u_{n}}^{u_{n+1}} \frac{d u}{\theta(u)}+1,
$$

$$
\pi \int_{u_{n}}^{u_{n+1}} \frac{d u}{\theta(u)} \geqq 1
$$

Let $\epsilon>0$ be given. By Theorem IV (b) there exists an $n_{0}(\epsilon)$ such that for $n \geqq n_{0}(\epsilon)$

$$
\begin{aligned}
X\left(w_{n+1}\right)-X\left(w_{n}\right) & \leqq \pi\left(1+\frac{\epsilon}{2}\right) \int_{u_{n}}^{u_{n+1}} \frac{d u}{\theta(u)}+\frac{\epsilon}{2} \\
& \leqq \pi(1+\epsilon) \int_{u_{n}}^{u_{n+1}} \frac{d u}{\theta(u)},
\end{aligned}
$$

because of (17.4). Similarly, by Theorem III (b), there is an $n_{1}(\epsilon) \geqq n_{0}(\epsilon)$ such that for $n \geqq n_{1}(\epsilon)$ :

$$
X\left(w_{n+1}\right)-X\left(w_{n}\right) \geqq \pi \int_{u_{n}}^{u_{n+1}} \frac{d u}{\theta(u)}-\frac{\epsilon}{1+\epsilon} \geqq \frac{\pi}{1+\epsilon} \int_{u_{n}}^{u_{n+1}} \frac{d u}{\theta(u)} .
$$

Since arg $W^{\prime}(z) \rightarrow 0$ as $x \rightarrow+\infty$, the curve $L$ can be represented for suffciently large $u$ in the form $v=f(u)$ where $f^{\prime}(u)$ exists and approaches 0 as $u \rightarrow+\infty$. Evidently, for all sufficiently large $n, w_{n}=u_{n}+i f\left(u_{n}\right)$. The functions $X(u+i f(u))$ and $\int_{u_{n}}^{u}[\theta(t)]^{-1} d t$ are differentiable for $u_{n} \leqq u \leqq u_{n+1}$, and $d / d u \int_{u_{n}}^{u}[\theta(t)]^{-1} d t>0$ for $u \geqq u_{n}$. Hence, by the extended mean value theorem, for sufficiently large $n$, say $n \geqq n_{2} \geqq n_{1}$ :

$$
\frac{X\left(w_{n+1}\right)-X\left(w_{n}\right)}{\int_{u_{n}}^{u_{n}+1} \frac{d u}{\theta(u)}}=\theta\left(u_{n}^{\prime}\right)\left[\left.\frac{\partial X}{\partial u}\right|_{w_{n}^{\prime}}+\left.\frac{\partial X}{\partial v}\right|_{v_{n}^{\prime}} f^{\prime}\left(u_{n}^{\prime}\right)\right],
$$

where $u_{n}<u_{n}^{\prime}<u_{n+1}$ and $w_{n}^{\prime}=u_{n}^{\prime}+i f\left(u_{n}^{\prime}\right)$. Thus, we obtain from (17.5) and (17.6):

$$
\frac{\pi}{1+\epsilon} \leqq \theta\left(u_{n}^{\prime}\right)\left[\left.\frac{\partial X}{\partial u}\right|_{w_{n}^{\prime}}+\left.\frac{\partial X}{\partial v}\right|_{w_{n}^{\prime}} f^{\prime}\left(u_{n}^{\prime}\right)\right] \leqq \pi(1+\epsilon) .
$$


Since

$$
\frac{\partial X}{\partial u}=\left|Z^{\prime}(w)\right| \cos \left(\arg Z^{\prime}(w)\right), \quad \frac{\partial X}{\partial v}=-\left|Z^{\prime}(w)\right| \sin \left(\arg Z^{\prime}(w)\right)
$$

and

$$
\lim _{u \rightarrow+\infty}\left[\arg Z^{\prime}(w)\right]=\lim _{u \rightarrow+\infty} f^{\prime}(u)=0,
$$

we have, for all sufficiently large $n$, say $n \geqq n_{3} \geqq n_{2}$ :

$$
\frac{\pi}{(1+\epsilon)^{2}} \leqq \theta\left(u_{n}^{\prime}\right)\left|Z^{\prime}\left(w_{n}^{\prime}\right)\right| \leqq \pi(1+\epsilon)^{2} .
$$

Let $z_{n}^{\prime}=Z\left(w_{n}^{\prime}\right)$. Evidently $3 n \leqq z_{n}^{\prime} \leqq 3(n+1)$. Since $Z^{\prime}\left(w_{n}^{\prime}\right)=1 / W^{\prime}\left(z_{n}^{\prime}\right)$ we have also

$$
\frac{1}{\pi(1+\epsilon)^{2}} \leqq \frac{\left|W^{\prime}\left(z_{n}^{\prime}\right)\right|}{\theta\left(u_{n}^{\prime}\right)} \leqq \frac{(1+\epsilon)^{2}}{\pi} .
$$

Now let $z=x+i y$ be a point in the strip $|y| \leqq \beta$ with $x \geqq 3 n_{3}$, and let $n$ be such that $3 n \leqq x<3(n+1)$. Since then $\left|z_{n}^{\prime}-x\right| \leqq 3$, we infer from Theorem II (b) that, uniformly for $|y| \leqq \beta$,

$$
\lim _{x \rightarrow \infty}\left|\frac{W^{\prime}(z)}{W^{\prime}\left(z_{n}^{\prime}\right)}\right|=1 \text {. }
$$

Moreover, by the corollary of Lemma 7, we have for $u=U(z), 3 n \leqq x<3(n+1)$,

$$
\lim _{u \rightarrow+\infty} \frac{\theta(u)}{\theta\left(u_{n}^{\prime}\right)}=1 \text {. }
$$

Hence, if $x$ is sufficiently large,

$$
\frac{1}{\pi(1+\epsilon)^{3}} \leqq \frac{\left|W^{\prime}(z)\right|}{\theta(u)}=\left|\frac{W^{\prime}(z)}{W^{\prime}\left(z_{n}^{\prime}\right)}\right| \frac{\left|W^{\prime}\left(z_{n}^{\prime}\right)\right|}{\theta\left(u_{n}^{\prime}\right)} \frac{\theta\left(u_{n}^{\prime}\right)}{\theta(u)} \leqq \frac{(1+\epsilon)^{3}}{\pi},
$$

and this proves the first relation of part (ii) of the theorem.

To prove the second relation we use the first one:

$$
\log \left|W^{\prime}(z)\right|=\log \theta(u)-\log \pi+\log (1+\delta), \quad \lim _{x \rightarrow+\infty} \delta=0,
$$

uniformly in $|y| \leqq \beta$. Now, by part (i), uniformly in $|y|<\pi / 2$, as $x \rightarrow+\infty$,

$$
\frac{\log \theta(u)}{|z|} \sim \frac{\int_{u_{1}}^{u} \frac{\theta^{\prime}(t)}{\theta(t)} d t+\log \theta\left(u_{1}\right)}{\pi \int_{u_{1}}^{u} \frac{d t}{\theta(t)}} .
$$


Here $u_{1}$ may be chosen arbitrarily large, but fixed. Let $\epsilon>0$ be given. We choose $u_{1}$ such that $\left|\theta^{\prime}(t)\right|<\epsilon$ for $t \geqq u_{1}$. Then by the mean value theorem

$$
\frac{\left|\int_{u_{1}}^{u} \frac{\theta^{\prime}(t)}{\theta(t)} d t\right|}{\int_{u_{1}}^{u} \frac{d t}{\theta(t)}}=\left|\theta^{\prime}(\xi)\right|<\epsilon, \quad u_{1}<\xi<u .
$$

Keeping $u_{1}$ fixed and letting $u \rightarrow+\infty$, and hence $x \rightarrow+\infty$, we find from (17.8)

$$
\limsup _{x \rightarrow+\infty}\left|\frac{\log \theta(u)}{z}\right| \leqq \epsilon,
$$

and the result follows from (17.7).

(iii) a. We shall first prove the weaker statement that (16.1) holds uniformly for all $y$ with $|y| \leqq \beta, \beta$ being any fixed positive number less than $\pi / 2$.

The image $L_{\nu}$ of $\Lambda_{\nu}$ is represented in parametric form by the equations $u=U(x+i y), v=V(x+i y)$, where $x$ is the parameter, $-\infty<x<\infty$, and $y$ is fixed. Evidently

$$
V(x+i y)=V(x)+\int_{0}^{\nu} \frac{\partial V(x+i \eta)}{\partial \eta} d \eta .
$$

For $\zeta=x+i \eta,|\eta| \leqq|y|$

$$
\frac{\partial V(x+i \eta)}{\partial \eta}=R\left\{\frac{d W(\zeta)}{d \zeta}\right\}=\left|W^{\prime}(\zeta)\right| \cos \left(\arg W^{\prime}(\zeta)\right),
$$

and by Theorem II (a), the first of the relations (ii), and the corollary of Lemma 7 , we have for $u=U(x)$

$$
\frac{\partial V(\zeta)}{\partial \eta}=\frac{\theta(u)}{\pi}(1+o(1))
$$
as $u \rightarrow+\infty$,

uniformly for $|\eta| \leqq \beta$. Therefore,

$$
V(x+i y)=V(x)+\frac{\theta(u)}{\pi} y+o[\theta(u)], \quad \text { as } x \rightarrow+\infty,
$$

uniformly for $|y| \leqq \beta$.

Since $\arg W^{\prime}(z) \rightarrow 0$ as $x \rightarrow+\infty$, uniformly in $|y|<\pi / 2$, there exists an $N^{\prime}$ such that for $u \geqq N^{\prime}$ every curve $L_{y},|y|<\pi / 2$, is representable in the form $v=f_{y}(u)$, where $d f_{y}(u) / d u$ is continuous and approaches 0 as $u \rightarrow+\infty$, uniformly for $|y|<\pi / 2$. Let $u=U(x), u^{\prime}=U(x+i y)$ and let $x$ be so large that $u$, $u^{\prime} \geqq N^{\prime \prime} \geqq N^{\prime}$, where $N^{\prime \prime}$ is so chosen that $\left|d f_{y}(u) / d u\right|<1$ for $u \geqq N^{\prime \prime}$. Hence $\left({ }^{26}\right)$

${ }^{(26)} \theta^{*}\left(u, u^{\prime}\right)=\max \theta(t)$ for $t$ between $u$ and $u^{\prime}$. 


$$
\left|f_{y}(u)-V(x+i y)\right|=\left|f_{y}(u)-f_{y}\left(u^{\prime}\right)\right|<\left|u-u^{\prime}\right| \leqq \theta^{*}\left(u, u^{\prime}\right)\left|\int_{u}^{u^{\prime}} \frac{d t}{\theta(t)}\right| .
$$

By Theorem III (b), $\int_{u}^{u^{\prime}}[\theta(t)]^{-1} d t=o(1)$ and, by the Corollary of Lemma 7, $\theta^{*}\left(u, u^{\prime}\right) / \theta(u) \rightarrow 1$ as $u \rightarrow+\infty$. We find therefore

$$
\left|f_{y}(u)-V(x+i y)\right| \leqq \theta(u) o(1),
$$

as $u \rightarrow+\infty$,

uniformly in $|y|<\pi / 2$. Hence we obtain from (17.10)

$$
f_{v}(u)=V(x)+\frac{\theta(u)}{\pi} y+o[\theta(u)] \quad \text { as } u \rightarrow+\infty,
$$

uniformly for $|y| \leqq \beta$.

Let now an $\epsilon, 0<\epsilon<\pi / 8$, be assigned. We may apply (17.11) for the particular value of $\beta=\pi / 2-\epsilon$. Let the curves $L_{y}$ for $y=\pi / 2-\epsilon$ and $y=-\pi / 2+\epsilon$, for sufficiently large $u$, be represented by the equations $v=f_{+}(u)$ and $v=f_{-}(u)$, respectively. Then it follows from (17.11) that

$$
\begin{aligned}
& f_{+}(u)=V(x)+\theta(u)\left[\frac{1}{2}-\frac{\epsilon}{\pi}\right]+o[\theta(u)], \\
& f_{-}(u)=V(x)+\theta(u)\left[\frac{\epsilon}{\pi}-\frac{1}{2}\right]+o[\theta(u)]
\end{aligned}
$$

as $u \rightarrow+\infty$. Addition and subtraction of these expressions give

$$
\begin{aligned}
V(x) & =\frac{f_{+}(u)+f_{-}(u)}{2}+o[\theta(u)], \\
f_{+}(u)-f_{-}(u) & =\theta(u)\left[1-\frac{2 \epsilon}{\pi}\right]+o[\theta(u)],
\end{aligned}
$$

respectively. Substituting $\theta(u)=\phi_{+}(u)-\phi_{-}(u)$ into the second expression we find

$$
\left[\phi_{+}(u)-f_{+}(u)\right]+\left[f_{-}(u)-\phi_{-}(u)\right]=\frac{2 \epsilon}{\pi} \theta(u)+o[\theta(u)]
$$

Since each of the summands on the left-hand side is greater than or equal to 0 ,

$$
\begin{aligned}
& 0 \leqq \phi_{+}(u)-f_{+}(u) \leqq\left[\frac{2 \epsilon}{\pi}+o(1)\right] \theta(u), \\
& 0 \leqq f_{-}(u)-\phi_{-}(u) \leqq\left[\frac{2 \epsilon}{\pi}+o(1)\right] \theta(u) .
\end{aligned}
$$

Hence we obtain from the first of the relations (17.12) 


$$
V(x)=\frac{\phi_{+}(u)+\phi_{-}(u)}{2}+\left[\lambda \frac{2 \epsilon}{\pi}+o(1)\right] \theta(u)
$$

for a suitable $\lambda,|\lambda| \leqq 1$. Substitution of (17.13) into (17.11) gives

$$
f_{y}(u)=\frac{1}{2}\left(\phi_{+}(u)+\phi_{-}(u)\right)+\frac{\theta(u)}{\pi} \cdot y+\left[\frac{2 \epsilon}{\pi} \lambda+o(1)\right] \theta(u) \quad \text { as } u \rightarrow+\infty,
$$

uniformly for $|y| \leqq \beta$. This proves our result in the above stated weaker form, since it shows that, for all $|y| \leqq \beta$,

$$
\left|f_{y}(u)-\psi(u)-\frac{\theta(u)}{\pi} y\right| \leqq\left[\frac{2 \epsilon}{\pi}+o(1)\right] \theta(u)<\epsilon \theta(u),
$$

for all sufficiently large $u$, say $u \geqq N=N(\epsilon ; \beta) \geqq N^{\prime \prime}$.

b. Using this (weaker) result only we shall prove part (iv) of our theorem below. Anticipating here part (iv), we can readily prove (16.1) in the complete form, namely that $o[\theta(u)]$ in (16.1) holds uniformly for $|y|<\pi / 2$.

Let $\epsilon, 0<\epsilon<\pi / 8$, be given. Choose $\beta=\pi / 2-\epsilon / 2$ and determine the index $N(\epsilon ; \pi / 2-\epsilon / 2)$ so that (17.14) holds. Let $\pi / 2-\epsilon / 2<y<\pi / 2$. Then, by part (iv), there exists an $N_{1}(\epsilon) \geqq N$ such that all points $w=u+i f_{y}(u)$ with $u \geqq N_{1}$ lie in the exterior of $S_{\beta-\epsilon / 2}$, i.e., either

or

$$
\psi(u)+\frac{\theta(u)}{\pi}\left(\frac{\pi}{2}-\epsilon\right)<f_{y}(u)<\phi_{+}(u)
$$

$$
\phi_{-}(u)<f_{y}(u)<\psi(u)+\frac{\theta(u)}{\pi}\left(\epsilon-\frac{\pi}{2}\right) .
$$

Since we assumed $\pi / 2-\epsilon / 2<y<\pi / 2$ and $\lim _{y \rightarrow \pi / 2} f_{y}(u)=\phi_{+}(u)$, the first inequality holds. Similarly, if $-\pi / 2<y<-\pi / 2+\epsilon / 2$, the second inequality holds. Hence, for all $u \geqq N_{1}(\epsilon)$,

$$
\left|f_{y}(u)-\psi(u)-\frac{\theta(u)}{\pi} y\right|<\epsilon \theta(u) \quad \text { for all }|y|<\pi / 2 .
$$

(iv) Let $\epsilon$ be given as stated in the theorem. By (17.14) there exists an $N=N(\epsilon / \pi ; \alpha)$ such that all points $w \in T_{\alpha}$ with $R w \geqq N$ are cortained in the region

$$
\psi(u)-\frac{\theta(u)}{\pi} \alpha-\frac{\theta(u)}{\pi} \epsilon<v<\psi(u)+\frac{\theta(u)}{\pi} \alpha+\frac{\theta(u)}{\pi} \epsilon, \quad u \geqq N .
$$

This proves that the part of $T_{\alpha}$ which lies in $u \geqq N$ is contained in $S_{\alpha+\epsilon}$. In a similar manner it may be seen that it contains the part of $S_{\alpha-\epsilon}$ within $u \geqq N$.

(v) Relation (16.2) follows immediately from the first of the relations (ii) and part (iv) if we observe that, for $w=W(z), Z^{\prime}(w)=1 / W^{\prime}(z)$. 
18. Corollaries of Theorem X. We now prove the following corollaries.

COROLlary 1. If $S$ is an L-strip with boundary inclination $\gamma,|\gamma|<\pi / 2$ at $u=+\infty$, then parts (iii) and (iv) of Theorem $\mathrm{X}$ remain unchanged and parts (ii) and $(\mathrm{v})$ are to be replaced by the relations

(ii $\left.{ }^{*}\right) \lim _{x \rightarrow+\infty}\left|W^{\prime}(z)\right| / \theta(u)=(\cos \gamma) / \pi, \lim _{x \rightarrow+\infty}\left[\log \left|W^{\prime}(z)\right|\right] /|z|=0$, uniformly in $y$,

$\left(\mathrm{v}^{*}\right) \lim _{u \rightarrow+\infty}\left[\left|Z^{\prime}(w)\right| \theta(u)\right]=\pi / \cos \gamma$, uniformly for $w=u+i v \in S_{\beta}$, $0<\beta<\pi / 2$, respectively. Moreover, uniformly for $w \in S$,

$$
Y(w)=\pi \frac{v-\psi(u)}{\theta(u)}+o(1) \quad \text { as } u \rightarrow+\infty .
$$

To prove the corollary we rotate the coordinate system through the angle $\gamma$ in the positive direction, thus obtaining a new set of coordinates $(\bar{u}, \bar{v})$, $\bar{u}+i \bar{v}=\bar{w}$, where $\bar{w}=e^{-i \gamma} w$. Let, for sufficiently large $\bar{u}, \bar{v}=\bar{\phi}_{+}(\bar{u})$ and $\bar{v}=\bar{\phi}_{-}(\bar{u})$ represent $C_{+}$and $C_{-}$, respectively. Consider a point $C: w=u+i v \in S$ and let $w=e^{-i \gamma} w$. Denote the points $u+i \phi_{-}(u), u+i \phi_{+}(u)$, in the $w$-plane, and $\bar{u}+i \bar{\phi}_{-}(\bar{u}), \bar{u}+i \bar{\phi}_{+}(\bar{u})$ in the $\bar{v}$-plane by $A, B, \bar{A}, \bar{B}$ respectively. In the triangle $\bar{A} A C$, the angles $\bar{A}$ and $A$ approach $\pi / 2$ and $\pi / 2-|\gamma|$ respectively, and in $\bar{B} B C$, the angles $\bar{B}$ and $B$ approach $\pi / 2$ and $\pi / 2-|\gamma|$ respectively as $u \rightarrow+\infty$, uniformly with respect to the position of $C$ on $A B$. Hence

$$
\frac{A C}{\bar{A} C}=\frac{\sin \bar{A}}{\sin A} \rightarrow \frac{1}{\cos \gamma}, \quad \frac{B C}{\bar{B} C} \rightarrow \frac{1}{\cos \gamma} \quad \text { as } u \rightarrow+\infty .
$$

and therefore $\left(\bar{\theta}(\bar{u})=\bar{\phi}_{+}(\bar{u})-\bar{\phi}_{-}(\bar{u})\right)$

$$
\theta(u)=A B=A C+B C=\frac{\bar{A} \bar{B}}{\cos \gamma}+o(\bar{A} \bar{B})=\frac{\bar{\theta}(\bar{u})}{\cos \gamma}(1+o(1))
$$

as $u \rightarrow+\infty$,

the convergence in (18.2) and (18.3) being uniform with respect to the position of $C$ on $A B$.

Now (ii*) follows immediately if we observe that for $\bar{W}(z)=e^{-i \gamma} W(z)$ by Theorem X (ii) and subsequently by (18.3), uniformly for $|y| \leqq \beta$ :

$$
\frac{1}{\pi}=\lim _{x \rightarrow+\infty} \frac{\left|\bar{W}^{\prime}(z)\right|}{\bar{\theta}(\bar{u})}=\lim _{x \rightarrow+\infty} \frac{\left|W^{\prime}(z)\right|}{\theta(u) \cos \gamma}, \quad \bar{u}=R \bar{W}(z) ; u=R W(z),
$$

and that

$$
\frac{\log \left|\bar{W}^{\prime}(z)\right|}{|z|}=\frac{\log \left|W^{\prime}(z)\right|}{|z|} .
$$

To prove now that part (iii) of Theorem $\mathrm{X}$ remains unchanged, we note that in the figure which was used above, $A C=v-\phi_{-}(u), B C=\phi_{+}(u)-v$, 
$\bar{A} C=\bar{v}-\bar{\phi}_{-}(\bar{u}), \bar{B} C=\bar{\phi}_{+}(\bar{u})-\bar{v}$. From (18.2) we obtain therefore (leaving off the arguments $u, \bar{u})$

$$
v-\phi_{-}=\left(\bar{v}-\bar{\phi}_{-}\right) \frac{1}{\cos \gamma}+o(\bar{\theta}), \quad \phi_{+}-v=\left(\bar{\phi}_{+}-\bar{v}\right) \frac{1}{\cos \gamma}+o(\bar{\theta}),
$$

as $u \rightarrow+\infty$, uniformly with respect to the position of $C$ on $A B$. By Theorem $\mathrm{X}$ (iii), there exists an $\bar{N}$ (independent of $y$ ), such that any line $\Lambda_{y}: y=$ const. $(|y|<\pi / 2)$ is mapped by $\bar{w}=\bar{W}(z)$ onto a curve $L_{y}$ which, for all $\bar{u} \geqq \bar{N}$, can be represented in the form $\left(\bar{\psi}=\frac{1}{2}\left[\bar{\phi}_{+}+\bar{\phi}_{-}\right]\right)$:

$$
\bar{v}=\bar{\psi}+\frac{\bar{\theta}}{\pi} y+o(\bar{\theta}) \quad \text { as } \bar{u} \rightarrow+\infty, \text { uniforml } y \text { for }|y|<\pi / 2 .
$$

Hence, for $\bar{w}=\bar{u}+\bar{v} i$ on $L_{y}(\bar{u} \geqq \bar{N})$, uniformly for $|y|<\pi / 2$,

$$
\bar{v}-\bar{\phi}_{-}=\frac{\bar{\theta}}{\pi}\left[\frac{\pi}{2}+y+o(1)\right], \quad \bar{\phi}_{+}-\bar{v}=\frac{\bar{\theta}}{\pi}\left[\frac{\pi}{2}-y+o(1)\right]
$$

as $u \rightarrow+\infty$. Substituting these values for $\bar{v}-\bar{\phi}_{-}$and $\bar{\phi}_{+}-\bar{v}$ into the first and second equations of (18.4), respectively and using (18.3) we find that there exists an $N$ (independent of $y$ ) such that any point $w=u+i v$ on $L_{y}$ with $u \geqq N$ satisfies the relations

$$
v-\phi_{-}=\frac{\theta}{\pi}\left[\frac{\pi}{2}+y+o(1)\right], \quad \phi_{+}-v=\frac{\theta}{\pi}\left[\frac{\pi}{2}-y+o(1)\right],
$$

as $u \rightarrow+\infty$, uniformly in $|y|<\pi / 2$. Subtraction of the second equation in (18.5) from the first gives

$$
v=\psi+\frac{\theta}{\pi} y+o(\theta) \quad \text { as } u \rightarrow+\infty, \text { uniformly in }|y|<\pi / 2 .
$$

It may be shown now by use of (18.6) (as in the proof of part (iv) of Theorem X) that the statement of part (iv) remains unchanged in the case of any $\gamma$, $|\gamma|<\pi / 2$. Part $\left(v^{*}\right)$ of the corollary follows then from part (ii*) in the same manner as part (v) of the theorem follows from part (ii).

Finally, (18.1) is obtained by solving (18.6) for $y$, since for $w=u+i v$ on $L_{t}$ : $Y(w)=t$.

COROLlaRy 2. Let $S$ be an L-strip with the boundary inclination $\gamma$, $|\gamma|<\pi / 2$, at $u=+\infty$.

(i) If $\gamma=0$, then for $z_{1}=x_{1}+i y_{1}, z_{2}=x_{2}+i y_{2},\left|x_{2}-x_{1}\right| \leqq M$ (M=const.),

$$
U\left(z_{2}\right)-U\left(z_{1}\right)=\frac{\theta(u)}{\pi}\left(x_{2}-x_{1}\right)+o[\theta(u)]
$$


uniformly for $|y|<\pi / 2$ as $x_{1}, x_{2} \rightarrow+\infty$. Here u may be taken equal to any number between $u_{1}$ and $u_{2}$.

In particular, if $\lim _{u \rightarrow+\infty} \theta(u)=\theta$ exists, then (18.7) becomes

$$
U\left(z_{2}\right)-U\left(z_{1}\right)=\frac{\theta}{\pi}\left(x_{2}-x_{1}\right)+o(1) .
$$

(ii) If $\lim \sup _{u \rightarrow+\downarrow} \theta(u)=\theta^{*}, \lim _{\inf _{u \rightarrow+\infty}} \theta(u)=\theta_{*}$, then, uniformly in $|y|<\pi / 2$,

$$
\frac{\theta_{*} \cos ^{2} \gamma}{\pi} \leqq \liminf _{x \rightarrow+\infty} \frac{U(z)}{x} \leqq \limsup _{x \rightarrow+\infty} \frac{U(z)}{x} \leqq \frac{\theta^{*} \cos ^{2} \gamma}{\pi} .
$$

Thus, if $\lim _{u \rightarrow+\infty} \theta(u)=\theta$ exists:

$$
\lim _{x \rightarrow+\infty} \frac{U(z)}{x}=\frac{\theta \cos ^{2} \gamma}{\pi}, \quad \lim _{x \rightarrow+\infty} \frac{W(z)}{z}=\frac{\theta \cos \gamma}{\pi} e^{i \gamma} .
$$

REMARK. In the case that

$$
\lim _{u \rightarrow+\infty} \phi_{+}(u)=\phi_{+}, \quad \lim _{u \rightarrow+\infty} \phi_{-}(u)=\phi_{-} \text {exist, }
$$

both of these parts are known theorems $\left({ }^{27}\right)$.

Proof. (i) Let $u_{1}=U\left(z_{1}\right), u_{2}=U\left(z_{2}\right), x_{2} \geqq x_{1}$. By Theorems III (b) and IV (b)

$$
\begin{aligned}
-\epsilon_{1}+\pi \int_{u_{1}}^{u_{2}} \frac{d u}{\theta(u)} \leqq x_{2}-x_{1} \leqq \pi\left(1+\epsilon_{2}\right) & \int_{u_{1}}^{u_{2}} \frac{d u}{\theta(u)}+\epsilon_{3}, \\
& \lim _{x_{1} \rightarrow+\infty} \epsilon_{k}=0, k=1,2,3 .
\end{aligned}
$$

By the corollary of Lemma 7 , for any $u$ between $u_{1}$ and $u_{2}$ :

$$
\int_{u_{1}}^{u_{2}} \frac{d t}{\theta(t)}=\frac{1}{\theta(u)}\left(u_{2}-u_{1}\right)\left(1+\epsilon_{4}\right), \quad \lim _{x_{1} \rightarrow+\infty} \epsilon_{4}=0 .
$$

Substituting this value of $\int_{u_{1}}^{u_{2}}[\theta(t)]^{-1} d t$ into (18.10) and observing that $\left|x_{2}-x_{1}\right| \leqq M$, we find (18.7).

(27) In the case that $\phi_{+}-\phi_{-}=\theta>0,\left(18.7^{*}\right)$ has been proved under the less restrictive hypothesis that $S$ is a region as described in the statement of Caratheodory's theorem in the remark of $\$ 16$, whose boundary curves $C_{+}$and $C_{-}$(which approach the aysmptotes $v=\phi_{+}$and $v=\phi_{-}$respectively) satisfy an additional condition regarding their "oscillation" (Reguläre Unbewalltheit"). See Wolff [1, p. 217], Warschawski [1, p. 326], Ostrowski [1, p. 117, relation (21.2)]. For $\theta=0$, Ostrowski [1, p. 177] proved $\left(18.7^{*}\right)$ under the assumption that $S$ is an $L$-strip satisfying (18.9). Compare also Ostrowski's extension of the first case $(\theta>0)$ to regions with general boundaries [2, pp. 88, 95].-Part (ii) of Corollary 2, under the assumption (18.9) is due to Ostrowski $[1$, p. 174, relation (62.1)]. 
(ii) Assume first that $\gamma=0$. Then it is sufficient to prove that

$$
\frac{\theta_{*}}{\pi} \leqq \liminf _{u \rightarrow+\infty} \frac{u}{X(w)} \leqq \limsup _{u \rightarrow+\infty} \frac{u}{X(w)} \leqq \frac{\theta^{*}}{\pi}, \quad \text { uniformly for } w \in S .
$$

Let $\epsilon>0$ be given. Take $u_{1}$ so large that

$$
\theta_{*}-\epsilon \leqq \theta(u) \leqq \theta^{*}+\epsilon
$$

for $u \geqq u_{1}$.

By Theorem X (i), uniformly for $w \in S$, as $u \rightarrow+\infty$,

$$
\frac{u}{X(w)} \sim \frac{u}{\pi \int_{u_{1}}^{u} \frac{d t}{\theta(t)}}=\frac{u}{u-u_{1}} \cdot \frac{\theta(\xi)}{\pi}, \quad u_{1}<\xi<u .
$$

Hence, keeping $u_{1}$ fixed, we have

$$
\frac{\theta_{*}-\epsilon}{\pi} \leqq \liminf _{u \rightarrow+\infty} \frac{u}{X(w)} \leqq \lim \sup \frac{u}{X(w)} \leqq \frac{\theta^{*}+\epsilon}{\pi} .
$$

Since $\epsilon$ is arbitrary this proves (18.11).

If $\gamma \neq 0$, let $\imath \bar{v}=e^{-i \gamma} w, \bar{W}(z)=\bar{U}(z)+i \bar{V}(z)=e^{-i \gamma} W(z)$. Moreover, let $\bar{\theta}(\bar{u})$ be defined in the $\bar{w}$-plane as $\theta(u)$ is in the $w$-plane. Then, by the part just proved,

$$
\frac{1}{\pi} \liminf _{\bar{u} \rightarrow+\infty} \bar{\theta}(\bar{u}) \leqq \liminf _{x \rightarrow+\infty} \frac{\bar{U}(z)}{x} \leqq \limsup _{x \rightarrow+\infty} \frac{\bar{U}(z)}{x} \leqq \frac{1}{\pi} \limsup _{\bar{u} \rightarrow+\infty} \bar{\theta}(\bar{u}) .
$$

Now

$$
U(z)=\bar{U}(z) \cos \gamma-\bar{V}(z) \sin \gamma=\bar{U}(z) \cos \gamma\left\{1-\frac{\bar{V}(z)}{\bar{U}(z)} \tan \gamma\right\} .
$$

Here $\bar{V}(z) / \bar{U}(z) \rightarrow 0$ as $x \rightarrow+\infty$, uniformly for $|y|<\pi / 2$. Using this relation in connection with (18.3) we obtain (18.8) from (18.12).

\section{Applications}

19. The general result. We shall now apply our results to the study of the mapping function in a neighborhood of a finite boundary point $\omega_{0}$ of a region bounded by a closed Jordan curve. First we derive from our results a theorem which deals with a certain general boundary configuration and then apply it to various special cases.

(a) Let $R$ be the interior of a closed Jordan curve $\Gamma$ in the $\omega$-plane and let $\omega=0$ be on $\Gamma$. Suppose that in a neighborhood of $\omega=0$, say $|\omega| \leqq a, \Gamma$ consists of two arcs $\Gamma_{+}$and $\Gamma_{-}$which are represented in polar coordinates in the form

$$
\begin{aligned}
\phi=\Phi_{+}(\rho), \quad \phi= & \Phi_{-}(\rho), \\
& 0<\rho \leqq a, \quad \Phi_{+}(\rho)<\Phi_{-}(\rho),
\end{aligned}
$$


respectively, the functions $\Phi_{+}(\rho), \Phi_{-}(\rho)$ being continuous in the interval $0<\rho \leqq a$. The region

$$
0<\rho<a, \quad \Phi_{+}(\rho)<\phi<\Phi_{-}(\rho)
$$

is contained in $R$. We set $\Theta(\rho) \equiv \Phi_{-}(\rho)-\Phi_{+}(\rho)$ and $\Psi(\rho)=\frac{1}{2}\left[\Phi_{-}(\rho)+\Phi_{+}(\rho)\right]$.

Suppose that $\Phi_{+}(\rho)$ and $\Phi_{-}(\rho)$ are absolutely continuous in any closed interval within $0<\rho \leqq a$ and that $\rho\left[d \Phi_{+}(\rho) / d \rho\right]$ and $\rho\left[d \Phi_{-}(\rho) / d \rho\right]$, which exist for $0<\rho \leqq a$ except possibly for a set of measure 0, approach the same limit, $\tan \gamma$, $|\gamma|<\pi / 2$, as $\rho \rightarrow 0$.

Finally, let $\zeta=\zeta(\omega)$ map $R$ conformally onto the circle $|\zeta-1|<1$ in such a manner that $\omega=0$ corresponds to $\zeta=0$ and let $\omega=\omega(\zeta)$ denote its inverse function.

(b) Logarithmic transformation of $R$ by means of the function $\left({ }^{28}\right)$ $w=\log (1 / \omega)$ and of the circle $|\zeta-1|<1$ by means of $z=\log [(2-\zeta) / \zeta]$ gives at once the following results:

THEOREM XI(A). Under the above stated hypotheses we have $\left({ }^{29}\right)$ :

(i) If $\gamma=0$, then for any branch of $\log \zeta(\omega),|\omega|=\rho$, uniformly in $R$,

$$
\lim _{\rho \rightarrow 0} \frac{\log \zeta(\omega)}{\int_{\rho}^{a} \frac{d r}{r \Theta(r)}}=-\pi .
$$

(ii) Uniformly in any angle $|\arg \zeta| \leqq \beta<\pi / 2$, as $\zeta \rightarrow 0$,

$$
\frac{\left|\omega^{\prime}(\zeta)\right|}{\left|\frac{\omega(\zeta)}{\zeta}\right|} \sim \frac{\Theta(\rho)}{\pi} \cos \gamma, \quad|\omega|=\rho .
$$

(iii) Any circular arc $\lambda_{t}, t$ fixed, $|t|<\pi / 2:\{\arg \{(2-\zeta) / \zeta\}=t,|\zeta-1|<1\}$ is mapped by $\omega(\zeta)$ onto a curve $l_{t}$ which in a neighborhood of $\omega=0$ is representable in the form

$$
\phi=\Psi(\rho)-\frac{\Theta(\rho)}{\pi} t+o[\Theta(\rho)] \quad \text { as } \rho \rightarrow 0,
$$

uniformly for $|t|<\pi / 2$.

${ }^{(28)} R$ is transformed by $w=\log 1 / \omega$ into an $L$-strip $S$ whose boundary curves $C_{+}$and $C_{-}$ are given by the equations $v=\phi_{+}(u)=-\Phi_{+}\left(e^{-u}\right), v=\phi_{-}(u)=-\Phi_{-}\left(e^{-u}\right)$, respectively. To see that $C_{+}$(and similarly $C_{-}$) has an $L$-tangent at $u=+\infty$, one only has to note that

where $\epsilon \rightarrow 0$ as $\rho_{1}, \rho_{2} \rightarrow 0$.

$$
\begin{aligned}
\frac{\phi_{+}\left(u_{2}\right)-\phi_{+}\left(u_{1}\right)}{u_{2}-u_{1}} & =-\frac{1}{u_{2}-u_{1}} \int_{\rho_{1}}^{\rho_{2}} \frac{d \Phi_{+}}{d \rho} d \rho=-(1+\epsilon) \frac{\tan \gamma}{u_{2}-u_{1}} \int_{\rho_{1}}^{\rho_{2}} \frac{d \rho}{\rho} \\
& =-(1+\epsilon) \tan \gamma \frac{\log \rho_{2}-\log \rho_{1}}{u_{2}-u_{1}}=(1+\epsilon) \tan \gamma,
\end{aligned}
$$

${ }^{(29)}$ Part (A) follows from Theorem $\mathrm{X}$ and Corollary 1 of this theorem. 
(iv) Let $\sigma_{\alpha}$ denote the region within $|\zeta-1|<1$ which is bounded by the two circular arcs arg $\{(2-\zeta) / \zeta\}=\alpha$ and $\arg \{(2-\zeta) / \zeta\}=-\alpha, 0<\alpha<\pi / 2$, let $\tau_{\alpha}$ be its image in the $\omega$-plane by means of $\omega(\zeta)$, and let $s_{\beta}(0<\beta<\pi / 2)$ denote the region $\{0<\rho<a,|\phi-\Psi(\rho)| \leqq(\beta / \pi) \Theta(\rho)\}$.

If $0<\alpha \pm \epsilon<\pi / 2, \epsilon>0$, then there exists an $r=r(\epsilon ; \alpha)$ such that the part of $\tau_{\alpha}$ which lies in $0<\rho \leqq r$ contains that part of $s_{\alpha-\epsilon}$ which is in $\rho \leqq r$ and is contained in $s_{\alpha+e}$.

(v). Uniformly in any region $s_{\beta}, 0<\beta<\pi / 2$,

$$
\frac{\left|\zeta^{\prime}(\omega)\right|}{\left|\frac{\zeta(\omega)}{\omega}\right|} \sim \frac{\pi}{\Theta(\rho) \cos \gamma} \quad \text { as }|\omega|=\rho \rightarrow 0 .
$$

(vi) Uniformly in $R$, for $\omega=\rho e^{i \phi}$,

$$
\arg \zeta(\omega)=\pi \frac{\phi-\Psi(\rho)}{\Theta(\rho)}+o(1) \quad \text { as } \omega \rightarrow 0 .
$$

THEOREM XI (B). If in addition to the hypotheses stated in $\$ 19$ (a), $\rho\left[d \Phi_{+}(\rho) / d \rho\right]$ and $\rho\left[d \Phi_{-}(\rho) / d \rho\right]$ are continuous for $0 \leqq \rho \leqq a$ and the integrals

$$
\int_{\rho=0}^{a}\left|d\left(\rho \Phi_{+}^{\prime}(\rho)\right)\right|, \quad \int_{\rho=0}^{a}\left|d\left(\rho \Phi_{-}^{\prime}(\rho)\right)\right|, \quad \int_{0}^{a} \frac{\Theta^{\prime 2}(\rho)}{\Theta(\rho)} d \rho \text { converge, }
$$

then there exists a constant $c>0$ such that, for $\omega=\rho e^{i \phi}$,

$$
|\zeta(\omega)|=c \exp \left\{-\pi \int_{\rho}^{a} \frac{1+\left(r \Psi^{\prime}(r)\right)^{2}}{r \Theta(r)} d r+\pi \frac{\phi-\Psi(\rho)}{\Theta(\rho)} \tan \gamma+o(1)\right\}
$$

as $\omega \rightarrow 0$ in any way at all in $R\left({ }^{30}\right)$.

(c) REMARK. If $\Gamma$ is a curve as described in $\$ 19$ (a) and if, in addition the integrals

$$
\int_{0}^{a} \rho\left(\frac{d \Phi_{+}}{d \rho}\right)^{2} \frac{d \rho}{\Theta(\rho)} \text { and } \int_{0}^{a} \rho\left(\frac{d \Phi_{-}}{d \rho}\right)^{2} \frac{d \rho}{\Theta(\rho)} \text { converge, }
$$

then $\gamma=0\left({ }^{31}\right)$ and the integral $\int_{0}^{a}\left(\rho \Psi^{\prime 2}(\rho) / \Theta(\rho)\right) d \rho$ exists, and therefore by the corollary of Theorem IV (\$7), (19.3) reduces to

$$
|\zeta(\omega)|=c^{\prime} \exp \left\{-\pi \int_{\rho}^{a} \frac{d r}{r \Theta(r)}+o(1)\right\} \text { as } \omega \rightarrow 0 \text { in } R\left(c^{\prime}=\text { const. }\right) .
$$

(d) CoROllary. Let $R$ be a region in the w-plane satisfying the hypotheses

${ }^{\left({ }^{30}\right)}$ Part (B) follows from the corollary to Theorem VIII ( $\left.\$ 14\right)$.

(31) If $\gamma$ were not 0 , the convergence of either of the integrals (19.4) would imply that of $\int_{0}^{a}[r \Theta(r)]^{-1} d r$, and this integral diverges. 
of $\$ 19$ (a). If in addition, $\lim _{\rho \rightarrow 0} \Theta(\rho)=\theta$ exists, then we have( $\left.{ }^{(32}\right)$ :

(i) If $\gamma=0$, for $\zeta_{1}$ and $\zeta_{2}$ in $|\zeta-1|<1$, for which $0<c_{1}<\left|\zeta_{1} / \zeta_{2}\right|<c_{2}\left(c_{1}, c_{2}\right.$ consts.),

$$
\left|\frac{\omega\left(\zeta_{2}\right)}{\omega\left(\zeta_{1}\right)}\right|=\left|\frac{\zeta_{2}}{\zeta_{1}}\right|^{\theta / \pi}(1+o(1)) \quad \text { as } \zeta_{1}, \zeta_{2} \rightarrow 0,
$$

uniformly in the circle $|\zeta-1|<1$.

(ii) As $\zeta \rightarrow 0$ in $|\zeta-1|<1$ in any way at all,

$$
\frac{\log |\omega(\zeta)|}{\log |\zeta|} \rightarrow \frac{\theta}{\pi} \cos ^{2} \gamma, \quad \frac{\log \omega(\zeta)}{\log \zeta} \rightarrow \frac{\theta}{\pi} e^{i \gamma} \cos \gamma .
$$

(iii) $A s \zeta \rightarrow 0$ in any angle $|\arg \zeta| \leqq \alpha<\pi / 2\left({ }^{33}\right)$,

$$
\frac{\log \left|\omega^{\prime}(\zeta)\right|}{\log |\zeta|} \rightarrow \frac{\theta}{\pi} \cos ^{2} \gamma-1
$$

We now apply these results to various special cases.

20. Boundary "elements" with bounded argument oscillation. Let $\Gamma$ be a curve as described in $\S 19$ (a) for which $\Phi_{+}(\rho)$ and $\Phi_{-}(\rho)$ are bounded for $0<\rho \leqq a$. Then, necessarily, $\gamma=0\left({ }^{34}\right)$, and the results of $\$ 19$ hold with $\gamma$ replaced by 0 . We consider the case where

$$
\lim _{\rho \rightarrow 0} \Phi_{+}(\rho)=\phi_{+} \text {and } \lim _{\rho \rightarrow 0} \Phi_{-}(\rho)=\phi_{-} \text {exist. }
$$

(a) Corners. If $\theta \equiv \phi_{-}-\phi_{+}>0$, then $\Gamma$ has an $L$-corner of measure $\theta$ at $\omega=0$ (see $\$ 2$ ). In this case parts (ii), (iii) and (vi) of Theorem XI (A) are known, (ii) has been proved by Ostrowski, even under the weaker hypothesis that $\Gamma$ has a corner at $\omega=0$ (and not necessarily an $L$-corner), and (iii) and (vi) express the fact that the map is quasi-conformal at $\omega=0$. Furthermore, the results of the corollary for this case as well as those for $\theta=0$ are due to Ostrowski (see $\left.\left({ }^{27}\right)\right)$.

Formulas (19.3) and (19.5) give expressions for the order of magnitude of $|\zeta(\omega)|$. One may ask here, when, in particular, $\zeta(\omega) \sim c \omega^{\pi / \theta}$ as $\omega \rightarrow 0$ in $R+\Gamma$ $(c=$ constant $\neq 0)$. This question has received much attention in the recent lit-

(32) For parts (i) and (ii) see Corollary 2 of Theorem X $\left(\$ 18,\left(18.7^{*}\right)\right.$ and $\left.\left(18.8^{*}\right)\right)$.

(33) The relation (19.7) follows from Theorem XI (A), (ii) and (19.6) if one observes that

as $\zeta \rightarrow 0$ in $|\arg \zeta| \leqq \alpha<\pi / 2$.

$$
\log \left|\omega^{\prime}(\zeta)\right|=\log |\omega(\zeta)|-\log |\zeta|+\log \left[\frac{\Theta(\rho)}{\pi} \cos \gamma\right]+o(1)
$$

(34) If $\gamma$ were not equal to 0 , we would have since $\left(d \Phi_{+} / d \rho\right) \rho \rightarrow \tan \gamma,\left(\rho_{1}<\rho_{2}\right)$,

$$
\left|\Phi_{+}\left(\rho_{2}\right)-\Phi_{+}\left(\rho_{1}\right)\right|=\left|\int_{\rho_{1}}^{\rho_{2}} \frac{d \Phi_{+}}{d \rho} d \rho\right| \geqq \frac{|\tan \gamma|}{2} \int_{\rho_{1}}^{\rho_{2}} \frac{d r}{r}
$$

for all sufficiently small $\rho_{1}$ and $\rho_{2}$. Keeping $\rho_{2}$ fixed and letting $\rho_{1} \rightarrow 0$, we would find that $\Phi_{+}(\rho)$ is not bounded. 
erature, especially for the case $\theta=\pi$ where this means that $\zeta(\omega)$ possesses a nonvanishing derivative at $\omega=0$. Since by (19.1) $\lim _{\omega \rightarrow 0} \arg \left(\zeta(\omega) / \omega^{\pi / \theta}\right)$ exists, it is sufficient to consider $\lim _{\omega \rightarrow 0}\left|\zeta(\omega) / \omega^{\pi / \theta}\right|$. The remark of $\S 19$ (c) yields the following result which does not even presuppose any assumption regarding the existence of $\lim _{\rho \rightarrow 0} \theta(\rho)$ or that of the limits (20.1).

TheOREM XII. If $\Gamma$ is a curve as described in $\$ 19$ (a) and if the integrals (19.4) converge, then a necessary and sufficient condition that, for some positive $\theta$, $\lim _{\omega \rightarrow 0}\left|\zeta(\omega) / \omega^{\pi / \theta}\right|$ exist for unrestricted approach and be different from 0 is that

$$
\int_{0}^{a} \frac{\theta-\Theta(\rho)}{\rho \Theta(\rho)} d \rho \text { converge. }
$$

For, we have, uniformly in $R$,

$$
\begin{aligned}
\left|\frac{\zeta(\omega)}{\omega^{\pi / \theta}}\right| & =c^{\prime} \exp \left\{-\pi \int_{\rho}^{a} \frac{d r}{r \Theta(r)}+\frac{\pi}{\theta} \log \frac{a}{\rho}-\frac{\pi}{\theta} \log a+o(1)\right\} \\
& =c^{\prime \prime} \exp \left\{-\pi \int_{\rho}^{a} \frac{\theta-\Theta(r)}{r \theta \Theta(r)} d r+o(1)\right\}
\end{aligned}
$$

as $|\omega|=\rho \rightarrow 0$ ( $c^{\prime \prime}$ is a constant different from 0$)$.

Combining this result with (19.1) we find:

Theorem XIII. If $\Gamma$ is a curve as described in $\$ 19$ (a) and if the integrals (19.4) converge, then a necessary and sufficient condition in order that for some $\theta>0, \lim _{\omega \rightarrow 0}\left(\zeta(\omega) / \omega^{\pi / \theta}\right)$ exist for unrestricted approach and be different from 0 is that the conditions (20.1) and (20.2) be satisfied and that $\phi_{-}-\phi_{+}=\theta$.

The sufficiency of the conditions stated is clear. That (20.2) is necessary follows from Theorem XII. That (20.1) and the relation $\phi_{-}-\phi_{+}=\theta$ are necessary is immediately seen if we observe that $\arg \left\{\zeta /[\omega(\zeta)]^{\pi / \theta}\right\}$ approaches a limit as $\zeta \rightarrow 0$ in $|\zeta-1| \leqq 1$, and then let $\zeta \rightarrow 0$, first along the upper and then along the lower semi-circle of $|\zeta-1|=1$.

For $\theta=\pi$ this theorem gives a criterion for the existence of the derivative of $\zeta(\omega)$ at a boundary point. Several criteria (sufficient conditions) for the existence of the angular derivative (i.e., $\lim _{\zeta \rightarrow 0}(\omega(\zeta) / \zeta)$ in any fixed angle $|\arg \zeta| \leqq \alpha<\pi / 2)$ are known which apply to even more general types of regions than those bounded by Jordan curves. (For sufficiently smooth boundaries the existence of the derivatives for unrestricted approach can be inferred from that of the angular derivative.) The sharpest of these criteria to date is due to Ahlfors $\left({ }^{35}\right)$. Since our theorem refers to a smaller class of regions it obviously does not contain that of Ahlfors $\left({ }^{36}\right)$. On the other hand, the follow-

(35) Ahlfors $[1$, p. 36].

${ }^{\left({ }^{36}\right)}$ However, by use of Theorem XII (B) and a modification of Ahlfors' proof of his criterion, one may obtain a sharper criterion for the existence of the angular derivative. 
ing example shows that it also is not contained in Ahlfors' result: Let $\Gamma$ be a closed Jordan curve through $\omega=0$ and let, in a neighborhood of $\omega=0$ $(0 \leqq \rho \leqq a), \Gamma$ consist of the two branches $\Gamma_{+}$and $\Gamma_{-}$represented by

$\phi=\Phi_{+}(\rho) \equiv-\frac{\pi}{2}+\delta_{1}(\rho), \phi=\Phi_{-}(\rho) \equiv \frac{\pi}{2}+\delta_{2}(\rho), \delta_{i}(\rho)>0, \lim _{\rho \rightarrow 0} \delta_{i}(\rho)=0$,

where $\int_{0}^{a}\left[\delta_{i}(\rho) / \rho\right] d \rho$ is divergent $(i=1,2), \int_{0}^{a}\left\{\left|\delta_{1}(\rho)-\delta_{2}(\rho)\right| / \rho\right\} d \rho$ convergent, $\rho \delta_{i}^{\prime}(\rho)$ continuous, $\rho\left(\delta_{i}^{\prime}(\rho)\right)^{2}$ integrable, $0 \leqq \rho \leqq a$. It is easily seen that the hypotheses of Theorem XIII are satisfied and that therefore $\lim _{\omega \rightarrow 0}[\zeta(\omega) / \omega]$ exists and does not equal 0. However, one of the conditions of Ahlfors' criterion is not satisfied. Suppose $\Gamma$ is mapped by the function $w=\log (1 / \omega)$ onto a strip. The images $C_{+}$and $C_{-}$of $\Gamma_{+}$and $\Gamma_{-}$are represented by the equations

$$
v=\phi_{+}(u) \equiv \frac{\pi}{2}-\delta_{1}\left(e^{-u}\right), \quad v=\phi_{-}(u) \equiv-\frac{\pi}{2}-\delta_{2}\left(e^{-u}\right),
$$

respectively. Then (in Ahlfors' notation [1, p. 36]) the series $\sum_{\nu=1}^{\infty} m_{\nu}$ does not converge. For, if $\nu k \leqq u \leqq(\nu+1) k$ are the intervals for which the $m_{\nu}$ are formed, then

$$
k \sum_{\nu=1}^{n} m_{\nu} \geqq \sum_{k=1}^{n} \int_{\nu k}^{(\nu+1) k} \delta_{1}\left(e^{-u}\right) d u=\int_{k}^{(n+1) k} \delta_{1}\left(e^{-u}\right) d u \rightarrow+\infty
$$

as $n \rightarrow \infty$. However, the convergence of this series is one of the conditions of his criterion.

A corollary of Theorem XIII is the following

Theorem XIV. Let $\Gamma$ be a closed Jordan curve through $\omega=0$ which has an $L$-corner of measure $\theta$ at $\omega=0$, formed by the two branches $\Gamma_{+}$and $\Gamma_{-}$. Suppose that the angles of inclination of the tangents to $\Gamma_{+}$and $\Gamma_{-}$, considered as functions of $\rho$, are of bounded variation in a neighborhood of $\omega=0,0 \leqq \rho \leqq a$. Let $\zeta(\omega)$ be defined as in $\$ 19$ (a). Then, a necessary and sufficient condition that $\lim _{\omega \rightarrow 0}\left(\zeta(\omega) / \omega^{\pi / \theta}\right)$ exist and be different from zero is that the integral (20.2) converge. $\left(\Phi_{+}(\rho), \Phi_{-}(\rho)\right.$ and $\Theta(\rho)$ in (20.2) are defined as in $\$ 19$ (a).)

Proof. We represent $\Gamma_{+}$and $\Gamma_{-}$in the form $\phi=\Phi_{+}(\rho)$ and $\phi=\Phi_{-}(\rho)$ respectively, $0 \leqq \rho \leqq a$, and show first that the integrals (19.4) converge. Let $\tau_{+}(\rho)$ denote the angle of inclination of the tangent to $\Gamma_{+}$at $\omega=\rho e^{t \Phi_{+}(\rho)}$ (if the tangent exists), $\tau_{+}(\rho)$ being so chosen that $\lim _{\rho \rightarrow 0} \tau_{+}(\rho)=\phi_{+}$(see (20.1)). As is well known,

$$
\rho \frac{d \Phi_{+}(\rho)}{d \rho}=\tan \left[\tau_{+}(\rho)-\Phi_{+}(\rho)\right]
$$


To prove the convergence of the first integral in (19.4) it is sufficient to show that

$$
\int_{0}^{a}\left[\tau_{+}(\rho)-\Phi_{+}(\rho)\right] \frac{d \Phi_{+}(\rho)}{d \rho} d \rho \text { converges. }
$$

Integration by parts gives $(0 \leqq \epsilon<a)$

$$
\begin{aligned}
\int_{e}^{a}\left[\tau_{+}-\Phi_{+}\right] \frac{d \Phi_{+}}{d \rho} d \rho= & -\int_{\rho=\epsilon}^{a}\left(\Phi_{+}(\rho)-\phi_{+}\right) d\left[\tau_{+}(\rho)-\Phi_{+}(\rho)\right] \\
& +\left.\left(\Phi_{+}(\rho)-\phi_{+}\right)\left(\tau_{+}(\rho)-\Phi_{+}(\rho)\right)\right|_{\epsilon} ^{a} \\
= & -\int_{\rho=\epsilon}^{a}\left(\Phi_{+}(\rho)-\phi_{+}\right) d \tau_{+}(\rho) \\
& +\int_{\rho=\epsilon}^{a}\left(\Phi_{+}(\rho)-\phi_{+}\right) d\left[\Phi_{+}(\rho)-\phi_{+}\right]+M(\epsilon, a),
\end{aligned}
$$

where $M(\epsilon, a)$ is continuous at $\epsilon=0$. Since $\tau_{+}(\rho)$ is of bounded variation and $\Phi_{+}(\rho)$ is continuous for $0 \leqq \rho \leqq a$, the first of the last two integrals converges as $\epsilon \rightarrow 0$. The second of these integrals has the value $\left[\frac{1}{2}\left(\Phi_{+}(\rho)-\phi_{+}\right)^{2}\right]_{e}^{a}$ and it approaches, therefore, a finite limit as $\epsilon \rightarrow 0$. This proves (20.3).

Similarly it is shown that the second integral in (19.4) converges and Theorem XIV follows now from Theorem XIII.

(b) Cusps. Suppose now that $\Gamma$ has an $L$-cusp at $\omega=0$. Our main results here are the formulas (19.3), with $\gamma=0$, and (19.5) which give asymptotic expressions for $|\zeta(\omega)|$. Parts (iii) and (vi) of Theorem XI (A) take the place of the quasi-conformality in the case of a corner. Part (ii) of this theorem (with $\gamma=0$ ) is an extension of Ostrowski's result which states that under the weaker assumption that $\Gamma$ has a cusp at $\omega=0$ (and not necessarily an $L$-cusp), $\lim _{\zeta \rightarrow 0}\left[\omega^{\prime}(\zeta) /(\omega(\zeta) / \zeta)\right]=0$, in any fixed angle $|\arg \zeta| \leqq \beta<\pi / 2$ (cf. the remark to Theorem X, §16). Similarly, part (i) of Theorem XI (A) can be considered as a sharper form of (19.6), since it gives the order of magnitude of $\log |\zeta(\omega)|$ while (19.6) merely states that $(\log |\zeta(\omega)| / \log |\omega|) \rightarrow+\infty$ as $\omega \rightarrow 0$. The results of the corollary are due to Ostrowski (see $\left.\left({ }^{27}\right)\right)$.

EXAMPLE. It might be of some interest to apply our results to a cusp formed by two arcs $\Gamma_{+}$and $\Gamma_{-}$which have "finite order of contact." $\Gamma_{+}$and $\Gamma_{-}$ are represented in Cartesian coordinates $(\xi, \eta)$ by the equations

$$
\eta=[a+\epsilon(\xi)] \xi^{n}, \quad \eta=[b+\delta(\xi)] \xi^{m}, \quad 0 \leqq \xi \leqq \xi_{0} .
$$

Here $a, b, n, m$ are any real numbers, $n \geqq m>1$; if $n>m$ then $b>0$, if $n=m$, $b>a ; \epsilon(\xi), \delta(\xi)$ have continuous first derivatives, $0 \leqq \xi \leqq \xi_{0}$ and approach 0 with $\xi$. It is clear that $\Gamma_{+}$and $\Gamma_{-}$form an $L$-cusp so that Theorem XI (A) holds. We examine, therefore, the possibility of applying part (B). 
We introduce polar coordinates about $\omega=0$. Using $\rho$ as parameter and writing $\phi=\Phi_{+}(\rho)$ on $\Gamma_{+}$and $\phi=\Phi_{-}(\rho)$ on $\Gamma_{-}$we obtain from (20.4), $\rho>0$, $(20.5) \rho \sin \Phi_{+}(\rho)=[a+\epsilon(\xi)] \rho^{n} \cos ^{n} \Phi_{+}(\rho), \rho \sin \Phi_{-}(\rho)=[b+\delta(\xi)] \rho^{m} \cos ^{m} \Phi_{-}(\rho)$.

\section{Hence}

$\sin \left[\Phi_{-}(\rho)-\Phi_{+}(\rho)\right]=\rho^{m-1}\left\{(b+\delta) \cos ^{m} \Phi_{-} \cos \Phi_{+}-(a+\epsilon) \rho^{n-m} \cos ^{n} \Phi_{+} \cos \Phi_{-}\right\}$.

Thus we find

$$
\Theta(\rho)=\Phi_{-}-\Phi_{+} \sim b \rho^{m-1} \quad \text { if } \quad n>m ; \quad \Theta(\rho) \sim(b-a) \rho^{m-1} \quad \text { if } n=m,
$$

as $\rho \rightarrow 0$. Furthermore, differentiation of the first relation of (20.5) with respect to $\rho$ gives

$$
\cos \Phi_{+}(\rho) \frac{d \Phi_{+}}{d \rho}=(a+\epsilon)(n-1) \rho^{n-2}\{1+o(1)\} \quad \text { as } \rho \rightarrow 0,
$$

so that

$$
\frac{\rho}{\Theta(\rho)}\left(\frac{d \Phi_{+}}{d \rho}\right)^{2}=O\left(\rho^{n-2}\right)
$$

and similarly

$$
\frac{\rho}{\Theta(\rho)}\left(\frac{d \Phi_{-}}{d \rho}\right)^{2}=O\left(-\rho^{m-2}\right) .
$$

Hence the conditions (19.4) of the remark of $\$ 19$ (c) are satisfied and therefore (19.5) holds.

21. Boundary "elements" with unbounded argument oscillation. Suppose that $\Gamma$ is a curve as described in $\$ 19$ (a) and that $\lim _{\rho \rightarrow 0} \Phi_{+}(\rho)$ and $\lim _{\rho \rightarrow 0} \Phi_{-}(\rho)$ are both $+\infty$ (or $-\infty$ ). In this case $\Gamma_{+}$and $\Gamma_{-}$are two "concurrent" spirals having $\omega=0$ as an asymptotic point. Our results of $\$ 19$ described the behavior of $\zeta(\omega)$ and $\zeta^{\prime}(\omega)$ as $\omega$ approaches the asymptotic point.

However, our methods still apply to a case not included in $\$ 19$, in which $\rho\left(d \Phi_{+} / d \rho\right)$ and $\rho\left(d \Phi_{-} / d \rho\right)$ both approach $+\infty$ (or $\left.-\infty\right)$. This case is contained in the following more general configuration.

Let $R$ be a simply-connected (single-sheeted) region whose boundary consists of the spirals

$$
\Gamma_{+}: \quad \rho=\rho_{+}(\phi), \quad \Gamma_{-}: \quad \rho=\rho_{-}(\phi), \quad \phi_{0} \leqq \phi<\infty,
$$

and of a Jordan arc connecting the end points $\left(\phi_{0}, \rho_{+}\left(\phi_{0}\right)\right)$ of $\Gamma_{+}$and $\left(\phi_{0}, \rho_{-}\left(\phi_{0}\right)\right)$ of $\Gamma_{-}$. It is assumed that $\rho_{+}(\phi)$ and $\rho_{-}(\phi)$ are positive and absolutely continuous in any interval $\phi_{0} \leqq \phi \leqq \phi_{1}<\infty$, and that $\left(1 / \rho_{+}(\phi)\right) d \rho_{+} / d \phi$ and $\left(1 / \rho_{-}(\phi)\right) d \rho_{-} / d \rho$ which exist for almost all $\phi \geqq \phi_{0}$, both approach the limit $-\tan \gamma,|\gamma|<\pi / 2$ as $\phi \rightarrow \infty$. The region

$$
\phi_{0}<\phi<\infty, \quad \rho_{+}(\phi)<\rho<\rho_{-}(\phi)
$$


is contained in $R$. We set

$$
\Theta(\phi) \equiv \log \frac{1}{\rho_{+}(\phi)}-\log \frac{1}{\rho_{-}(\phi)}, \quad \Psi(\rho) \equiv \frac{1}{2}\left[\log \frac{1}{\rho_{+}(\phi)}+\log \frac{1}{\rho_{-}(\phi)}\right] .
$$

There exists a function $\zeta(\omega)$ which maps $R$ conformally onto the circle $|\zeta-1|<1$ in such a manner that $\lim \zeta(\omega)=0$ as arg $\omega \rightarrow+\infty, \omega$ being in the region (21.1). The inverse function of $\zeta(\omega)$ will again be denoted by $\omega(\zeta)$.

The function $w=u+i v=i \log (1 / \omega)=i \log (1 / \rho)+\phi$ maps $R$ onto an $L$-strip $S$ in the $w$-plane with the boundary inclination $\gamma$ at $u=+\infty$. We map the circle $|\zeta-1|<1$ onto the strip $|y|<\pi / 2$ by means of the function $z=\log \{(2-\zeta) / \zeta\} \quad(z=0$, when $\zeta=1)$. In this way we have again reduced our problem to that of a strip. We can carry over all our theorems on $L$-strips thus obtaining results on $\zeta(\omega)$ and $\omega(\zeta)$ as arg $\omega \rightarrow+\infty$ for $\omega$ in (21.1) and $\zeta \rightarrow 0$ in $|\zeta-1|<1$.

To apply Theorem IX and to obtain the analogue of Theorem XI (B) we assume here that the integrals

$$
\int_{\phi=\phi_{0}}^{\infty}\left|d\left(\frac{\rho_{+}^{\prime}(\phi)}{\rho_{+}(\phi)}\right)\right|, \quad \int_{\phi=\phi_{0}}^{\infty}\left|d\left(\frac{\rho_{-}^{\prime}(\phi)}{\rho_{-}(\phi)}\right)\right|, \quad \int_{\phi_{0}}^{\infty} \frac{\Theta^{\prime 2}(\phi)}{\Theta(\phi)} d \phi
$$

converge. There is no difficulty in actually writing out the results obtained for this case.

\section{BibLIOGRAPHY}

L. AHLFORS

1. Untersuchungen zur Theorie der konformen Abbildung und der ganzen Funktionen, Acta Societatis Scientiarum Fennicae, (n. s.), A, vol. 1 (1930).

C. CARATh ÉDORY

1. Elementarer Beweis für den Fundamentalsatz der konformen Abbildung, Schwarz-Festschrift, 1914, pp. 19-41.

2. Conformal Representation, Cambridge Tracts, 1932.

E. T. Copson

1. Introduction to the Theory of Functions of a Complex Variable, Oxford University Press, 1935.

W. Gross

1. Zum Verhalten analytischer Funktionen in der Umgebung singulärer Stellen, Mathematische Zeitschrift, vol. 2 (1918), pp. 241-294.

E. LINDEL ÖF

1. Sur la représentation conforme d'une aire simplement connexe sur l'aire d'un circle, Compte Rendu du Quatrième Congrès des Mathématiciens Scandinaves, Stockholm, (1916), pp. 59-90.

R. NevanlinNa

1. Eindeutige analytische Funktionen, Berlin, 1936.

A. Ostrowski

1. Über den Habitus der konformen Abbildung am Rande des Abbildungsbereiches, Acta Mathematica, vol. 64 (1934), pp. 81-185.

2. Zur Randverzerrung bei konformer Abbildung, Prace Matematiczno Fiziczne, vol. 44 (1937), pp. 371-471. 
T. RADó

1. Sur la représentation conforme de domaines variables, Acta Litterarum ac Scientiarum Universitatis Francisco Josephinae, vol. 1 (1923), pp. 180-186.

S. E. WARSChaWSKI

1. Zur Randverzerrung bei konformer Abbildung, Compositio Mathematica, vol. 1 (1935), pp. 314-343.

2. On the preservation of angles at a boundary point in conformal mapping, Bulletin of the American Mathematical Society, vol. 42 (1936), pp. 674-680.

\section{J. WOLFF}

1. Sur la représentation conforme des bandes, Compositio Mathematica, vol. 1 (1934), pp. 217-222.

2. Sur la conservation des angles dans la représentation conforme d'un domaine au voisinage d'un point frontière, Comptes Rendus de l'Académie des Sciences, Paris, vol. 200 (1935), pp. 42-43.

3. Démonstration d'un théorème sur la conservation des angles dans la représentation conforme d'un domaine au voisinage d'un point frontière, Proceedings, Akademie van Wetenschappen, Amsterdam, vol. 38 (1935), pp. 46-50.

WASHINGTON UNIVERSITY, .

St. Lours, Mo. 